IZA DP No. 7583

Regression Analysis of Country Effects Using Multilevel Data: A Cautionary Tale

Mark L. Bryan

Stephen P. Jenkins

August 2013

Forschungsinstitut zur Zukunft der Arbeit Institute for the Study of Labor 


\title{
Regression Analysis of Country Effects Using Multilevel Data: A Cautionary Tale
}

\author{
Mark L. Bryan \\ ISER, University of Essex \\ Stephen P. Jenkins \\ London School of Economics, \\ ISER and IZA
}

\section{Discussion Paper No. 7583 \\ August 2013}

\author{
IZA \\ P.O. Box 7240 \\ 53072 Bonn \\ Germany \\ Phone: +49-228-3894-0 \\ Fax: +49-228-3894-180 \\ E-mail: iza@iza.org
}

Any opinions expressed here are those of the author(s) and not those of IZA. Research published in this series may include views on policy, but the institute itself takes no institutional policy positions. The IZA research network is committed to the IZA Guiding Principles of Research Integrity.

The Institute for the Study of Labor (IZA) in Bonn is a local and virtual international research center and a place of communication between science, politics and business. IZA is an independent nonprofit organization supported by Deutsche Post Foundation. The center is associated with the University of Bonn and offers a stimulating research environment through its international network, workshops and conferences, data service, project support, research visits and doctoral program. IZA engages in (i) original and internationally competitive research in all fields of labor economics, (ii) development of policy concepts, and (iii) dissemination of research results and concepts to the interested public.

IZA Discussion Papers often represent preliminary work and are circulated to encourage discussion. Citation of such a paper should account for its provisional character. A revised version may be available directly from the author. 
IZA Discussion Paper No. 7583

August 2013

\section{ABSTRACT}

\section{Regression Analysis of Country Effects Using Multilevel Data: A Cautionary Tale*}

Cross-national differences in outcomes are often analysed using regression analysis of multilevel country datasets, examples of which include the ECHP, ESS, EU-SILC, EVS, ISSP, and SHARE. We review the regression methods applicable to this data structure, pointing out problems with the assessment of country-level factors that appear not to be widely appreciated, and illustrate our arguments using Monte-Carlo simulations and analysis of women's employment probabilities and work hours using EU SILC data. With large sample sizes of individuals within each country but a small number of countries, analysts can reliably estimate individual-level effects within each country but estimates of parameters summarising country effects are likely to be unreliable. Multilevel (hierarchical) modelling methods are commonly used in this context but they are no panacea.

JEL Classification: C52, C81, O57

Keywords: multilevel modelling, cross-national comparisons, country effects

Corresponding author:

Stephen P. Jenkins

Department of Social Policy

London School of Economics and Political Science

Houghton Street

London WC2A 2AE

United Kingdom

E-mail: s.jenkins@lse.ac.uk

\footnotetext{
* This research was supported by funding from the UK Economic and Social Research Council for the Analysing Life Changes in Europe project (grant no. RES-062-23-1455). Core funding from the ESRC (grant RES-518-28-001) and the University of Essex for the Research Centre on Micro-Social Change at ISER is also acknowledged. For assistance, comments and suggestions, we thank Bjaarte Aagnes, William Buchanan, Joerg Luedicke, Henning Lohmann, Matthias Parey, Steve Pudney, Jay Verkuilen, our ALiCE project collaborators, and seminar participants at IoE, LSHTM, and Oxford.
} 


\section{Introduction}

Researchers often wish to compare and explain differences in socio-economic outcomes across countries. They aim to discover how different policy environments and institutions affect outcomes and to inform the policy debate about how to improve outcomes. Many types of empirical approach are used in cross-national comparative work. On the one hand, qualitative methods include analysis of interviews with key informants and examination of documents summarising national laws and institutions. On the other hand, quantitative methods are based on survey or register data or other administrative sources (e.g. official statistics). The most popular quantitative approach is multivariate regression analysis of data from surveys or registers in multiple countries in which individual outcomes are modelled as a function of both individual-level and country-level characteristics. The properties of estimates from this approach are the subject of this paper. ${ }^{1}$ We argue that the small number of countries in most multi-country datasets severely constrains the ability of regression models, including multilevel (hierarchical) models, to provide robust conclusions about the effects of country-level characteristics on outcomes.

Multi-country datasets that are commonly-used in contemporary social science research are summarised in Table 1. Common to them is their multilevel structure: there are observations at the individual level nested within a higher level (countries), so there is a natural hierarchy within the data. (When repeated waves of the same survey are available, the second level may be the country-year, with the country itself as a third level.) The datasets listed typically contain thousands of observation at the individual level, but the number of countries is relatively small and typically around 30: see the right-hand column of Table 1. The number of countries with data useable in regression analysis is often fewer still, e.g. because of missing data for some variables.

Multi-country datasets are attractive to researchers because they offer a means of quantifying the way in which countries matter for outcomes - the extent to which differences in outcomes reflect differences in the effects of country-specific features of demographic structure, labour markets and other socio-economic institutions such as tax-benefit systems that are distinct from the differences in outcomes associated with variations in the characteristics of the individuals themselves. In other words, multi-country datasets

\footnotetext{
${ }^{1}$ Not all quantitative cross-national comparative research uses multivariate regression of multilevel data. Other methods include decomposition of measures of inequality and poverty. There is another stream of literature which uses countries as the level of observation, often using country-level panels (cf. Beck and Katz 1995).
} 
potentially provide information about ‘country effects' as well as 'individual effects', and also about interactions between them ('cross-level effects').

The popularity among quantitative sociologists of regression analysis of multilevel country data is illustrated by the articles published in the European Sociological Review between 2005 and 2012. Of the 340 articles published, we identify approximately 75 that exploit multilevel datasets with individual respondents within countries. Of course there are articles based on regression analysis of multilevel country data in other social science journals as well. (For example, there are 14 out of the 111 articles in the Journal of European Social Policy between 2005 and 2009, and 10 articles in a special issue of Political Analysis in 2005.) The various types of regression analysis that are employed in these studies are reviewed later in the paper. ${ }^{2}$ The topics addressed vary widely, reflecting survey content, ranging for example from labour force participation and wages to political and civic participation rates, and social and political attitudes.

Multilevel data sets are examples of what statisticians refer to as cluster samples: there are individual units sampled within groups or clusters. The key issue for estimation and subsequent substantive interpretation is how to model differences in outcomes within and between the clusters. There are several different approaches, but the most popular in the multilevel country case is multilevel (hierarchical) regression modelling using specialist software such as HLM or MLwiN or modules within general statistical software such as Stata or SAS. Multilevel modelling is used in 43 of the 75 articles in the European Sociological Review cited earlier (i.e. 57 per cent; or 13 per cent of all 340 articles).

In this paper, we argue that, for the multilevel country data case, there are problems when the number of countries is small - which is the usual situation (Table 1). The intuition is straightforward: in general, desirable properties of regression model parameter estimates such as consistency and efficiency are contingent on sample sizes being 'large'. In particular, a large number of groups (countries) is needed in order to estimate country effects reliably. The caveat applies both to the 'fixed' parameters associated with country-level explanatory variables (and individual-country level interactions) and to the variances of random countryspecific parameters (intercepts and slopes). It is a generic problem that affects all regression modelling approaches; using multilevel regression models is no panacea. Although software

\footnotetext{
${ }^{2}$ Between 2005 and 2012, the European Sociological Review also published 8 articles that exploit multilevel data but where the structure refers to pupils nested within schools or to respondents within geographical areas.
} 
produces estimates of individual- and country-level effects and estimates of their statistical significance, the issue is: which of these estimates can be trusted and in what circumstances?

We provide answers to this question. Drawing on literature from several social science disciplines, we aim to provide a unified treatment for quantitative social science researchers as the issues that we discuss appear to be not widely appreciated among this audience. Our exposition is intended to be accessible to applied researchers who do not have specialist statistical knowledge and so, wherever possible, we have relegated technical explanations and details to footnotes. In the next section, we review four regression modelling approaches to modelling individual and country effects from multilevel country data. We explain in more detail the issues associated with estimation of country effects in the following three sections. We begin the discussion with reference to the simplest case, a linear model in which country effects are characterised as random differences in model intercepts (section 3), and then extend the discussion to more complex models with country differences in slopes as well as intercepts (section 4), and also to non-linear models for binary outcomes (section 6). We argue that viewing estimation of individual and country effects in terms of a two-step procedure can help to clarify the sources of the problems with small sample sizes. Throughout, we refer to cross-sectional data sets; the case of multi-country panels or other forms of longitudinal dataset are not considered explicitly.

We review the literature on the performance of multilevel estimators in section 5 . Because most existing literature does not cover the data structure of interest here, we present our own Monte-Carlo simulation analysis of the properties of multilevel estimators (section 7). Unlike previous studies, we focus on data structures that are typical of cross-country research, examining estimator performance with as few as 5 groups, while maintaining a large group size (1,000 observations per group). Moreover, we go beyond the linear models that have predominated in the multilevel simulation literature, and evaluate the performance of non-linear models (logit) models that are common in applied research, and we draw out some rules of thumb. Informed by these Monte Carlo results, we compare the various estimation approaches outlined earlier using linear and non-linear models estimated on multilevel country data from EU-SILC (section 8). In the final section, we summarise our conclusions and offer advice about regression modelling of multilevel country data. 


\section{Regression analysis of multilevel country data: four approaches}

Before considering estimation issues in detail, we review four regression approaches that an analyst might use with multilevel country data. ${ }^{3}$ The discussion begins with reference to a linear model for a metric outcome variable:

$$
y_{i c}=\boldsymbol{X}_{i c} \boldsymbol{\beta}+\boldsymbol{Z}_{c} \boldsymbol{\gamma}+u_{c}+\varepsilon_{i c}, \text { with } i=1, \ldots, N_{c} ; c=1, \ldots, C .
$$

Outcome $y_{i c}$ for each person $i$ in country $c$ is assumed to depend on both observed predictors and unobserved factors. $\boldsymbol{X}_{i c}$ contains variables that summarise individual-level characteristics such as age, education or marital status; $\boldsymbol{Z}_{c}$ contains variables summarising country-level features such as socio-economic institutions or labour markets. There are also unobserved individual effects $\left(\varepsilon_{i c}\right)$ and country effects $\left(u_{c}\right)$ that are each assumed to be normally distributed and uncorrelated with $\boldsymbol{X}_{i c}$ and $\boldsymbol{Z}_{c}$. Unless stated otherwise, we have in mind a dataset with a large number of individuals for each country ( $N_{c}$ is typically in the thousands) sampled from each of a small number of countries ( $C$ is around 30 or fewer). The parameters associated with the observed predictors $\boldsymbol{\beta}$ and $\boldsymbol{\gamma}$ are sometimes called 'fixed' regression parameters in order to distinguish them from the parameters characterising the joint distribution of the 'random' terms $\varepsilon_{i c}$ and $u_{c}$, such as $\operatorname{var}\left(\varepsilon_{i c}\right)$ and $\operatorname{var}\left(u_{c}\right)$ although note that, in two of the approaches below, $u_{c}$ is also treated as a fixed parameter.

Pooling the data for all countries (and using cluster-robust standard errors)

A first approach is to simply pool the data from all of the country surveys. If one disregards the nesting of observations within countries, this approach ignores the fact that individuals within a country share unobserved characteristics ( $u_{c}$ is an omitted variable). This leads to underestimation of the standard errors of $\beta$ because the within-group (intra-class) correlation across individual units is not accounted for (Moulton 1986). Fortunately, it is straightforward to apply a 'Moulton correction' or, more commonly, to allow for more general correlation structure among individuals within countries using estimates of cluster-robust standard errors where the clusters are the countries (Angrist and Pischke 2009: 312-3). ${ }^{4}$ Another possibility

\footnotetext{
${ }^{3}$ Our discussion is limited to the classical statistical framework favoured by most applied researchers. Bayesian methods offer a potential way to address the small numbers issues, contingent on making assumptions about 'prior distributions' of parameters including regarding country effects. See inter alia Browne and Draper (2006 and Gelman (2006). Bayesian methods are not yet widely used by social science researchers. One exception is the application by Kedar (2005) in which the number of second level units is 14 .

${ }^{4}$ In Stata, one would use the regression command option cluster(country_identifier).
} 
is to derive standard errors with block-bootstrap techniques (Angrist and Pischke 2009: 315; Cameron, Gelbach, and Miller 2008). Although cluster-robust standard errors are easy to derive nowadays, reliance on them is a conservative strategy because the within-country correlation is controlled for but not explicitly modelled. There are no estimates of parameters describing the distributions of the unobserved factors.

The other three approaches account for the hierarchical nature of the data explicitly.

\section{Separate models for each country}

Researchers can fit a separate model to each country's dataset. In this case, any country effect $\left(u_{c}\right)$ is absorbed into, and cannot be identified separately from, the intercept term in each country's regression model (and so is a fixed parameter included as an element of $\boldsymbol{\beta}$ ). This approach has the advantage of allowing the estimates of the coefficients on individual-level characteristics (the elements of $\boldsymbol{\beta}$ other than the intercept) to differ across countries. In addition, no restrictions are placed on the variance of the individual-specific error terms for each country.

\section{Country fixed effects (FE) models}

In a fixed effects (FE) approach, the data from the country surveys are pooled but the model specification includes distinct country intercepts (estimated as the coefficients on country binary indicator variables). Again, the country effects are treated as fixed parameters rather than random terms, with each country intercept representing the effects of unobserved factors that are shared within each country. In the simplest case, the individual effects (the nonintercept elements of $\boldsymbol{\beta}$ ) are constrained to be equal across countries, but they can be allowed to differ between countries by interacting subsets of individual-level characteristics with the country indicator variables. Estimates from a model that includes a full set of interactions between individual characteristics and the country dummies are not equivalent to the estimates derived from distinct country regressions because the residual error variance is constrained to be the same across countries in the former case but not in the latter. 


\section{Country random effects $(R E)$ models}

The random effects (RE) approach also pools the data and allows for country effects. However, rather than treating these as distinct values each of which can be estimated, they are modelled as random draws from a distribution (usually normal) with mean zero and variance which is estimated. Although this approach is termed 'random effects', the parameters $\boldsymbol{\beta}$ and $\boldsymbol{\gamma}$ remain fixed in the simple case; in a more complicated RE model they may also be allowed to vary randomly (see section 4). One of the attractions of the RE approach is that country-level regressors can also be used as model predictors (see below). By contrast, in the FE approach, country differences are fully characterised by the country indicator variables.

The RE model is the prototypical multilevel (hierarchical) model with random intercepts. A key parameter is the intra-class correlation $\rho=\sigma_{u}{ }^{2} /\left(\sigma_{\varepsilon}{ }^{2}+\sigma_{u}{ }^{2}\right)$, where $\sigma_{\varepsilon}{ }^{2}$ and $\sigma_{u}{ }^{2}$ are the variances of the individual and country random effects respectively. (Individual random effects $\left(\varepsilon_{i c}\right)$ and country random effects $\left(u_{c}\right)$ are assumed to be uncorrelated with $\boldsymbol{X}_{i c}$ and $\boldsymbol{Z}_{c}$ and with each other.) The intra-class correlation summarises the extent to which unobserved factors within each country are shared by individuals. It tends to zero as $\sigma_{u}{ }^{2} \rightarrow 0$. Assuming that the correlation structure of the random effects has a particular form leads to more efficient estimates of the individual-level effects represented by $\boldsymbol{\beta}$, i.e. estimates with standard errors smaller than the cluster-robust ones. (Of course, the efficiency gain is conditional on the model being correct.) Estimation methods for this type of model include generalised least squares (GLS), full maximum likelihood (FML) and restricted maximum likelihood (REML): see Hox (2010) for a comparative discussion. All three types of estimator deliver consistent parameter estimates, i.e. they converge to their true values in sufficiently large samples (many countries and many individual units per country). The estimate of every parameter is asymptotically normally distributed, so standard methods can be used for hypothesis testing and confidence intervals, again conditional on the large sample condition being satisfied. As discussed in more detail below, some methods may also be available for inference in small samples (Kenward and Roger 1997) or if the random effects are not normally distributed (Carpenter et al. 2003). 
Which approach should an analyst use?

Because the four approaches differ in fundamental ways, one cannot straightforwardly recommend one approach over another. Nevertheless, one can distinguish some broad considerations. First, there are distinctions between the FE and RE approaches that go beyond questions of statistical specification. The two models are conceptually different and this has implications for the inferences that can be drawn from them, especially when using multilevel country data for only a few countries. In the FE approach, the emphasis is on the uniqueness of each country: the country effect (e.g. national culture or institutions) is treated as a characteristic that cannot be transferred to another national context. It is an effect that needs to be included as a control in the model, but each country's estimate has no particular meaning regarding another country. That is, estimates from an FE approach (intercepts and coefficients) relate specifically to the set of countries included in the sample and cannot be generalised out of sample. As an example, FE estimates from a dataset including respondents from the original 15 European Union member states could not be applied to describe outcomes for the 12 new member states with their very different institutions and history. (The post-war experience of Slovenia is very different to that of France, for instance.)

Another consequence of the FE approach is that country-level variables cannot be included as additional predictors (e.g. parental leave laws affecting couples’ division of childcare time) because the country intercepts already fully encapsulate cross-country differences (Snijders and Bosker 1999). The limited conclusions in this case are a consequence of the agnostic view about the nature of country effects. To say more, additional assumptions have to be made.

The emphasis in RE models is very different: the set of countries included in the analysis is modelled as a sample from a larger population of countries defined in terms of observed country characteristics. Any remaining unobserved country effects are treated as being generated by some common mechanism and so are 'exchangeable' between countries (Snijders and Bosker 1999). The regression intercept is a population average (a common European intercept in the EU example) and deviations from this average are assumed to be uncorrelated with country-level variables included in the model. With these assumptions, the $\mathrm{RE}$ results can be generalised to other countries with different policies and institutions. For example, estimate of the effects of parental leave legislation on childcare time based on the old EU countries may be applied to possible legislative changes in the new member states. 
The second consideration relates to statistical performance. Provided there is no correlation between the unobserved group-specific effect and the regressors, FE and RE both deliver consistent estimates of $\boldsymbol{\beta}$ but the RE approach is more efficient because it 'borrows strength' from between-group variation (FE uses only within-group variation). However, in practice, the difference between the RE and FE estimates is likely to be negligible when using cross-country data that contain many more observations within countries than there are countries (large $N_{C}$, small $C$ ). This is because, with large $N_{C}$, almost all the variation used in RE estimation is from within, rather than between, countries. ${ }^{5}$ Thus the efficiency loss from using FE rather than RE (to estimate $\boldsymbol{\beta}$ ) may be negligible: with only a few countries there is little potential to 'borrow strength’ across them.

Because the differences between the FE and RE estimates of $\boldsymbol{\beta}$ are likely to be minor when using cross-county data, the choice between the two approaches (and the other methods) may largely depend on which parameters are the substantive focus of interest. Analysts primarily interested in the individual effects associated with observed predictors $(\boldsymbol{\beta})$ may favour the FE approach or separate equations. On the other hand the RE approach is the natural choice if the focus is on the effects $(\gamma)$ of country-level predictors or the variance component structure. To some extent this aspect is related to disciplinary conventions. Economists have conventionally avoided RE approaches, preferring to use one of the other three approaches. Other social scientists, including quantitative sociologists, have tended to favour the multilevel or hierarchical RE modelling approach. Henceforth we also focus the discussion on a RE framework, given our interest in the effects of both individual- and country-level predictors (and random country-specific parameters).

\section{Regression analysis of multilevel country data: a two-step approach}

It is instructive to consider a fifth approach in which estimation of the model specified in (1) is undertaken in two steps. This perspective has several advantages: first, it highlights the sources of variation in the data and illustrates why a small number of countries affects the reliability of estimates; second, the estimates are unbiased (with correct standard errors) and so can be used as a benchmark for the other methods; and third, the two-step method leads

\footnotetext{
${ }^{5}$ For example GLS estimation of (1) weights between- and within-country variation as a function of $\sigma_{\varepsilon}{ }^{2} /\left(\sigma_{\varepsilon}{ }^{2}+\right.$ $N_{C} \sigma_{u}{ }^{2}$ ). As $N_{C}$ becomes large, the fraction of between-country variation used tends to zero and GLS converges to the within-country (FE) estimator.
} 
naturally to an alternative (or complementary) graphical approach that provides a nonstatistical view of country-level variation.

The two-step approach consists of one regression at the individual level and another regression at the country level. Two-step estimation of hierarchical structures dates back to at least Hanushek (1974) and Saxonhouse (1976) among economists, but the method appears to have been periodically rediscovered. Borjas and Sueyoshi (1994) presented a two-step estimator for the probit model, and other proponents include Card (1995), and Jusko and Shively (2005) and other papers in a special issue of Political Analysis (Kedar and Shively 2005). Donald and Lang (2007) discuss the statistical properties of the two-step estimator (compared to GLS) in detail. For textbook discussion, see Wooldridge (2010: chapter 20).

In the first (within country) step, we estimate

$$
y_{i c}=\boldsymbol{X}_{i c} \boldsymbol{\beta}+v_{c}+\varepsilon_{i c}, \quad \text { with } i=1, \ldots, N_{c} ; c=1, \ldots, C
$$

where $v_{c}$ is a fixed effect for country $c$ that combines both observed and unobserved country characteristics, i.e. $v_{c}=Z_{c} \gamma+u_{c}$. In practice, this is fitted either by letting $v_{c}$ be a countryspecific binary indicator variable in an OLS regression (cf. approach 2 above) or by using the within-group estimator with the country as the group (for textbook discussion of both estimation approaches, and their equivalence, see Hsiao 2003: section 3.2). In the second step we estimate

$$
\hat{v}_{c}=\alpha+Z_{c} \gamma+\eta_{c}, \quad \text { with } c=1, \ldots, C \text {. }
$$

where $\hat{v}_{c}$ is an estimate of the country-specific fixed effect and $\eta_{c}$ is a residual error term. Depending on the first-step estimation method, $\hat{v}_{c}$ is either the coefficient on the country indicator variable or is derived from the estimates as $\hat{v}_{c}=\bar{y}_{c}-\bar{X}_{c} \hat{\boldsymbol{\beta}}$, where the bars over variables denote means taken over all individuals within a country. With large $N_{c}$, the second step can be estimated by applying OLS to the $C$ country-level observations (Donald and Lang 2007, Wooldridge 2010: 891-892). ${ }^{6}$

\footnotetext{
${ }^{6}$ The country-level error, $\eta_{c}$, in (3) can be written $\eta_{c}=u_{c}+\bar{\varepsilon}_{c}+\bar{X}_{c}(\beta-\hat{\beta})$. With large $N_{c}, \bar{\varepsilon}_{c}$ can be ignored because its variance $\left(=\sigma_{\varepsilon}^{2} / N_{c}\right)$ will be negligible compared to that of $u_{c}$, the unobserved country-specific effect. The term $\bar{X}_{c}(\beta-\hat{\beta})$, the sampling error of the estimated country effects, is heteroscedastistic, but with large $N_{c}$ it is also small. As $N_{C} \rightarrow \infty$ the equation error then converges to $u_{c}$, which by assumption is homoscedastistic and normal (Donald and Lang 2007: 225; Wooldridge 2010: 892). Therefore step 2 can be estimated efficiently using OLS, with hypothesis testing of $\gamma$ based on the $t$-distribution (with $C-k-1$ degrees of freedom, where $k$ is the number of $\boldsymbol{Z}_{c}$ variables). In the more general case of a heteroscedastistic error $\eta_{c}$ at step 2, GLS would be the efficient estimator. Borjas and Sueyoshi (1994), Hanushek (1974) and Donald and Lang (2007) provide alternative calculations of the weighting matrix for feasible GLS. However, feasible GLS estimates are only consistent (and distributed normally) for large $C$ (because estimates of the weighting matrix are 'unreliable' with
} 
Under the assumptions of the basic model (Section 2) and with large $N_{c}$, the estimates of both $\gamma$ and $\boldsymbol{\beta}$ are unbiased and have the correct standard errors. In addition the $t$ statistics and $p$-values reported as standard by software packages will lead to reliable hypothesis tests. Moreover, OLS at step 2 provides an unbiased estimate of the variance of the country effects, $\sigma_{u}{ }^{2}$. These properties apply even if there are few countries (small $C$ ), and so the two-step method can be seen as a useful benchmark for comparison with the other approaches. Closer consideration of the two-step method also highlights a number of issues that apply more generally to estimation using clustered data with few groups.

First, step 1 uses only within-country variation to estimate the individual-level parameters, $\boldsymbol{\beta}$, in contrast to the RE (and pooled) approach, which also uses between-country variation. The ability to ‘borrow strength' from across groups (countries) is often cited as an advantage (increasing efficiency) of the RE approach in estimating $\beta$. But, as noted by Aachen (2005), with only a small number of groups but large numbers of individual units within groups, there is much less need (and less potential) to borrow strength across groups. In this case the RE approach uses mainly within-country variation and the resulting $\boldsymbol{\beta}$ estimates will in practice be close to the two-step (or equivalently FE) estimates (as illustrated in Section 8).

Second, the second-step regression makes clear that estimation of the $\gamma$ parameters associated with country-level predictors is based on only $C$ observations, because estimation uses either the coefficients on country-level indicator variables or country means (the dependent variable in (3)). No matter how many individual-level observations $\left(N_{c}\right)$ underlie the calculation of these means, we are effectively using only $C$ observations at the country level (Donald and Lang 2007; Wooldridge 2010, chapter 20).

The small number of countries has several implications. First, the country-level parameters, $\gamma$, are estimated much less precisely than would be suggested by OLS estimation of (1) using all individual-level observations. Ignoring the group-level error results in standard errors that are too small (Moulton 1986).

Second, even if cluster-robust standard errors are used, the assumption that $u_{c}$ is normally distributed is crucial for hypothesis testing because we cannot rely on large sample sizes to provide an asymptotically normal distribution of the parameter estimates. If $u_{c}$ is not normally distributed, tests of statistical significance will not in general be accurate.

small C) . Given the large $N_{c}$, small $C$ structure of most cross-country survey data, OLS (relying on a large $N_{c}$ approximation) appears preferable to GLS (relying on large $C$ approximation). 
Furthermore, even if $u_{c}$ is normal, hypothesis tests and confidence intervals should be based on the $t$ distribution and not the standard normal $(z)$ distribution. ${ }^{7}$ For small $C$, the $t$ critical values are considerably larger than the corresponding $z$ values, implying that standard $z$ tests will find statistically significant results too often. Similar issues arise in the RE approach, as we discuss below.

Third, a small $C$ places a practical limit on the number of variables that can be included in $\mathbf{Z}$. With only a small number of countries, it is impossible to disentangle institutional effects in detail. Even calculating the variance of the country effects is problematic when the number of countries is small. Thus formal statistical inference is difficult. Nonetheless one can always compare the country effects $\hat{v}_{c}$ derived from the first step of estimation using less formal descriptive methods such as exploratory data analysis including graphs. See Bowers and Drake (2005) and the empirical illustration in Section 8 for examples.

The bottom line is that, even with a simple specification of country effects, we need to exercise considerable caution about country-level estimates and hence differences across countries. The two-step approach indicates that the parameters on individual-level predictors $(\boldsymbol{\beta})$ and their standard errors can be estimated reliably. But the regression parameters on country-level predictors $(\gamma)$ and the variance of the country-specific effect $\left(\sigma_{u}{ }^{2}\right)$ are likely to be estimated imprecisely, and so too will their standard errors unless a specific adjustment is made (such as that implicit in the second-step regression). Hypothesis test of the countrylevel parameters is also reliant on the assumption that country effects are normally distributed, which is questionable.

\section{What if the model is complicated further? Country-specific intercepts and slopes}

If there are problems with estimation and inference for a basic model, one would expect problems also to arise if the model specification is made more complicated. We show that this is the case when the basic model specification shown in equation (1) is extended to the more plausible case in which the effects of individual-level predictors differ across countries,

\footnotetext{
${ }^{7}$ As noted above, if the second step is estimated by OLS, standard software will produce $t$-statistics that are correctly referred to the $t$-distribution with $C-k-1$ degrees of freedom (where $k$ is the number of country-level variables).
} 
i.e. there is country-specific variation in the $\beta$. This specification can also be accommodated within the two-step approach. The revised model specification is

$$
y_{i c}=\boldsymbol{X}_{i c} \boldsymbol{\beta}_{c}+\boldsymbol{Z}_{c} \boldsymbol{\gamma}+u_{c}+\varepsilon_{i c}, \text { with } i=1, \ldots, N_{c} ; c=1, \ldots, C .
$$

Observe that $\boldsymbol{\beta}_{c}$ now has a $c$ subscript.

As in the earlier discussion of the interpretation of country-specific random component $u_{c}$, we can either conceptualise the parameters $\boldsymbol{\beta}_{c}$ as being unique and 'nontransferable' (and so fixed), or as being a random draw from a population of possible effects. For the purposes of explaining the two-step method, we assume that both $u_{c}$ and $\boldsymbol{\beta}_{c}$ are random and are uncorrelated with $\boldsymbol{X}_{i c}$ and $\boldsymbol{Z}_{c}$. (We deal with possible dependence of $\boldsymbol{\beta}_{c}$ on $\boldsymbol{Z}_{c}$ below.) Typically $\boldsymbol{\beta}_{c}$ contains multiple scalar parameters $\beta_{j c}$, where $j$ indexes the variables in $\boldsymbol{X}_{i c}$.

The first step of estimation now consists of a separate OLS regression for each country:

$$
y_{i c}=v_{c}+\boldsymbol{X}_{i c} \boldsymbol{\beta}_{c}+\varepsilon_{i c}, \quad i=1, \ldots, N_{c} .
$$

where the regression intercept $v_{c}$ combines both observed and unobserved country characteristics $\left(v_{c}=Z_{c} \gamma+u_{c}\right)$. A second-step OLS regression then yields estimates of $\boldsymbol{\gamma}$ :

$$
\hat{v}_{c}=\alpha+Z_{c} \gamma+n_{c}, c=1, \ldots, C .
$$

where $\hat{v}_{c}$ are the intercept estimates from the first-stage separate country regression. As with the common-slope model, the effects of country-level characteristics are estimated from only $C$ observations, so their standard errors will typically be relatively large and inference has to rely on the assumption of country effects $u_{c}$ being normally distributed.

By contrast, the $\boldsymbol{\beta}_{c}$ estimates from step 1 are based on a large number $\left(N_{c}\right)$ of observations, so we can expect them to be precise, with the correct standard errors and distributed normally. We can easily test for differences across countries in the effects of individual characteristics (e.g. the impact of couples' relative income on the division of housework). Researchers often want to go further and to investigate whether these impacts vary according to country-level factors (e.g. does the impact of relative income on housework depend on the level of gender empowerment in a country?). We can express the dependence of $\boldsymbol{\beta}_{c}$ on $\boldsymbol{Z}_{c}$ as a set of equations, one for each element of $\boldsymbol{\beta}_{c}$ :

$$
\beta_{j c}=\beta_{j 0}+\mathbf{Z}_{c} \boldsymbol{\delta}+v_{j c}
$$

where $\beta_{j c}$ is the $j$ th element of $\boldsymbol{\beta}_{c}, \beta_{j 0}$ is a constant and $v_{j c}$ is the random component of the parameter. This type of formulation is common in the multilevel literature (DiPrete and 
Forristal 1994), and is equivalent to adding interactions between $\boldsymbol{X}_{i c}$ and $\boldsymbol{Z}_{c}$ to the individuallevel equation, as is seen by substituting equation (7) into (5). Using the parameter estimates from the separate country equations in the first step, we can estimate a set of second-step set of OLS regressions based on (7):

$$
\hat{\beta}_{1 c}=\beta_{10}+Z_{c} \boldsymbol{\delta}+v_{1 c}, C=1, \ldots, C .
$$

The two-step set up is again instructive in making explicit the sources of variation in the data that underlie the estimates. Since estimates of $\delta$ are based on only $C$ observations, the same issues which arose in estimating $\boldsymbol{\gamma}$ are also relevant here. Therefore, while we can reliably compare the size of the impacts of individual-level characteristics across countries (because $\boldsymbol{\beta}_{c}$ estimates are based on $N_{c}$ observations), we cannot accurately quantify how these impacts vary with country characteristics (since comparisons are based on $C$ observations). ${ }^{8}$

Models with group-specific intercepts and slopes are usually estimated by REML or FML using multilevel modelling software. As argued earlier, these estimators may have serious limitations when the number of groups is small. Alternative estimation methods such as OLS applied to equation (4) with the predictors supplemented by interactions between $\boldsymbol{X}_{i c}$ and $\mathbf{Z}_{c}$ plus correction of the standard errors, are less applicable to the country-varying slopes case, because the error term now contains a heteroscedastic component in addition to the country-specific effect. The Moulton correction is also inappropriate, although Cameron, Gelbach, and Miller (2008) report that the wild cluster bootstrap-t still performs well when there is heteroscedasticity.

Thus, once again, the two-step method may be a safe and practical alternative. That is, researchers interested in country effects can (i) estimate separate equations for each country, and then (ii) analyse the country-specific components $\hat{v}_{c}$ and $\hat{\boldsymbol{\beta}}_{c}$ in second step regressions. If one is not confident in the suitability of assuming normality at the second stage for inference, one can summarise these differences using less formal and non-inferential descriptive methods.

\footnotetext{
${ }^{8}$ The issue stems from the presence of a country-level random effect. If there were no country-level random variation (no $v_{c}$ in (7) and no $u_{c}$ in (4)), a model described by equation (4) supplemented with interactions of $\boldsymbol{X}$ and $\boldsymbol{Z}$ could be estimated by OLS.
} 


\section{How many countries are required for reliable estimates of country effects?}

In general the statistical properties of standard multilevel estimators are well-defined only when both the number and size of the groups are large. Then, as noted in Section 2, the parameter estimates are consistent and asymptotically normally distributed. If the number of groups is small then, even if the group sizes are large, estimates of the random parameter variances will be imprecise (mirroring what was seen in the two-step approach) and likely to be biased downwards (Hox 2010: 233, Raudenbush and Bryk 2002: 283). The estimates of the fixed parameters will also be affected by the uncertainty in the variance estimates, such that their standard errors are biased downwards and the distribution of test statistics is unknown (Raudenbush and Bryk 2002: 282). ${ }^{9}$

Concrete guidance about the number of groups required to avoid these problems is difficult to find. Most multilevel modelling textbooks mention the issue and sometimes cite rules of thumb (recommending anywhere between 10 and 50 groups as a minimum). However they stress that the minimum number depends on application-specific factors like the number of group-level predictors (Raudenbush and Bryk 2002: 267) and whether interest is focussed on the coefficients on the fixed regression predictors or the parameters describing the distribution of the random effects (Hox 2010: 235). Moreover, advice about sample size is often bound up with considerations of the cost of primary data collection: see Snijders and Bosker (1999: chapter 10). However, these cost issues are not relevant for secondary analysis of the many multilevel country datasets already in existence.

Most analysis of the small group size issue is based on Monte Carlo analysis of simulated data because theoretical analysis cannot provide specific guidance. See for instance the review by Hox (2010: chapter 12). One caveat regarding the Monte Carlo studies is that conclusions are potentially sensitive to model specification, including parameter values and numbers and types of predictors. Previous studies have typically been based on a relatively simple, and mostly linear, models. For example Maas and Hox (2004) specify a linear model for a continuous outcome with a random intercept, a single individual-level regressor (with random slope), a single group-level regressor, and an interaction of the two (both regressors

\footnotetext{
${ }^{9}$ In the special case of balanced data - meaning in the context of equation (1) that $N_{c}$ is the same for all countries and the values of $\boldsymbol{X}_{i c}$ are the same in each country - the fixed parameter estimates are unbiased and standard inference methods, based on the $t$ distribution, can be used even with small samples. However, consistency and inference for the random effects variances still requires large samples. Moreover, the balance conditions (in particular identical values of $\boldsymbol{X}_{i c}$ across countries) are highly unlikely to be met in typical crosscountry applications.
} 
are normally distributed). Austin (2010) specifies a non-linear (logit) model, but with an even simpler specification, consisting of a random intercept and two (joint normally distributed) individual-level regressors. In contrast the Monte-Carlo simulations presented in Section 7 are based on more realistic models including multiple continuous and dichotomous variables constructed to reflect empirical distributions (observed in EU-SILC data). Furthermore, few studies investigate estimator performance with fewer than 30 groups and they typically focus on data with only moderate groups sizes (typically a maximum of 50).

The evidence to date for linear models indicates that OLS, GLS and FML estimates of the parameters associated with fixed predictors $(\boldsymbol{\beta}$ and $\boldsymbol{\gamma})$ are unbiased even if the number of groups is as small as 10 (Hox 2010; Maas and Hox 2004). However, estimates of group-level variances increasingly under-estimate their true values as the number of groups declines.

Recommendations regarding the minimum acceptable number of groups range from about 10 to 100, depending on the estimator and software used (Hox 2010: 234), with REML preferred to FML (or GLS). The standard errors of both the coefficients on fixed predictors and especially the variance parameters are biased downwards when the number of groups is small. Based on their simulation evidence, Maas and Hox's (2004) rules of thumb are: 10 groups are sufficient for unbiased estimates of the $\beta$ and $\boldsymbol{\gamma}$, at least 30 groups are needed for good variance estimates; and at least 50 groups are required for accurate standard error estimates especially for those associated with the random component (co)variance parameters.

There is little evidence for non-linear multilevel models, but the few existing studies suggest similar considerations as for linear models: with a small number of groups, estimates of the fixed parameters remain unbiased but estimates of the random component variances are biased downwards, and the standard errors associated with both fixed and variance parameters are too small. Stegmueller (2013) urges caution in using classical maximum likelihood methods with fewer than 10 or 15 groups, especially when the model includes cross-level interactions and random coefficients, while Moineddin et al. (2007) recommend using at least 50 groups. ${ }^{10}$

\footnotetext{
${ }^{10}$ Moineddin et al. (2007) consider only moderate group sizes (5, 30, 50), so their findings may not be fully applicable to cross-country survey data. For the binary logit model with 30 groups they find little bias of the fixed parameter estimates, except that the estimate of the cross-level interaction parameter is biased upwards (by $5 \%$ ). The variance estimates of the random intercept and random coefficient are biased downwards (by up to 8\%) and non-coverage rates for all parameter estimates, and especially the random components, are too high. Austin (2010), also using logit models with relatively small groups, $N_{C}=5(5) 50$, finds that the fixed parameter estimates are unbiased with as few as 5 groups, but that estimates of the random intercept variance are substantially biased with fewer than 10-15 groups (depending on the estimation method used). Non-coverage
} 
Recent econometrics literature has examined how well corrections to OLS standard errors perform when the number of groups is small. Both cluster-robust and block-bootstrap standard error estimates are valid only asymptotically and tend to be too small when there are few groups (Cameron, Gelbach, and Miller 2008). The Moulton (1986) correction may not work well either, since the within-group correlation tends to be underestimated when there are few groups (Angrist and Pischke 2009). Cameron, Gelbach, and Miller (2008) report Monte Carlo simulations showing that all three types of standard error estimate are much too small when the number of groups fall below 25. They also show that for cluster-robust and Moulton-corrected standard errors, the bias is worse for group-level parameters than those associated with individual-level variables (specifically, the bias is larger for variables with larger intra-group correlations).

It is clear that the number of countries in the multilevel country datasets typically available falls within the risky range identified by these Monte Carlo studies. Can anything be done to increase the reliability of the estimates?

There does not appear to be an easy solution using the multilevel model estimation commands in standard software. Most software, including Stata (personal communication from R. Gutierrez, StataCorp, 17 December 2009), does not routinely make small-sample adjustments to estimates of confidence intervals or test statistics. An exception is HLM (Raudenbush et al. 2004, cited in Hox 2010), which uses the $t$ distribution with degrees of freedom based on the number of groups (similar to the second-step estimation outlined above) and should give better inference for the fixed parameters. More specialist corrections (for linear models only) have also been developed but only implemented in a few software packages. A small-sample correction to the REML estimator is available to improve the inference for the fixed parameters (Kenward and Roger 1997, 2009), and has been implemented in SAS. Bootstrapping methods may reduce bias and improve inference for the random effect variances as well when there are few groups or the random effects are not normally distributed. ${ }^{11}$

rates for the parameters on individual-level regressors are within expected bounds even with 5 groups. Austin does not report non-coverage rates for the random intercept variance and there is no group-level regressor. Stegmueller (2013) explicitly considers a cross-country data structure but focuses on the fixed parameters. Using a probit model, he finds that the estimates are subject to little bias even with only 5 groups, except (as in Moineddin et al. 2007) for a model including a cross-level interaction with a random coefficient: in this case the estimate of the fixed cross-level interaction parameter is biased upwards by $15 \%$ with 5 or 10 groups. However, the non-coverage rates are too large (by at least 5 percentage points) for most fixed parameter estimates with 10 groups or less.

${ }^{11}$ For example, see the option in MLwiN based on Carpenter et al. (2003), with SAS macros provided by Wang et al. (2006). However, the method may yield coverage rates that are far from satisfactory. E.g. there are 
If pooled OLS is the estimation method, with a focus on estimating the fixed parameters, there are potential improvements but at the cost of complexity. For example, cluster-robust standard errors can be further corrected using so-called bias-reduced linearization (Bell and McCaffrey 2002; Angrist and Pischke 2009: 320). An alternative bootstrap technique, the 'wild cluster bootstrap-t', also seems to perform well in small samples (Cameron, Gelbach, and Millar 2008), although it produces only t-statistics and not standard errors. Recent studies have indicated that a simple rescaling of the cluster-robust standard errors, and the use of critical values from the $t$-distribution, may deliver reliable test results (Bester et al 2011, Brewer et al 2013). Finally, the two-step approach we have presented is also a viable estimation method that will offer improved inference at the country level (Donald and Lang, 2007).

For most of these methods, the fact remains that in the small- $C$ case, one has to assume that country-level effects $\left(u_{c}\right)$ are normally distributed in order to derive good estimates of the standard errors of country-level regression parameters $(\gamma)$ and the variance parameters $\left(\sigma_{u}^{2}\right)$ and hence to do statistical inference. If the normality assumption cannot be justified, bootstrapping methods may provide acceptable inference. Alternatively, and especially if the country effects are considered to be fixed rather than random, then, as we discuss below, the option that remains is to use less formal descriptive methods to describe step-1 estimates of cross-country differences (Bowers and Drake, 2005).

\section{Further complications: non-linear models}

In many applications the outcome variable is binary rather than metric. To allow for this we reinterpret the outcome variable in equation (4) as a latent index, $y_{i c}{ }^{*}$, and the observed outcome $y_{i c}$ is a binary variable equal to one if the index is non-negative, and equal to zero if negative:

$$
\begin{aligned}
& y_{i c}{ }^{*}=\boldsymbol{X}_{i c} \boldsymbol{\beta}_{c}+\boldsymbol{Z}_{c} \boldsymbol{\gamma}+u_{c}+\varepsilon_{i c} \text {, with } i=1, \ldots, N_{c} ; c=1, \ldots, C \text {. } \\
& y_{i c}=1 \text { if } y_{i c} *>0 \\
& =0 \text { if } y_{i c} * \leq 0
\end{aligned}
$$


As with any random effects binary dependent variable model, parameters are identified up to a scale factor only and, for identification, it is conventional to normalise $\sigma_{\varepsilon}^{2}$ to equal $\pi^{2} / 3$ in a logit model and one in a probit model. The choice between the two models is not usually important and to some extent depends on disciplinary traditions (the probit model is common in economics while researchers in other disciplines, especially sociology, tend to prefer the logit). Our examples focus on the logit model.

All four methods presented in Section 2 are available for non-linear models. Thus we could estimate a pooled logit (with clustered standard errors), separate logits for each country, a FE logit (including indicator variables for the country intercepts), or a RE logit. Unlike linear models, estimation typically involve types of maximum likelihood techniques (relying on large sample sizes for desirable properties of estimators), and so it is possible that non-linear models are more sensitive to small sample sizes.

The two-step approach can also be applied to binary response models as long as the number of individual units per country is large: see Borjas and Sueyoshi (1994) and the application of Huber et al (2005). See also Wooldridge (2010: chapter 20) who argues that the approach is applicable to any nonlinear model with a linear index structure. The first step consists of logit (or probit) regression using separate logit regressions to the data for each country. (Alternatively, one could pool the data from all the countries and fit a model with country-specific intercepts.) Because there are many observations per country, the estimated parameters on the individual-level predictors, $\boldsymbol{\beta}_{c}$, are consistent. The linear index structure implies that the country-level intercepts and coefficients can be expressed as a linear function of the country variables $\boldsymbol{Z}_{c}$ exactly as in a fully linear model (equation (7) for example). The second stage estimation is therefore identical to the linear model: the estimated country intercepts and coefficients are regressed on country-level variables using OLS.

As with the linear model, the two-step approach underlines the importance of the number of countries for reliable estimates of the parameters describing country effects, $\gamma$, and their standard errors. Using Monte Carlo analysis, Borjas and Sueyoshi (1994) explore the consequences of different values of $C$ and $N_{c}$. In so far as one can generalise from the particular specification used in their analysis, it appears that having only 10 groups is definitely problematic for estimation and inference but, as long as $C$ is 25 or more (and $N_{c}$ is large), country effect estimates are less problematic (they have reduced bias and better coverage probabilities): see Borjas and Sueyoshi (1994: Table 4). 


\section{How many countries are needed for good estimates? Monte-Carlo simulation results}

We use Monte-Carlo simulations to assess how large the number of countries needs to be in order to derive accurate estimates of model parameters and their standard errors from the standard multilevel model estimators. Simulation methods have also been used by other authors to assess multilevel model estimates, but for several reasons their results do not necessarily translate to typical cross-country applications. ${ }^{12}$ First, these previous studies have mainly been concerned with applications to education and health research that involve moderate numbers (a few tens) both of groups and numbers of observations within groups. Thus they do not usually consider the sample sizes of most relevance to cross-country researchers, i.e. a number of groups below about 30 and group sizes in the hundreds (at least). Second, there has tended to be a focus on linear models, while many socio-economic outcomes of interest call for non-linear (e.g. logit) methods. Third, to our knowledge, all previous studies use very simple, rather unrealistic, model specifications, typically including only two or three 'well-behaved' (normally distributed) regressors. We include binary, categorical, and continuous variables, and do not impose normality.

Our work addresses these issues to provide a more comprehensive treatment of the performance of multilevel methods using cross-country survey data. We consider both linear and non-linear models using data structures that are similar to those found in multi-country data sets, we employ a greater range in the number of countries, and we also give greater attention to simulation variability than previous research - this turns out to be relevant when assessing the properties of estimates of some individual-level and country-level effects (see below).We conclude that with 10 or fewer countries, researchers are likely to under-estimate the sizes of the country random effect variances to an unacceptable degree. Estimates of the fixed parameters are generally unbiased but they may be imprecise, particularly if associated with country-level factors. Moreover, researchers are likely to find significant results too

\footnotetext{
${ }^{12}$ Maas and Hox (2005) consider a linear model with a random intercept, a country-level regressor with random slope, and a cross-level interaction term. They consider designs with combinations of $C=30,50,100 ; N_{C}=5$, 30,50 ; ICC $=0.1,0.2,0.3$. Moineddin et al. (2007) consider a very similar design and regressors, but for a binary logit model. Austin (2010) considered a mulitlevel logit model with random intercept, and designs with combinations of $C=5(1) 20 ; N_{C}=5(5) 50$. Simulations by Browne and Draper (2000) and Pinheiro and Chao (2006) re-used the three-level data structure employed by Rodriguez and Goldman (1995) with relatively small $N_{C}$. The simulation design of Stegmueller (2013) is the closest to ours in that he uses combinations with $C=$ 5(5)30; $N_{C}=500$; ICC $=0.05,0.10,0.15$, and he considers multilevel linear and non-linear binary models (but probit rather than logit ones). Unlike us, Stegmuller highlights the contrast between Bayesian and frequentist methods and his data generating process is less like those found in typical multi-country country data sets.
} 
often when conducting hypothesis tests of either the random effect variances or the fixed parameters (especially those associated with country-level factors). We conclude that to have full confidence in the results, researchers will probably want to use at least 25 countries for linear models and 30 countries for non-linear models.

Our simulation results are based on linear and non-linear two-level models, with two versions of each: a basic specification with random intercepts (Basic) and an extended specification with random intercepts and slopes (Extended). The model specifications are chosen to represent those that analysts have fitted to multi-country data, and are inspired by the EU-SILC data used in our numerical illustration in Section 8. Given this link, we refer to the outcome variables for the linear and non-linear models as 'hours' (of work) and 'participation', respectively. For each of the four models, our simulations hold the number of individuals per country, $N_{C}$, fixed at 1000 , and vary the number of countries, $C$, from 5 to 50 in intervals of 5 , and also consider $C=100$ in order to have a reference point for a case in which researchers would agree that $C$ is large.

In the Basic Model, the regressors include a constant (intercept), individual-level predictors with fixed slopes, a country-level predictor, and a random country intercept. (The model also includes an individual-specific error term.) To maintain the link with our EUSILC application, we refer to the individual-level predictors as age (continuous), agesquared, cohab (whether married or cohabiting; binary), nownch (number of own children; integer), isced (educational level; four categories with the lowest excluded from the regressions). The country-level fixed is chexp (country spending on childcare and preprimary spending as a \% of GDP, continuous). The Extended Model includes the same regressors but adds two cross-level interactions (between chexp and cohab, and chexp and nownch), and two random slopes (on cohab and nownch). In common with most social science applications, we assume that the random effects are uncorrelated with each other. The models are summarized in Table 2.

Compared to previous Monte-Carlo simulations of multilevel models, our specifications include a greater number of regressors and different types of variables. For example, the model used in the oft-cited Maas and Hox (2005) study included only one individual-level regressor and one country-level regressor (both of which were continuous, normally distributed, variables). By including a more realistic set of regressors, we can be more confident that the performance of the estimators will hold up in practical applications and does not depend on the simplicity of the experimental specification. Furthermore we chose the parameters to correspond with parameters estimated by fitting the Basic and 
Extended models for hours and participation probabilities to EU-SILC data for 2007 on women aged 18-64 years from 26 countries: see Table 2 for the values used. The value of the intra-class correlation (ICC) is relatively small in each of the four cases, which is common finding in the multi-country data context. ${ }^{13}$ We specified the joint distribution of the regressors by exploiting the fact that each combination of regressor values defines a cell with an associated probability of occurrence. We derived the cell probabilities from the empirical frequency distributions in the 2007 EU-SILC estimation samples cited earlier (separately for the hours and participation models), and then generated data sets reflecting these distributions for each value of $C$ (and for each model) using a random number generator. ${ }^{14}$ In common with other simulation studies of multilevel models, the joint distribution of the regressors is the same across replications.

All estimation and simulation was undertaken using Stata (StataCorp 2011). ${ }^{15}$ The models for hours were estimated by maximum likelihood using the xtmixed command's REML estimator. The models for participation were estimated by maximum likelihood using the xtmelogit command's adaptive Gaussian quadrature procedure (with seven integration points). The number of replications for each model, $R$, was chosen to be as large as possible in order to reduce simulation variability while also taking into account estimation time which is longer for non-linear models than linear models, and the more complex the model that is estimated. Our choices for $R$ were 10,000 for the Basic hours model, 5,000 for both the Extended hours model and the Basic participation model, and 1,000 for the Extended participation. A very small number of replicate estimations did not converge within the maximum of 250 iterations that we specified (at most approximately $0.02 \%$ per model) and, as is usually done, we exclude these estimates from our simulation summaries.

The simulations were designed to examine the accuracy of the estimates of model parameters (fixed effect coefficients and random effect variances), and also of their standard errors and hence inference regarding the statistical significance of the various effects. We report three summary measures:

\footnotetext{
${ }^{13}$ We did not vary the values of the ICC across simulations as previous research suggests that this has little effect on results (see e.g. Maas and Hox 2005).

${ }^{14}$ To construct the cells, age was grouped into five categories derived as follows. In EU-SILC data, we first fitted either a Singh-Maddala distribution (hours models) or a uniform distribution (participation models). The fitted parameters were used to generate values of age between 18 and 64 in the simulated data (values used in the regressions). They were grouped into five categories in order to incorporate age into the cell-based approach. ${ }^{15}$ Stata do files are available from the authors on request. We used Stata version 11 (on a desktop PC and a network server running Windows) for most of the simulations; version 12 was used for the simulation summaries.
} 
Relative parameter bias: defined as the percentage difference between estimated parameter and the true parameter at each replication, averaged over $R$ replications. Ideally, relative bias equals $0 \%$ for each parameter.

Relative standard error bias: we compare the standard error reported by the software to the standard error that we calculate from the variation observed in the parameter point estimate during the simulation. More formally, the 'analytical' standard error is the reported standard error averaged over $R$ replications, and the 'empirical' standard error is the standard deviation of the estimated parameter that we calculate based on the same $R$ replications (Greene 2004). We define the relative standard error bias as the percentage difference between the analytical and empirical standard errors, assuming the empirical standard error is an accurate estimate of the true standard error. ${ }^{16}$ Ideally, the relative bias equals $0 \%$ for each standard error. Non-coverage rate: to assess overall inference, we calculate a 95\% confidence interval (CI) for each estimated parameter, assuming normality (Maas and Hox 2005: 89). A non-coverage indicator variable was set equal to zero if this CI included the true parameter and one if it did not. The average over $R$ replications of this variable is the non-coverage rate. Ideally, the non-coverage rate for a $95 \% \mathrm{CI}$ is 0.05 . Rates larger than 0.05 indicate that the estimated CI is too narrow.

Most simulation studies of multilevel models report parameter bias and non-coverage rates only, and often interpret non-coverage rates as indicating the accuracy of the standard errors. However, non-coverage depends on a combination of parameter bias, the distribution of the parameter estimates (usually assumed normal) and the accuracy of the SEs. For example, even with accurate SEs, non-coverage will tend to exceed 0.05 if the parameter estimate is biased. To give a fuller picture of the potential sources of unreliability, we report estimates of SE bias in addition to non-coverage rates.

Since the relative bias measures and the non-coverage rates are themselves estimates (they are both means over replications), they are subject to simulation variability - as

\footnotetext{
${ }^{16}$ For parameter $\theta$, the empirical SE is $s_{e}(\hat{\theta})=\sqrt{1 /(R-1) \sum_{j=1}^{R}\left(\hat{\theta}_{j}-\overline{\hat{\theta}}\right)^{2}}$ and the analytical SE is $s_{a}(\hat{\theta})=$ $1 / R \sum_{j=1}^{R} \operatorname{se}\left(\hat{\theta}_{j}\right)$, where $j$ indexes replications and $s e\left(\hat{\theta}_{j}\right)$ is the reported standard error for parameter estimate $\hat{\theta}_{j}$. A caveat is that if the square of the empirical SE, $s_{e}^{2}(\hat{\theta})$, is an unbiased estimate of the true variance of the parameter estimate, $\sigma^{2}(\hat{\theta})$, it does not follow that, after taking square roots, $s_{e}(\hat{\theta})$ is also an unbiased estimate of the true standard error $\sigma(\hat{\theta}): s_{e}(\hat{\theta})$ will tend to underestimate $\sigma(\hat{\theta})$ (by Jensen's inequality). Since we find that the $s_{a}(\hat{\theta})$ tends to be smaller than $s_{e}(\hat{\theta})$ (for small numbers of countries), our estimates of the (negative) relative standard error bias may be understated.
} 
emphasized by Cameron and Trivedi (2010: section 4.6). ${ }^{17}$ We summarize this variability by presenting the $95 \% \mathrm{CI}$ for estimated relative parameter bias and non-coverage rates. ${ }^{18}$ Although this is not commonly done, it highlights some interesting features of estimates, especially of country effects: see below.

The simulation results are summarised in Figures 1-10. For the Basic models, we present the relative bias of the parameter estimates and of the standard errors, as well as the non coverage rate, in Figures 1-3 (hours) and Figures 6-8 (participation). For the Extended models, we present the relative parameter bias and non coverage in Figures 4 and 5 (hours), and Figures 9 and 10 (participation). All results are provided in tabular form, with additional details, in the Appendix. For brevity, the results for some of the individual-level fixed parameters are excluded.

\section{Simulation results: linear model}

For the linear model with a random intercept and a country-level regressor (Basic model for hours), we find that the individual-level variance component and almost all the fixed parameters are unbiased regardless of $C$. In Figure 1, relative bias for sig_e, cohab, nownch, and age, is close to zero, with little simulation variability. The results for country-level regressor (chexp) stand out, however, as there is substantial simulation variability in relative bias even for large values of $C$. To be sure, the $95 \%$ CI for relative bias includes zero for all values of $C$ (except $C=20$ ) but, even for $C=50$, the $C$ I ranges from $-15 \%$ to $+14 \%$. The implication is that, although the country-level coefficient is unbiased in expectation, there is substantial uncertainty associated with the estimate of relative bias. This stems from the relatively small number of countries underlying the estimates. Relative bias for the countrylevel coefficient is greater than reported by Stegmueller (2013: Figure 2) for most values of $C$. We presume that the differences arise because we use a more complicated (and more realistic) data generating process than he uses. The country-level variance (sig_u) is underestimated but the bias falls rapidly with the number of countries, from $8 \%$ for $C=5$ to around

\footnotetext{
${ }^{17}$ The CIs are closely related to the empirical standard error, $s_{e}(\hat{\theta})$, e.g. the standard error of the relative parameter bias is $(100 / \theta) s_{e}(\hat{\theta})$. Another measure of estimator inaccuracy is the mean squared error (MSE), defined as $E\left[(\hat{\theta}-\theta)^{2}\right]$. It can be shown that MSE $=\sigma^{2}(\hat{\theta})+[\operatorname{bias}(\hat{\theta})]^{2}$, thus it reflects inaccuracy stemming from both imprecision and bias. We do not report MSE because in our simulations the variance component dominates the (squared) bias, and so parameter inaccuracy, as would be measured by MSE, is almost fully captured in our CIs.

${ }^{18}$ For clarity in Figures 2 and 7, we do not present the CIs around the estimates of relative standard error bias.
} 
$1 \%$ or less for $C \geq 20$. This is consistent with Maas and Hox (2004: 135) who report a bias of $25 \%$ with 10 groups but negligible bias for 30 or more groups.

The relative bias of the standard errors for the Basic linear model for hours is shown in Figure 2. For chexp, the standard error is underestimated by $8 \%$ for $C=5$ but the bias declines to under $2 \%$ for $C \geq 15$. For the country-level variance, there appears to be negligible bias in the standard errors for almost all values of $C$. Even for $C=5$, the standard errors are downward biased by only 3\%. The corresponding non-coverage rates are shown in Figure 3. Rates are estimated to be close to the nominal rate of 0.05 at all values of $C$, for the individual-level variance and for all the fixed parameters except chexp. For chexp, as expected from the under-estimated standard errors, non-coverage rates are markedly greater than 0.05 when $C$ is very small, but they reach around 0.06 for $C \geq 20$. Rates diverge to a greater extent for the country-level variance. It is only for $C>35$ that the non-coverage rate is within one percentage point of 0.05. Since the standard errors are unbiased for sig_u, the high non-coverage rates at small $C$ stem from parameter bias (Figure 1) or from a non-normal distribution of parameter estimates.

Figures 4 and 5 summarize the results for the Extended model for hours, now including a cross-level interaction and two random slopes. Compared to the results about bias for the simpler model, the main change compared to Figure 3 is the greater prevalence of simulation variability in estimates of bias for the fixed parameters with the exception of that for age. (Having a relatively small number of countries now has implications for estimates of cross-level interaction effects, as well as for the country-level effect itself; it is not simply that the number of replications is smaller.) Nonetheless, relative bias is less than $2 \%$ for values of $C>10$, and the $95 \%$ CI is $-2 \%$ to $+2 \%$ for all but one of the cross-level interaction effects (chexpXnownch) for $\mathrm{C}>30 .{ }^{19}$ The random slope and country-level variances are all under-estimated, but the downward bias is less than $2 \%$ as long as $C \geq 25$.

Figure 5 shows that non-coverage rates are generally too large for all parameters except the age effect. Compared to the simpler linear model, this is apparent for more of the fixed parameters. As before, the explanation is that having a relatively small number of countries has implications for the standard error estimates of effects in addition to those for

\footnotetext{
${ }^{19}$ This bias is greater than reported by Maas and Hox (2005: 89) who cite a maximum bias in effect coefficients of less than $0.05 \%$ for $C \geq 30$ in a model with country- and cross-level interaction effects. In contrast the relative bias at small $C$ is less than reported by Stegmueller (2013: Figure 5): e.g. about $-10 \%$ for $C=10$.
} 
the country-level intercept, transmitted via the cross-level interactions or random slopes. ${ }^{20}$ Non-coverage rates generally decrease as the number of countries increases, dropping sharply between $C=5$ and $C=20$, for both fixed parameters and random effect variances. What counts as the appropriate number of countries depends on how accurate one wishes one's standard errors to be. Insisting on a non-coverage rate within one percentage point of 0.05 would imply having 35 or more countries. With $C=20$, the non-coverage rate is around 0.07 to 0.08 depending on parameter (with some variability around those values).

\section{Simulation results: non-linear (binary logit) model}

In Figures 6-8, we summarise results for the Basic logit model for participation. The smallsample properties of this model are less well-known than for the linear model, and so the simulations are of particular relevance. As it happens, there are some similarities with the results for the corresponding linear model. Figure 6 shows that the relative bias in the fixed parameters is near zero for almost all values of $C$. The main difference from Figure 1 is that there is now relatively little simulation variability in the country-level effect; instead there is now relatively substantial variability in the estimate of bias in the effect of cohab. For this particular effect, there is marked downward bias in the estimated effect at values of $C<20$, though also observe that the CIs for relative bias include zero at all $C$ values. The country variance (sig_u) is downwardly-biased, also as before, but now to a greater extent than in the Basic linear model. It is only for $C \geq 30$ that the bias is less than $5 \%$.

The estimated bias of the standard errors is summarized in Figure 7. There is little standard error bias for the fixed parameters associated with individual-level predictors. However the standard errors of the fixed parameter at country level, chexp, and of the country-level random intercept variance, $s i g \_u$, are substantially under estimated for small values of $C$. These biases exceed those of the linear model (Figure 2). Only for $C \geq 25$ does the bias fall below $5 \%$ for chexp ( $C \geq 20$ for sig_u).

Non-coverage rates for the Basic logit model are shown in Figure 8. As for the Basic linear model (Figure 3) and, mirroring the negligible bias of the standard errors, noncoverage rates are close to 0.05 for the fixed parameters of individual-level predictors. Again,

\footnotetext{
${ }^{20}$ We also simulated a model with cross-level interactions but without random slopes. The non-coverage rates for the fixed effects associated with the cross-level interactions and their corresponding individual-level predictors (chexpXnownch, nownch, chexpXcohab, cohab) were all close to 0.05, suggesting that excessive noncoverage at small $C$ stems from the presence of random components.
} 
the exceptions are the fixed country-level effect and the country-level intercept variance. For chexp, non-coverage rates are higher than in the linear model case. Only for $C=40$ does the non-coverage rate for chexp get to within one percentage point of 0.05. But if one were prepared to tolerate a non-coverage rate of 0.08 , then having $C>20$ would suffice. Similarly, the non-coverage rate for the country-level variance also much too high for most $C$ values and by a greater amount than in the corresponding linear model case (note the vertical axis scale in this case). For $C=30$, the non-coverage rate is around 0.10 , i.e. twice the nominal rate of 0.05 . Even when $C=100$, the non-coverage rate is around 0.07 .

The results for the Extended logit specification also parallel those for the corresponding linear model and, again, the accuracy of corresponding estimates is less, for both parameters and standard errors. The patterns of relative bias shown in Figure 9 are similar to those shown in Figure 4, in the sense that simulation variability is relatively large for all of the estimates of bias in the fixed parameters.$^{21}$ Again, however, virtually every CI for the relative bias estimates includes zero, and for all $C$. And, for all fixed parameters except that for cohab, the relative bias estimate itself is no more than $2 \%$ as long as $C \geq 20$. (By contrast, the estimated relative bias for cohab is around $-7 \%$ when $C=100$.) The random slope and intercept variances are substantially under-estimated when the number of countries is small. For example, the random slope variances are around half the true value for $C=5$, though 'only' $90 \%$ of their true value for $C=20$. Relative bias falls to $-5 \%$ or less only if $C$ is around 40 . For the country variance, this degree of bias is achieved if $C \geq 30$.

The picture for non-coverage rates shown in Figure 10 is also broadly similar to that for the corresponding linear model (Figure 5). Simulation variability is larger (partly reflecting the smaller number of replications), but non-coverage rates are also larger, especially at small values of $C$. Even with $C \geq 35$, the non-coverage rate is greater than 0.06 for several fixed parameters. On the other hand, if one is prepared to tolerate a non-coverage rate up to 0.08 , the simulations suggest that having at least 25 countries would suffice. To generate the same non-coverage rate for the random coefficient variances appears to require around 30 countries or more, whereas for the country variance, more than 35 are required. The results suggest that to lower the rate further would require a very large number of countries,: even when $C=100$, the non-coverage rate is greater than 0.06 , for all three variances.

\footnotetext{
${ }^{21}$ Observe the different vertical axis scales in Figure 9. In part, the greater simulation variability for the logit model also reflects the smaller number of replications: 1,000 rather than 5,000.
} 
Our simulation analysis endeavours to provide practical answers to the question of how large the number of countries needs to be for multilevel model analysis of multi-country data. We have demonstrated that, with 10 or fewer countries, estimates of parameters and their standard errors are inaccurate to what is surely an unacceptable degree, with substantial under-estimates of country random effect variances and excessive non-coverage rates for both fixed and random effects. But how many countries are required? Our own practical ruleof-thumb would be: at least 25 countries for linear models and 30 countries for non-linear models. However, we would also stress that there is no single 'magic' number of countries the number depends on a number of factors. We have highlighted, for instance, that the number consistent with derivation of accurate estimates depends on a researcher's definition of acceptable accuracy. We have used a relative bias of $0 \%$ and a non-coverage rate of 0.05 as reference points, but have shown how fewer countries are sufficient if one is content to be merely fairly 'close' to these ideals.

We have also demonstrated that the appropriate number of countries depends on what model is being estimated and which effects that the researcher is primarily interested in. At one extreme, it is well-known that for a linear model REML produces unbiased estimates of the effects of fixed individual-level covariates and our simulations confirm this. But our simulations have also shown that unbiasedness may coincide with a substantial degree of estimate variability particularly for effects associated with country-level factors (country effects and cross-level interaction effects), reflecting the small number of countries relative to the number of individuals per country. What is true on average across repeated estimation need not be true in a single estimation instance using a particular data set (the situation faced by the practising researcher).

More positively, we have shown that non-coverage rates for fixed parameters in linear models are relatively good, as long as the number of countries is greater than around 25 . With this number of countries, linear model estimates of random effect variances and their standard errors also appear to be accurate to an extent that may satisfy many practising researchers.

Our simulation results for the binary logit mixed models regarding relative bias and non-coverage have many parallels with those for the corresponding linear models. The primary difference between models is that a greater number of countries appears to be necessary to generate the same degree of accuracy in parameter estimates and standard errors, 
other things being equal. In particular for random coefficient variances (if specified) and especially the country-level variance, at least 30 to 35 countries may be required to derive sufficiently accurate estimates - which is more than is usually available (see Table 1).

An additional warning concerning non-linear mixed models in general and the binary logit mixed model in particular is that the estimator used for maximization also matters. We have used adaptive Gaussian quadrature, which has been found to produce more accurate estimates than penalized quasi-likelihood (Rodriguez and Goldman 2001, Pinheiro and Chao 2006, Austin 2010). Other researchers have shown that Bayesian estimation methods using Markov chain Monte-Carlo methods also perform well, especially with a relatively small number of ‘countries’ (Austin 2010; Browne and Draper 2000, 2006; Stegmueller 2013).

\section{Empirical illustration: hours worked and work participation}

Following the Monte Carlo analysis, and to illustrate the practical consequences of using multilevel data with a small number of countries, we present a simple but representative application based on data from a commonly-used dataset, EU-SILC. ${ }^{22}$ Since the Monte Carlo simulations indicated that we could expect substantial problems with around 10 countries, we randomly selected 10 from the available 26 countries. ${ }^{23}$ We estimate models of the form specified in Table 2, that is linear models of working hours and non-linear (binary logit) models of work participation. We focus the discussion here on the Basic specification containing country-specific intercepts but common slopes across countries. (Results for an Extended specification are available from the authors on request.)

\section{Linear model}

The dependent variable in the linear model is the usual number of total weekly hours worked in the main job of person (woman) $i$ in country $c$ and the explanatory variables are: $a g e_{i c}$ and age-squared $_{i c}$; cohab $_{i c}$, a dummy variable indicating whether a woman is married/cohabiting; nownch $_{i c}$, number of own children; three isced ${ }_{i c}$ dummy variables indicating highest

\footnotetext{
${ }^{22}$ We use data from 2007, $4^{\text {th }}$ release, which contains 26 countries: the 27 EU member states excluding Bulgaria, Malta and Romania, plus Iceland and Norway.

${ }^{23}$ The 10 countries are: Denmark, Estonia, Germany, Hungary, Finland, France, Netherlands, Poland, Portugal, United Kingdom.
} 
educational level according to the International Standard Classification of Education $(\text { ISCED })^{24}$; and $c h \exp _{c}$, the total childcare and pre-primary spending as a \% of GDP in country c. The model is estimated using 45,464 observations on working women aged 18-64 from 10 countries.

Table 3 and Figure 11 summarise the estimates of the Basic hours model using the different methods. ${ }^{25}$ The table lists the fixed parameter estimates and standard errors associated with selected variables (cohab, nownch and chexp) as well as (when estimated) the standard deviations of the random country intercept (sig_u), the individual-level error (sig_e) and the intra-class correlation (ICC). Where appropriate we use the two-step estimates as a benchmark for the other multilevel methods. Figures 11 and 12 (discussed below) graph the estimates of the country intercepts from step 1 of the two-step method.

Beginning with the fixed parameters, we see that all the methods indicate that partnered women work about one hour per week less than single women, that having an extra child is associated with about a one hour reduction in work time, and that more childcare spending in a country is associated with more hours of work (although this last effect is only statistically significant in one case). However, as expected from the discussion in Sections 2 and 3, and the Monte Carlo simulations, there are some notable differences across methods.

The first striking difference is that the OLS coefficients (methods 1 and 2) differ substantially from those of the other estimators (3-7) and in particular from the two-step benchmark. For example, being partnered is associated with 1.7 fewer hours of work according to OLS but with 1.2 fewer hours according to the two-step method. Also, a one percentage point difference in childcare spending (as a proportion of GDP) is associated with 1.9 hours more work according to the OLS estimates but only 0.4 hours (and not statistically significant) according to the other methods. The differences may reflect that, unlike the other methods, OLS ignores the unobserved country effect and so gives too much weight to between-country variation and not enough weight to within-country variation.

A second feature of the OLS estimates is that the use of cluster-robust variances (method 2) leads to standard errors which are much larger (by about 5-25 times) than OLS standard errors (method 1 ). We expected the clustered standard errors to be larger, because

\footnotetext{
${ }^{24}$ ISCED level 3 is upper secondary (usually post-compulsory education from 15 or 16 years), level 4 is postsecondary but non-tertiary, and levels 5-6 are tertiary education (first and second stage). The default category combines ISCED levels $0-2$ (the various stages of compulsory education).

${ }^{25}$ We omit separate models for each country because the parameters from such unrestricted models are not readily comparable to models in which the parameters are restricted to be the same (or vary only parametrically) across countries.
} 
they account for within-country correlation across individuals, but we also noted above that cluster-robust methods may not work well with only a few countries. This warning appears to be borne out by a comparison of the clustered standard errors with those from the other methods that take proper account of the multilevel data structure. In particular, the standard errors associated with the two individual-level variables from methods 3-7 are almost the same as the (uncorrected) OLS standard errors. This suggests that clustered standard errors at the individual level may be too large, rather than too small, when number of countries is small. ${ }^{26}$ In contrast, the clustered standard errors at associated with the country-level regressor are much closer to those from the other methods.

Next, we compare the fixed parameters estimated by methods 3-7 which account explicitly for the multilevel structure of the data. We take the two-step estimates (method 7) as a benchmark (noting that step 1, method 7a, is identical to the country FE approach, method 3). The point estimates of the fixed parameters, both for individual- and country-level regressors, are almost identical across the methods and the same as the two-step method, consistent with them being unbiased. The Monte Carlo simulations of the REML estimator indicated that the fixed parameter estimates were unbiased even for small group sizes.

We also see that the three random effects estimators (GLS, REML and FML) yield estimates of the effects of the individual-level predictors which are identical (to three decimal places) to the FE estimates. We expected GLS and FE to be very similar because, given the large number of individual observations and few countries, the GLS estimator uses almost entirely within-country variation. In addition, the ML estimates of the random effects model (methods 5-6) are very close to RE GLS. An implication is that, for estimating the fixed parameters associated with individual-level predictors, it does not matter whether the country intercept is modelled as fixed or random.

The Monte Carlo simulations indicated that there should be little bias in the standard errors of the parameter estimates on the individual-level predictors, but some downward bias in the standard error of the parameter estimate of the country-level predictor (although only about $2 \%$ at $C=10$, Figure 2). Consistent with expectations, the standard errors of the individual-level fixed parameters are identical across methods 3-7. But for the country-level predictor, chexp $_{c}$, the standard error is 5.35 using step 2 of the two-step (method $7 \mathrm{~b}$ ), compared to 4.79 using REML (method 6). This represents a difference of some $11 \%$ (rather

\footnotetext{
${ }^{26}$ A caveat is that cluster-robust standard errors allow for a more general form of within-country correlation than implied by a country intercept common to all individuals.
} 
larger than expected) and would lead to higher likelihood of finding a significant effect of child expenditure using REML rather than the two-step method (although in this example both are insignificant at conventional significance levels). Of the other estimators, FML gives the same standard error for the $\operatorname{chexp}_{c}$ parameter as REML, but GLS gives a slightly larger standard error even than the two-step method.

The final group of estimates are the variances of the random components. The Monte Carlo simulations and earlier research suggest that the estimate of sig_e should be unbiased but that sig_u should be underestimated (perhaps by some 3\%, Figure 1), leading to underestimates of ICC. Methods 3-7 give identical estimates of sig_e. Step 2 of the two-step (method 7b) gives a benchmark estimate of sig_u of 4.86, while the REML and FML estimates are both 4.34 (11\% smaller) and the GLS estimate is 4.87 ( $0.3 \%$ larger). Again the difference between the two-step results and the REML is somewhat larger than was expected from the Monte Carlo experiments. There is a corresponding difference between ICC, estimated as 0.195 by the two-step method (or the almost identical GLS) and as 0.162 by REML and FML. Finally, the Monte Carlos also indicated that the standard error of the sig_u estimate would be too small. Both REML and FML produce the same estimate, but we are unable to compare it with GLS or step 2 of the two-step because Stata does not report estimates of the standard errors of variance components in linear models.

In view of the small number of country-level observations, an alternative to estimating the effects of country-level predictors statistically is to use less formal descriptive or visualisation techniques at the country level (Bowers and Drake 2005). This approach amounts to replacing step 2 of the two-step method with graphs of the estimated country intercepts (from step 1 or country FE) or with a verbal description of the differing country intercepts in terms of national institutions. In Figure 11 we plot the estimated country intercepts against childcare spending (these are the data points used in the step 2 regression). Consistent with the statistically insignificant chexp estimate of Table 3, there does not appear to be a systemic association between childcare spending and the country average level of work hours (adjusted for national differences in age, partnership rates, numbers of children and education). However, the advantage using visual techniques is to allow a richer (and perhaps more realistic) view of cross-national variation that may reveal patterns that are hidden by a simple ‘summary' regression coefficient. As an illustration, the graph distinguishes between countries in North-West Europe, Southern European, Eastern Europe and Scandinavia (Nordic countries). The sample size is small but nevertheless suggestive of some possible clusters, for instance the NW European group tends to work relatively few 
hours and spend relatively little on childcare (except for France) while the Nordic countries have high childcare spending and work relatively long hours (further evidence is provided by estimates based on 26 countries, available from authors on request). Patterns of this sort in the data, together with information about country institutions, may lead to the development of further hypotheses about the determinants of working hours.

\section{Non-linear (binary logit) model}

The estimated parameters for the logit model of work participation are presented in Table 4 and Figure 12. The dependent variable is a binary variable equal to one if a women participates in employment and zero otherwise, with the same explanatory variables as in the linear model. Estimation is based on the previous sample of workers combined with additional observations on non-working women (18-64 years), giving a total sample size of $73,169$.

From the little evidence cited in previous studies and our Monte Carlo simulations we expect similar issues to those encountered with the linear model, except that the biases and excessive non-coverage rates may be worse for small numbers of countries. As before, we take the two-step results as a benchmark: the point estimates and standard errors of the individual-level predictor parameters at step 1 should be accurate thanks to large sample sizes within countries; and the step 2 OLS estimates of the country-level predictor parameter and country intercept variance should be unbiased (with the correct standard error on the countrylevel predictor parameter).

As for the linear model, the fixed parameter estimates from the pooled models (methods 1-2) differ from multilevel estimates (methods 3-6), although by less than in the linear case. ${ }^{27}$ The largest proportionate difference is for the parameter on cohab, which is 0.10 in the pooled model but only 0.07 in the multilevel models. The unclustered standard errors of the individual-level predictor effects are almost identical to those of the multilevel models, but (as in the linear case) the unclustered standard error for the country-level predictor effect is much smaller in the pooled estimates (0.032 compared with 0.284 in the

\footnotetext{
${ }^{27}$ The estimates from the pooled and the multilevel logits are not directly comparable because: (a) the composite error term in the pooled logit, $u_{c}+\varepsilon_{i c}$, is assumed to follow the logistic distribution, while in the random effects logit $u_{c}$ is assumed to be normal and only $\varepsilon_{i c}$ has a logistic distribution; (b) the estimated fixed parameters are scaled by $1 / \operatorname{sd}\left(u_{c}+\varepsilon_{i c}\right)$ in the pooled logit, but only by $1 / \operatorname{sd}\left(\varepsilon_{i c}\right)$ in the multilevel logit. To make the scaling comparable, it is easily shown that the multilevel parameters should be multiplied by $\sqrt{(}(-I C C)$. In our example, $I C C=0.020$ and so the multiplication factor is 0.992 , a trivial adjustment which, nevertheless, increases very slightly the difference between the two sets of estimates.
} 
two-step method). The use of clustering brings the country-level predictor standard error almost up to those of methods 3-6, but (as in the linear model) clustering appears to overstate the standard errors of the individual-level predictor parameters (for example, the standard error of the nownch parameter is 0.045 in the pooled estimates, but only 0.01 in the multilevel estimates). This again suggests that clustered standard errors may lead to misleading conclusions with small numbers of groups.

Focussing on the methods that account for the multilevel data structure, we see that the estimated fixed parameters from the RE and MLM (FML) logits are almost identical to the two-step estimates, consistent with the negligible parameter bias found in the Monte Carlo simulations. The estimates of the FE logit are also the same as the corresponding estimates from the RE logit, indicating (as for the linear model) that the choice between FE and RE is unimportant if interest focuses on the individual-level predictors.

Turning to the standard errors on the estimated fixed parameters, we see that they are identical across methods 3-6 for the individual-level predictors but not for country-level regressor. From the Monte Carlo simulations, we expected the standard error on the chexp parameter to be downward biased by about 15\% (Figure 7). The 'benchmark' standard error from the two-step method is 0.284 , while the RE and MLM standard errors are both 0.255 , implying that they are understated by $10 \%$. This is somewhat less than expected from the Monte Carlos, but in this example we will see that it is enough to change the conclusion about the effect of national childcare spending on work participation.

The corresponding test statistics for the effect of chexp are 0.529/0.255 $=2.07$ from the RE and MLM models, and 0.529/0.284 = 1.86 from the two-step method. If the RE/MLM test statistic is referred to the standard normal distribution, then the $p$-value, as reported by Stata, is 0.038 . Thus the chexp estimate is significant at the $5 \%$ level. By contrast the $p$-value of the two-step test statistic, referred to the $t(8)$-distribution and as reported by Stata, is $0.100{ }^{28}$ Therefore the estimated parameter is not significant at the $5 \%$ level and not quite significant even at the $10 \%$ level. We see how an underestimate of the standard error in the MLM leads to an overly liberal conclusion. A more conservative strategy may be to use the $t$ distribution instead of the normal as a reference for the MLM estimates (Raudenbush and Bryk 2002: 282). Using the $t(8)$-distribution, the $p$-value of the RE/MLM test statistic is 0.072 , so the estimated effect is no longer significant at the $5 \%$ level, although it is still

\footnotetext{
${ }^{28}$ Step 2 of the two-step method is an OLS regression using 10 country observations. The 8 degrees of freedom are equal to 10 observations minus a constant and one country-level regressor.
} 
significant at $10 \%$. However, since most statistical software reports p-values based on an asymptotic normal distribution (an exception is HLM), this correction needs to be done manually by users.

Lastly, we compare the standard deviation of the random intercept, as estimated by the RE and MLM methods and the two-step method. From the Monte Carlos simulations, we expect sig_u to be downward biased by about 15\%. The gap in the estimates is somewhat less but nevertheless quite substantial: the RE and MLM estimates (0.229) are $11 \%$ less than the two-step estimate (0.258). The difference leads to a correspondingly lower value of ICC in RE/MLM (0.016) than two-step (0.020).

For a graphical view of country-level variation, the country-specific intercepts are plotted against national childcare expenditure in Figure 12. There is a clearer upward slope than for hours worked, suggesting that more childcare spending may be associated with greater work participation, although the data point for Denmark may be exerting undue influence. There is somewhat less evidence of distinct country clusters than in the working hours model, although Finland and Denmark have high participation rates (also high levels of childcare spending).

\section{Summary and conclusions}

When there are few countries in a multi-country data set, there is little information with which to estimate country effects, whether these effects refer to the fixed parameters on country-level predictors or the variances of random country intercepts. Multilevel model users need to be cautious in the claims they make about country effects.

Our Monte-Carlo simulations suggest that users require at least 25 countries for linear models and at least 30 countries for logit models. With fewer countries, estimates of countrylevel fixed parameters are likely to be estimated imprecisely and this will not be adequately reflected in test statistics reported by commonly-used software: users will conclude too often that a country effect exists when it does not. Country random variances will be biased downwards and have confidence intervals that are too narrow. The only estimates that are unaffected by the small number of countries are the fixed parameters on individual-level predictors (the number of individuals per country is typically large): provided there is not also a random component attached to the slope, these parameters are estimated without bias and with the correct standard errors (and non-coverage rate). 
Since the critical number of countries required for reliable estimation of country effects is larger than is available in many existing datasets, what can analysts do in the small- $C$ case? We recommend three approaches. One is to supplement regression-based modelling with more descriptive analysis of measured country differences. We have referred to exploratory data analysis, including graphical representations of country differences, which may reveal features of the data (including outliers and country groupings) that are hidden when fitting a simple regression line. A second approach is to explore methods that are more robust to small numbers of countries. These include the two-step method, small sample corrections to test statistics, and bootstrapping; although some of these techniques require specialised knowledge and are available in only a few software packages. A third approach would be to move beyond classical (frequentist) statistics and make greater use of Bayesian methods of estimation and inference, as they appear to perform better in the small- $C$ case. The problem is that these methods also require statistical expertise beyond that of most applied social science researchers, as well as specialist software. With any of these approaches, the need for detailed consideration of the workings of national institutions and policies remains.

\section{References}

Aachen, C. H. (2005). 'Two-step hierarchical estimation: beyond regression analysis’, Political Analysis, 13, 447-456.

Angrist, J. D. and Pischke, J.-S. (2009). Mostly Harmless Econometrics: An Empiricist's Companion. Princeton NJ: Princeton University Press.

Austin, P. C. (2010). 'Estimating multilevel logistic regression models when the number of clusters is low: a comparison of different statistical software procedures', International Journal of Biostatistics, 6, article 16.

Beck, N. and Katz, J. N. (1995). 'What to do (and not to do) with time-series cross-section data', American Political Science Review, 89, 634-647.

Bell, R. M. and McCaffrey, D. F. (2002). 'Bias reduction in standard errors for linear regression with multistage samples’, Survey Methodology, 28, 169-181.

Bester, C. A., Conley, T. G., Hansen, C. B. (2011). 'Inference with dependent data using cluster covariance estimators', Journal of Econometrics, 165, 137-151.

Borjas, G. J. and Sueyoshi, G. T. (1994). 'A two-stage estimator for probit models with structural group effects', Journal of Econometrics, 64,165-182. 
Bowers, J. and Drake, K. W. (2005). 'EDA for HLM: visualization when probabilistic inference fails’, Political Analysis, 13, 301-326.

Brewer, M., Crossley, T. F., and Joyce, R. (2013). 'Inference with Difference-in-Differences Revisited'. Paper for the $8^{\text {th }}$ IZA Conference on Labor Market Evaluation, London. http://www.iza.org/conference_files/PolicyEval_2013/joyce_r8616.pdf

Browne, W. C. and Draper, D. (2000). 'Implementation and performance issues in Baysian and likelihood fitting of multilevel models’, Computational Statistics, 15, 391-420.

Browne, W. J., and Draper, D. (2006). 'A comparison of Bayesian and likelihood-based methods for fitting multilevel models', Bayesian Analysis, 1, 473-514.

Cameron, C. A., Gelbach, J. B., and Miller, D. L. (2008). 'Bootstrap-based improvements for inference with clustered standard errors', Review of Economics and Statistics, 90, 414-427.

Cameron, C. A., and Trivedi P. K. (2010), Microeconometrics using Stata, revised edition. College Station TX: Stata Press.

Card, D. (1995). 'The wage curve: a review’, Journal of Economic Literature, 33, 285-299.

Carpenter, J. R., Goldstein, H., and Rasbash, J. (2003). ‘A novel bootstrap procedure for assessing the relationship between class size and achievement', Journal of the Royal Statistical Society, Series C, 52, 431-443.

DiPrete, T. A. and Forristal, J. D. (1994). 'Multilevel models: methods and substance', Annual Review of Sociology, 20, 331-357.

Donald, S. G. and Lang, K. (2007). 'Inference with difference-in-differences and other panel data', Review of Economics and Statistics, 89, 221-233.

Gelman, A. (2006). 'Prior distributions for variance parameters in hierarchical models', Bayesian Analysis, 1, 515-533.

Greene, W. (2004). 'The behaviour of the maximum likelihood estimator of limited dependent variable models in the presence of fixed effects', Econometrics Journal, 7, 98-119.

Hanushek, E. A. (1974). 'Efficient estimators for regressing regression coefficients', American Statistician, 28, 66-67.

Hox, J. J. (2010), Multilevel Analysis: Techniques and Applications, 2nd edition. London: Routledge.

Hsiao, C. (2003). Analysis of Panel Data, 2nd edition. Cambridge: Cambridge University Press. 
Huber, J. D., Kernell, G., and Leoni, E. (2005). 'Institutional context, cognitive resources and partisan attachments across democracies’, Political Analysis, 13, 365-386.

Jusko, K. L., and Shively, W. P. (2005). 'A two-step strategy for the analysis of crossnational public opinion data', Political Analysis, 13, 327-344.

Kedar, O. (2005). 'How diffusion of power in parliaments affects voter choice', Political Analysis, 13, 410-429.

Kedar, O. and Shively, W. P. (2005). 'Introduction to the special issue', Political Analysis, 13, 297-300.

Kenward, M. G and Roger, J. H. (1997). 'Small sample inference for fixed effects from restricted maximum likelihood', Biometrics, 53, 983-997.

Maas, C. J. M. and Hox, J. J. (2004). 'Robustness issues in multilevel regression analysis', Statistica Neerlandica, 58, 127-137.

Maas, C. J. M. and Hox, J. J. (2005). 'Sufficient sample sizes for multilevel modeling', Methodology, 1, 86-92.

Moineddin, R., Matheson, F. I., and Glazier, R. H. (2007). 'A simulation study of sample size in multilevel regression models’, BMC Medical Research Methodology, 7: article 34.

Moulton, B. R. (1986). 'Random group effects and the precision of regression estimates,' Journal of Econometrics, 32, 385-397.

Pinheiro, J. C. and Chao, E. C. (2006). 'Efficient Laplacian and adaptive quadrature algorithms for multilevel generalized linear mixed models', Journal of Computational and Graphical Statistics, 15, 58-81.

Raudenbush, S. W. and Bryk, A. S. (2002). Hierarchical Linear Models: Applications and Data Analysis Methods, Second Edition. Thousand Oaks CA: Sage Publications.

Raudenbush S. W., Bryk, A. S., Cheong, Y. F., and Congdon, R. T. Jr. (2004). HLM 6: Hierarchical Linear and Nonlinear Modeling. Lincolnwood IL: Scientific Software International.

Rodriguez, G. and Goldman, N. (1995). 'An assessment of estimation procedures for multilevel models with binary responses', Journal of the Royal Statistical Society, Series A, 158, 73-89.

Rodriguez, G. and Goldman, N. (2001). 'Improved estimation procedures for multilevel models with binary response: case study', Journal of the Royal Statistical Society, Series A, 164, 339-355.

Saxonhouse, G. R. (1976). 'Estimated parameters as dependent variables', American Economic Review, 66, 178-183. 
Snijders, T. A.B. and Bosker, R. (1999). Multilevel Analysis: An Introduction to Basic and Advanced Multilevel Modeling. Thousand Oaks CA: Sage Publications Ltd.

StataCorp (2011). Stata Statistical Software, Release 12. College Station, TX: StataCorp.

Stegmueller, D. (2013). 'How many countries do you need for multilevel modeling? A comparison of Bayesian and frequentist approaches', American Journal of Political Science, 57, 748-761.

Wang,J., Carpenter, J. R., and Kepler, M. A. (2006). 'Using SAS to conduct nonparametric residual bootstrap multilevel modeling with a small number of groups', Computer Methods and Programs in Biomedicine, 82, 130-143. With Corrigendum, 85(2007) 185-186.

Wooldridge, J. (2010). Econometric Analysis of Cross Section and Panel Data, 2nd edition. Cambridge MA: MIT Press. 
Table 1. Multi-country datasets commonly-used in social science research

\begin{tabular}{lc}
\hline Data sources (in alphabetical order) & $\begin{array}{c}\text { Typical number of } \\
\text { countries per wave } \\
\text { of data }\end{array}$ \\
\hline Eurobarometer & 27 \\
European Community Household Panel (ECHP) & 15 \\
European Quality of Life Survey (EQLS) & 31 \\
European Social Survey (ESS) & 30 \\
European Union Statistics on Income and Living Conditions (EU-SILC) & 27 \\
European Values Study (EVS) & 45 \\
International Social Survey Program (ISSP) & 36 \\
Luxembourg Income Study (LIS) & 32 \\
Survey of Health, Ageing and Retirement in Europe (SHARE) & 14 \\
\hline Notes: All datasets are based on cross-sectional surveys with the exception of ECHP and SHARE which are panel \\
surveys. EU-SILC has cross-sectional and panel components; and data collection is via administrative registers rather \\
than household surveys for some countries.
\end{tabular}

Table 2: Model specifications and parameter values for simulation analysis

\begin{tabular}{llrrrr}
\hline Regressors & Parameter & \multicolumn{4}{c}{ Parameter values } \\
& label & \multicolumn{3}{c}{ Hours } & \multicolumn{2}{c}{ Participation } \\
& & Basic & Extended & Basic & Extended \\
\hline Fixed effects & & & & & \\
constant & b0 & 22 & 22 & -9.1 & -9.1 \\
age $_{i c}$ & b1 & 0.8 & 0.8 & 0.5 & 0.5 \\
(age $\left._{i c}\right)^{2}$ & b2 & -0.01 & -0.01 & -0.006 & -0.006 \\
cohab $_{i c}$ & b3 & -1 & -1 & 0.02 & 0.02 \\
nownch $_{i c}$ & b4 & -1.2 & -1.2 & -0.27 & -0.27 \\
isced3 $_{i c}$ & b5 & 0.7 & 0.7 & 0.7 & 0.7 \\
isced4 $_{i c}$ & b6 & 1.4 & 1.4 & 0.9 & 0.9 \\
isced56 $_{i c}$ & b7 & 1.6 & 1.6 & 1.4 & 1.4 \\
chexp $_{c}$ & c1 & -0.23 & -2.7 & 0.98 & 0.7 \\
chexp $_{c} \times$ cohab $_{i c}$ & c2 & & 2.4 & & 0.6 \\
chexp $_{c} \times$ nownch $_{i c}$ & c3 & & 0.7 & & -0.1 \\
Random effects & & & & & \\
$\sigma_{e}$ & sig_e & 9.5 & 9.4 & $\pi / \sqrt{ } 3$ & $\pi / \sqrt{ } 3$ \\
$\sigma_{u}$ & sig_u & 3.5 & 2.4 & 0.275 & 0.38 \\
$\sigma_{b 3 c}$ & sig_b3c & & 1.2 & & 0.25 \\
$\sigma_{b 4 c}$ & sig_b4c & & 1.2 & & 0.13 \\
ICC & & 0.120 & 0.061 & 0.022 & 0.042 \\
\hline
\end{tabular}

Notes. See main text for explanation of the models and regressors. The random effects are: an individual-specific error $e_{i c} \sim \mathrm{N}\left(0, \sigma_{e}^{2}\right)$; a random intercept $u_{c} \sim \mathrm{N}\left(0, \sigma_{u}^{2}\right)$; a random coefficient on cohab ${ }_{i c}, b 3 c \sim \mathrm{N}\left(0, \sigma_{b 3 c}{ }^{2}\right)$; and a random coefficient on nownch ${ }_{i c}$, $b 4 c \sim \mathrm{N}\left(0, \sigma_{b 4 c}{ }^{2}\right)$. chexp $\mathrm{p}_{c}$ is the country-level regressor. 
Table 3. Model of working hours with country-specific intercepts: alternative estimation methods compared

\begin{tabular}{|c|c|c|c|c|c|c|}
\hline \multirow[b]{2}{*}{ Method } & \multicolumn{6}{|c|}{ Parameter estimates (standard errors) } \\
\hline & $\operatorname{cohab}_{i c}$ & nownch $_{i c}$ & chexp $_{c}$ & sig_u & sig_e & ICC \\
\hline \multirow[t]{2}{*}{ 1. OLS } & $-1.651^{* * *}$ & $-1.177 * * *$ & $1.876^{* * *}$ & & & \\
\hline & $(0.125)$ & $(0.055)$ & $(0.184)$ & & & \\
\hline \multirow[t]{2}{*}{ 2. OLS (clust SE) } & $-1.651^{*}$ & $-1.177 *$ & 1.876 & & & \\
\hline & $(0.836)$ & $(0.586)$ & $(4.351)$ & & & \\
\hline \multirow[t]{2}{*}{ 3. FE } & $-1.151^{* * *}$ & $-1.585 * * *$ & & & 9.881 & \\
\hline & $(0.114)$ & $(0.051)$ & & & & \\
\hline \multirow[t]{2}{*}{ 4. RE (GLS ) } & $-1.152^{* * *}$ & $-1.585 * * *$ & 0.423 & 4.872 & 9.881 & 0.196 \\
\hline & $(0.114)$ & $(0.051)$ & $(5.370)$ & & & \\
\hline \multirow[t]{2}{*}{ 5. MLM (REML) } & $-1.152^{* * *}$ & $-1.585^{* * *}$ & 0.424 & $4.341^{* * *}$ & $9.880 * * *$ & 0.162 \\
\hline & $(0.114)$ & $(0.051)$ & $(4.785)$ & $(0.972)$ & $(0.033)$ & \\
\hline \multirow[t]{2}{*}{ 6. MLM (FML) } & $-1.152^{* * *}$ & $-1.585^{* * *}$ & 0.424 & $4.341^{* * *}$ & $9.880 * * *$ & 0.162 \\
\hline & $(0.114)$ & $(0.051)$ & (4.785) & $(0.972)$ & $(0.033)$ & \\
\hline \multirow[t]{2}{*}{ 7a. Step 1 (FE) } & $-1.151^{* * *}$ & $-1.585^{* * *}$ & & & 9.881 & \\
\hline & $(0.114)$ & $(0.051)$ & & & & \\
\hline \multirow[t]{2}{*}{ 7b. Step 2 (OLS) } & & & 0.421 & 4.856 & & 0.195 \\
\hline & & & (5.349) & & & \\
\hline
\end{tabular}

Table 4. Model of work participation with country-specific intercepts: alternative estimation methods compared

\begin{tabular}{|c|c|c|c|c|c|}
\hline \multirow[b]{2}{*}{ Method } & \multicolumn{5}{|c|}{ Parameter estimates (standard errors) } \\
\hline & $\operatorname{cohab}_{i c}$ & nownch $_{\text {ic }}$ & chexp $_{c}$ & sig_u & ICC \\
\hline \multirow[t]{2}{*}{ 1. Pooled logit } & $0.100 * * *$ & $-0.294 * * *$ & $0.583^{* * *}$ & & \\
\hline & $(0.021)$ & $(0.009)$ & $(0.032)$ & & \\
\hline \multirow[t]{2}{*}{ 2. Pooled logit (clust SE) } & $0.100 *$ & $-0.294^{* * *}$ & $0.583^{* *}$ & & \\
\hline & $(0.059)$ & $(0.045)$ & $(0.252)$ & & \\
\hline \multirow[t]{2}{*}{ 3. FE logit } & $0.071^{* * *}$ & $-0.288 * * *$ & & & \\
\hline & $(0.021)$ & $(0.010)$ & & & \\
\hline \multirow[t]{2}{*}{ 4. RE logit } & $0.072 * * *$ & $-0.288 * * *$ & $0.529 * *$ & $0.229 * * *$ & 0.016 \\
\hline & $(0.021)$ & $(0.010)$ & $(0.255)$ & $(0.052)$ & \\
\hline \multirow[t]{2}{*}{ 5. MLM logit (FML) } & $0.072 * * *$ & $-0.288 * * *$ & $0.529 * *$ & $0.229 * * *$ & 0.016 \\
\hline & $(0.021)$ & $(0.010)$ & $(0.255)$ & $(0.052)$ & \\
\hline \multirow[t]{2}{*}{ 6a. Step 1} & $0.071^{* * *}$ & $-0.288 * * *$ & & & \\
\hline & $(0.021)$ & $(0.010)$ & & & \\
\hline \multirow[t]{2}{*}{ 6b. Step 2 (OLS) } & & & 0.529 & 0.258 & 0.020 \\
\hline & & & $(0.284)$ & & \\
\hline
\end{tabular}

Notes: other explanatory variables are: age, age squared, and highest education level (3 dummy variables); number of observations is 73,169 and number of countries is 10 ; * significant at $10 \%$; ** significant at $5 \%$; *** significant at 1\%; for methods 1-6a, significance levels are as reported by Stata, and refer to critical values from $z$-distribution; for method $6 \mathrm{~b}$, significance levels refer to critical values from $t(8)$-distribution. 
Figure 1. Relative parameter bias (\%): linear model with random intercept and country-level regressor (Basic model for hours), selected parameters
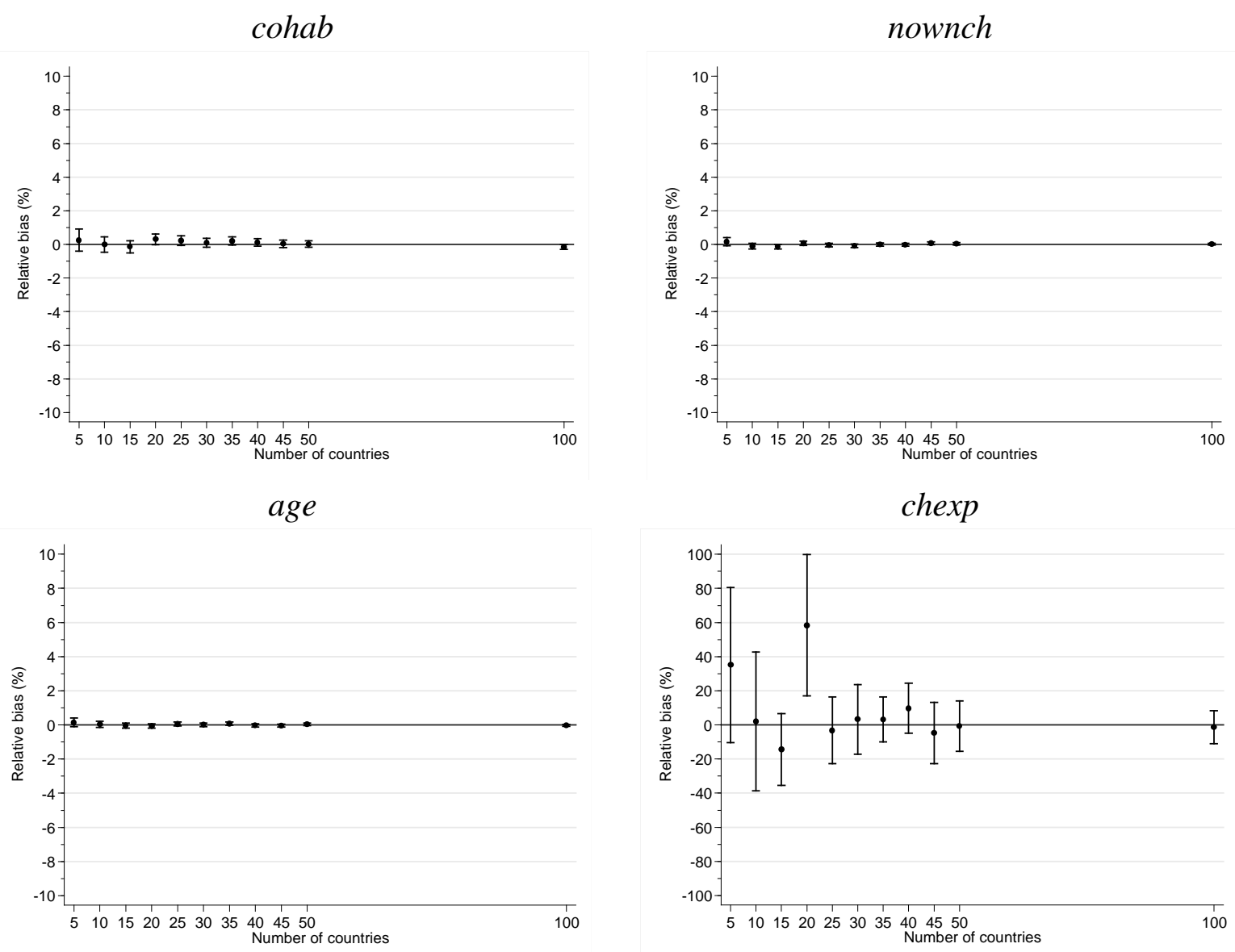

sig_e

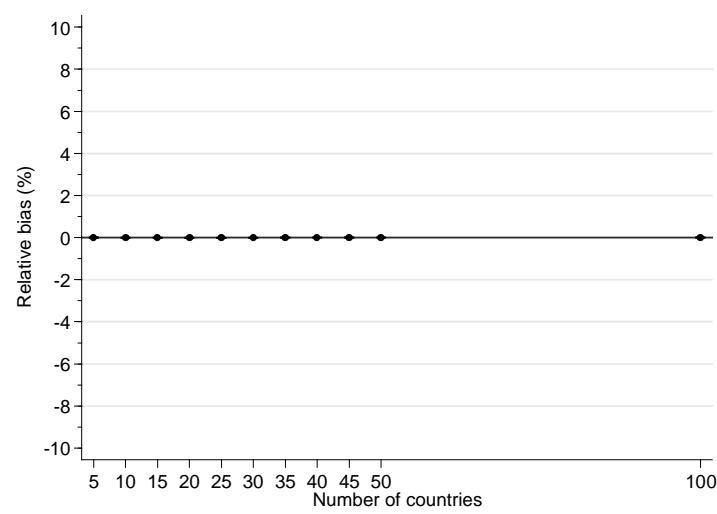

sig_u

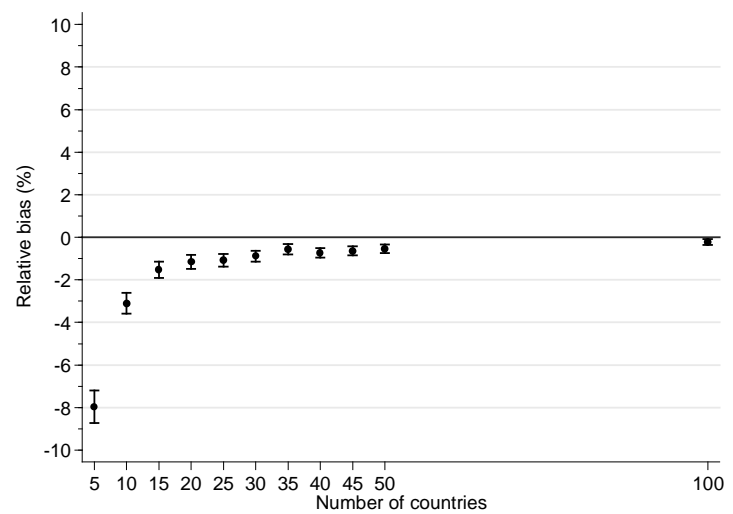


Figure 2. Relative standard error bias: linear model with random intercept and country-level regressor (Basic model for hours), selected parameters

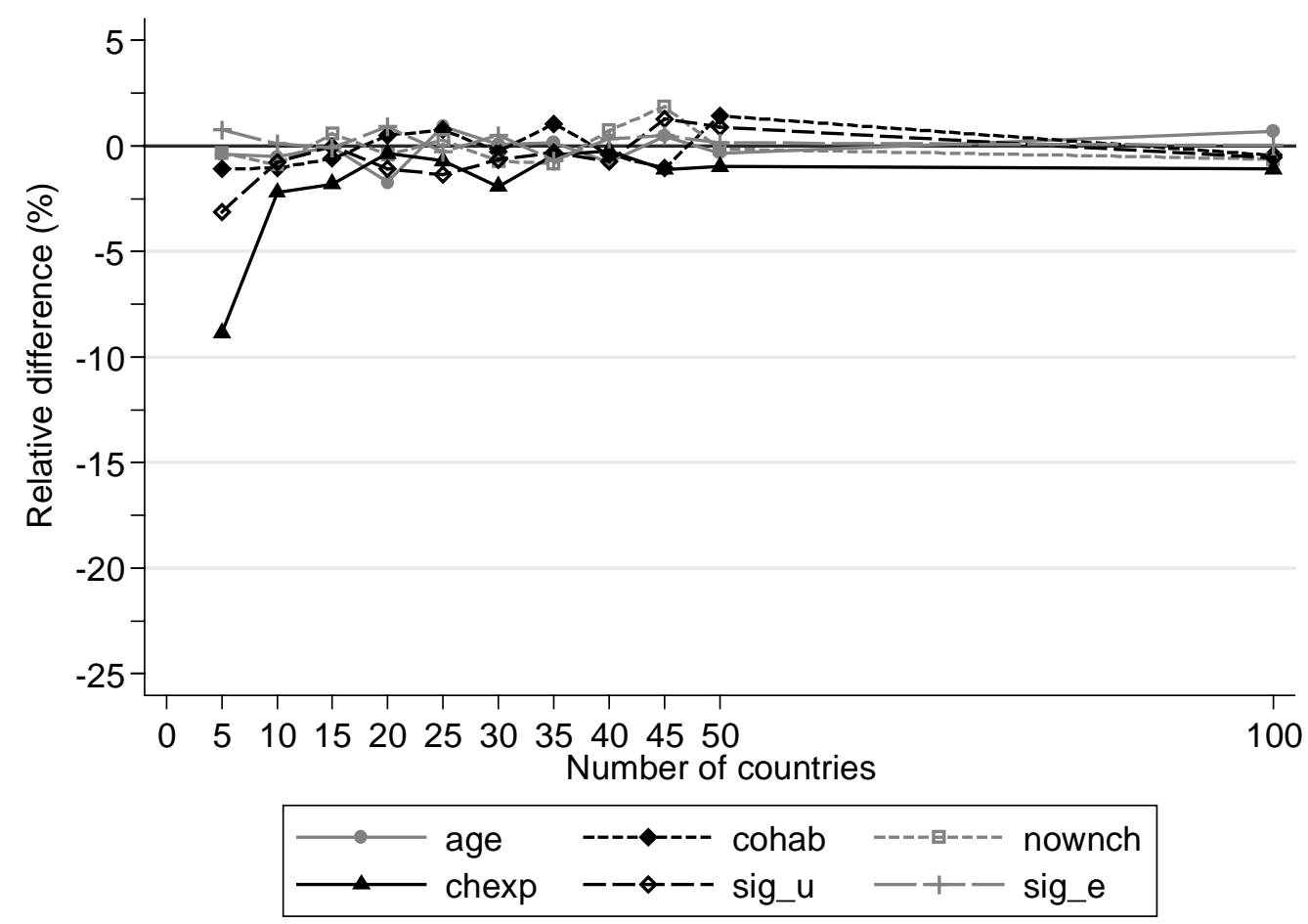


Figure 3. Non-coverage rate: linear model with random intercept and country-level regressor (Basic model for hours), selected parameters

cohab

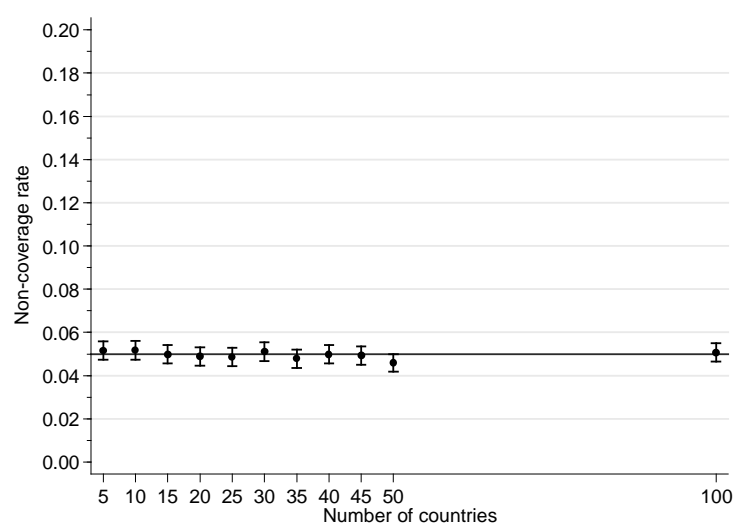

age

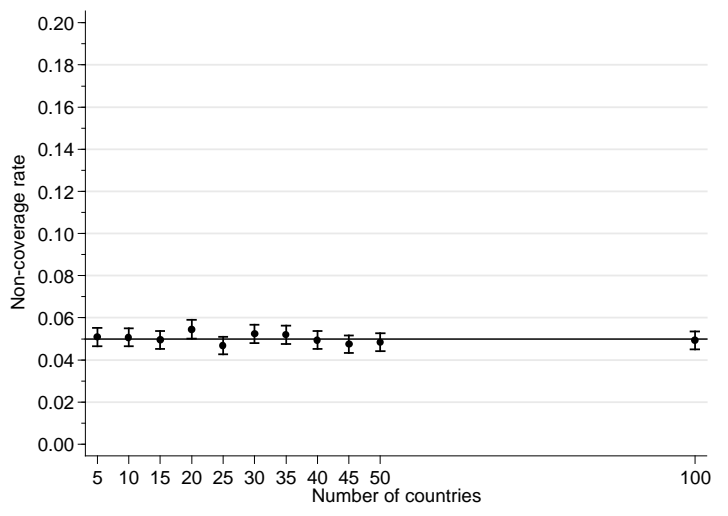

sig_e

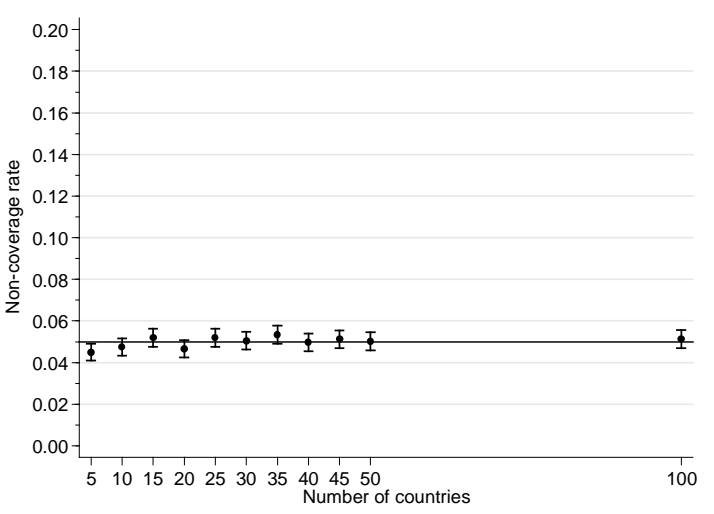

nownch

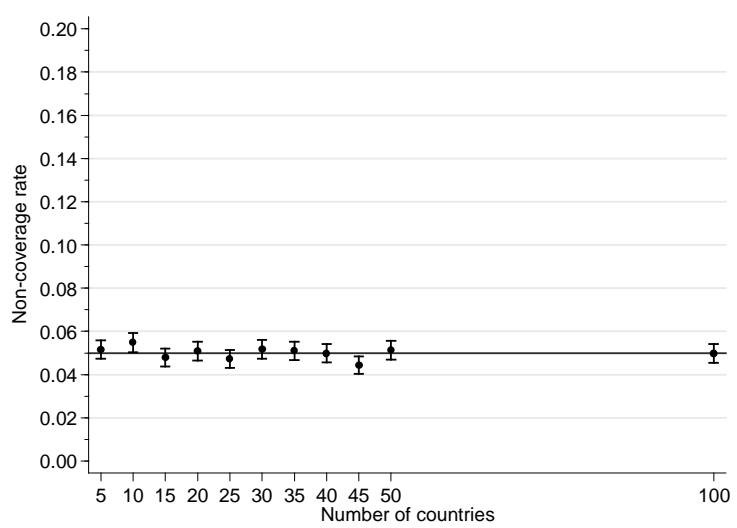

chexp

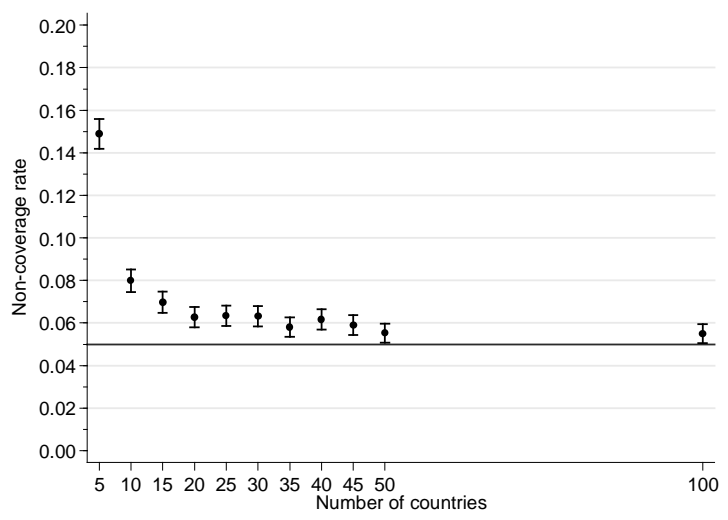

sig_u

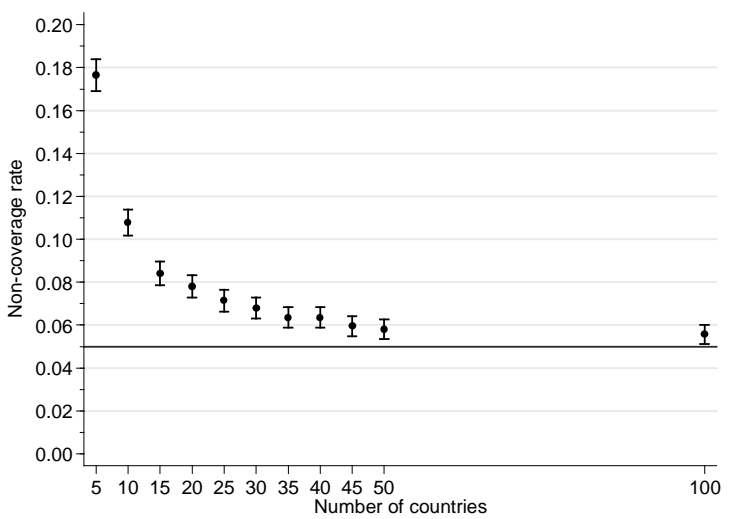


Figure 4. Relative parameter bias (\%): linear model with random intercept, two random slopes, country-level regressor and individual-country interaction (Extended model for hours), selected parameters

cohab

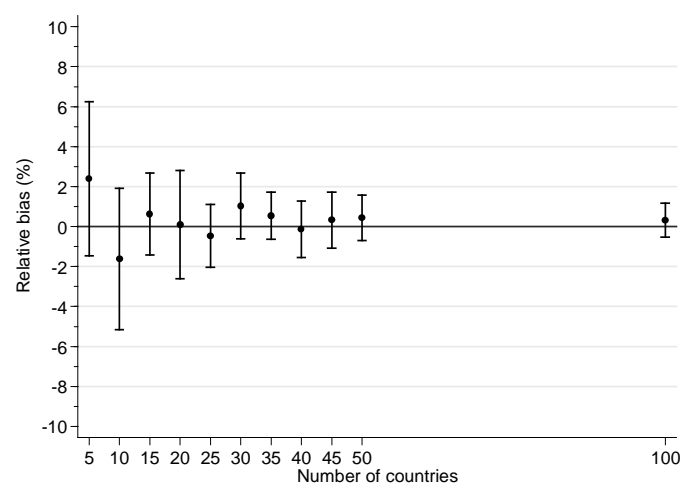

age
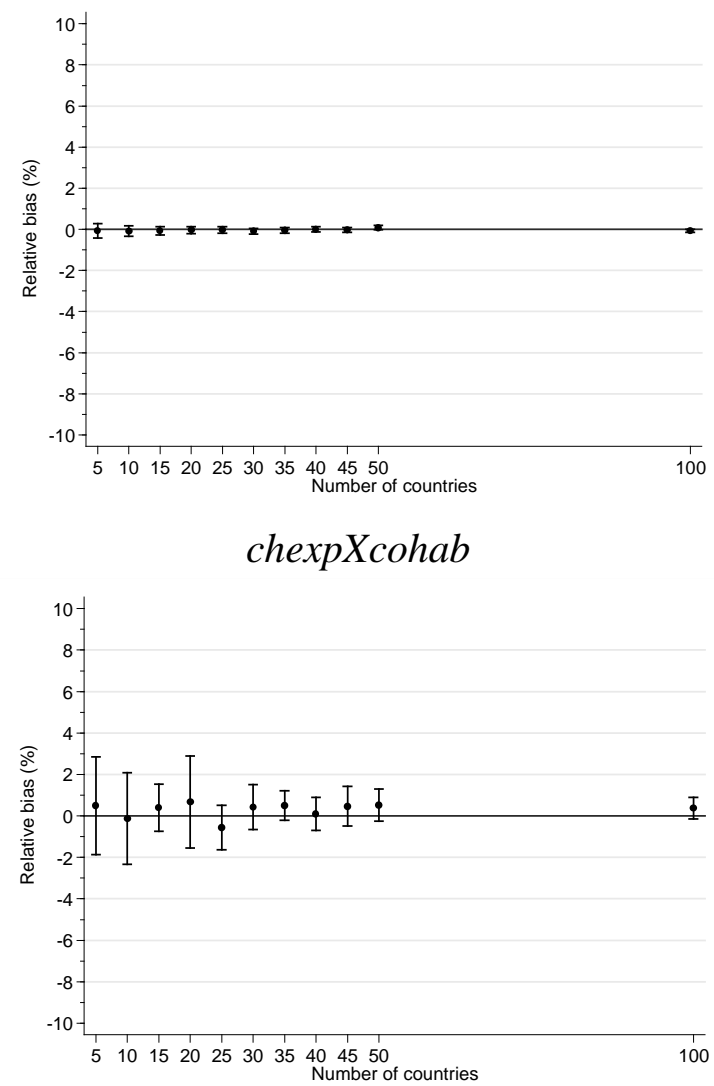

nownch
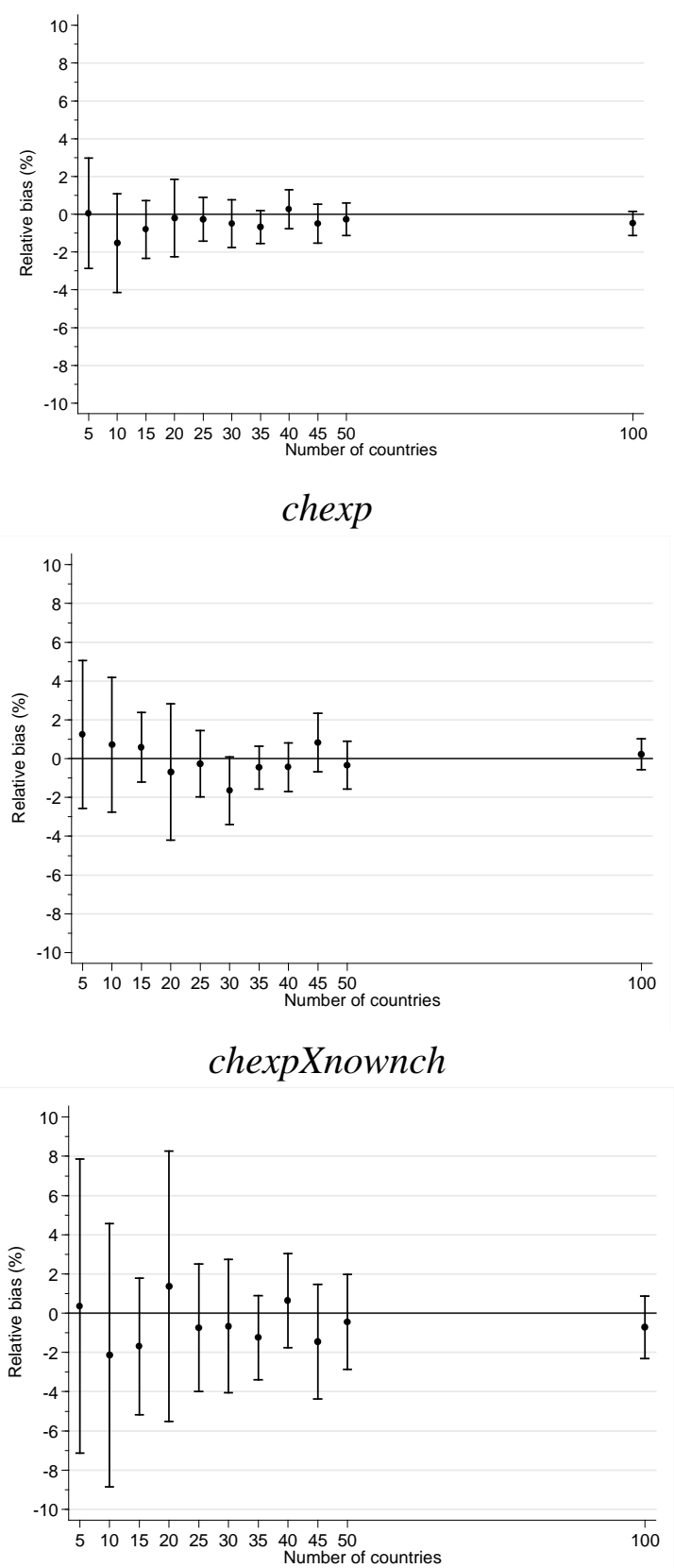

Continued overleaf 
Figure 4 (continued). Relative parameter bias (\%): model with random intercept, two random slopes, country-level regressor and individual-country interaction (Extended model for hours), selected parameters

sig_b3c

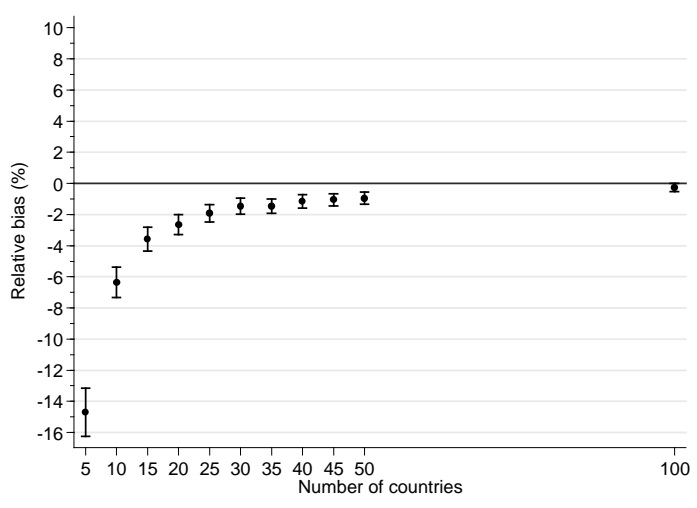

sig_e

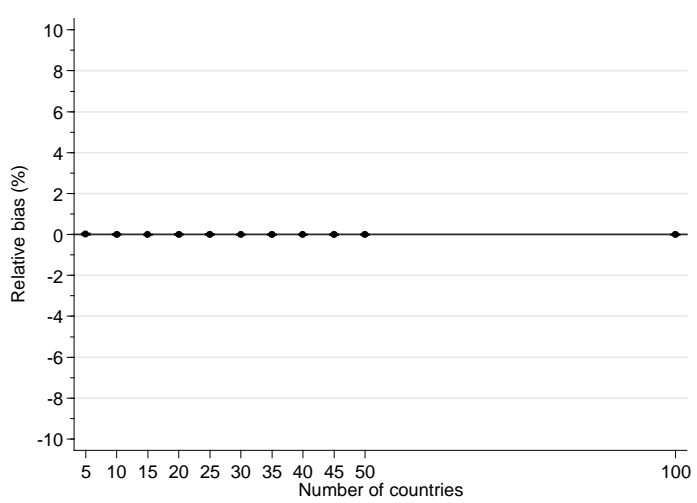

sig_b4c

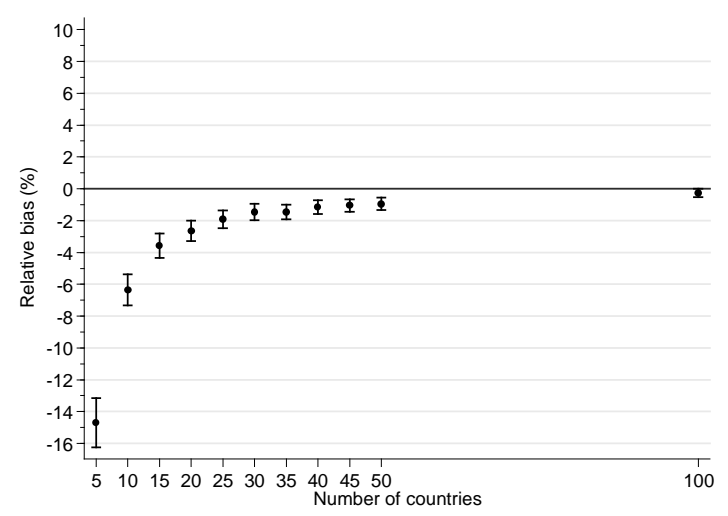

sig_u

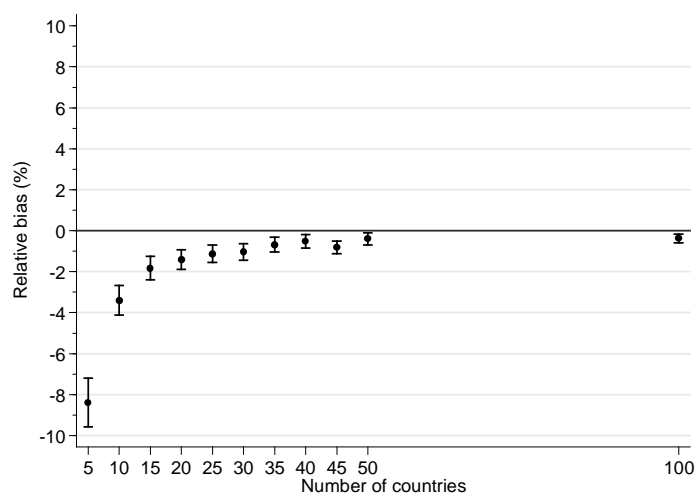


Figure 5. Non-coverage rate: model with random intercept, two random slopes, country-level regressor and individual-country interaction (Extended model for hours), selected parameters

cohab

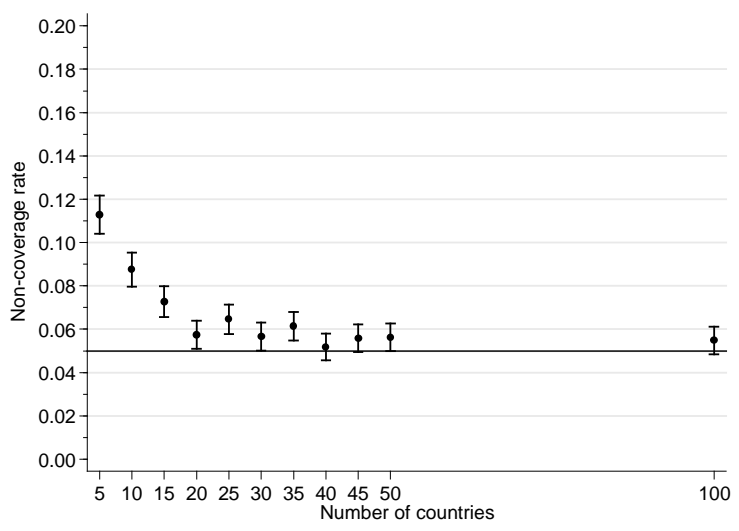

age

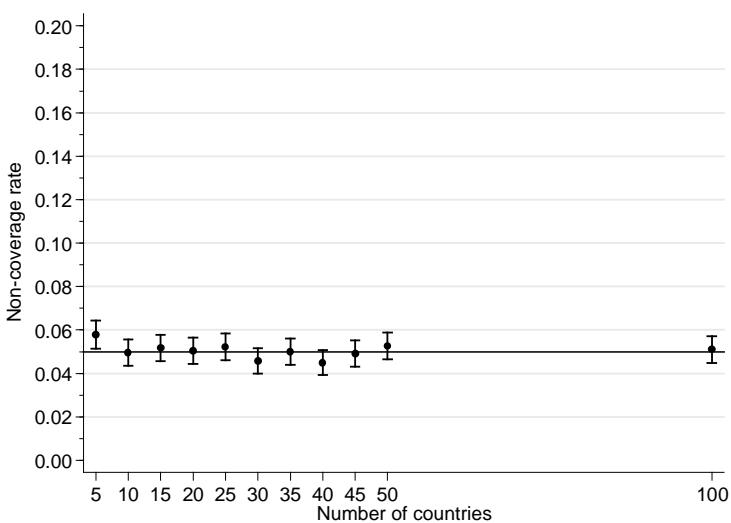

chexpXcohab

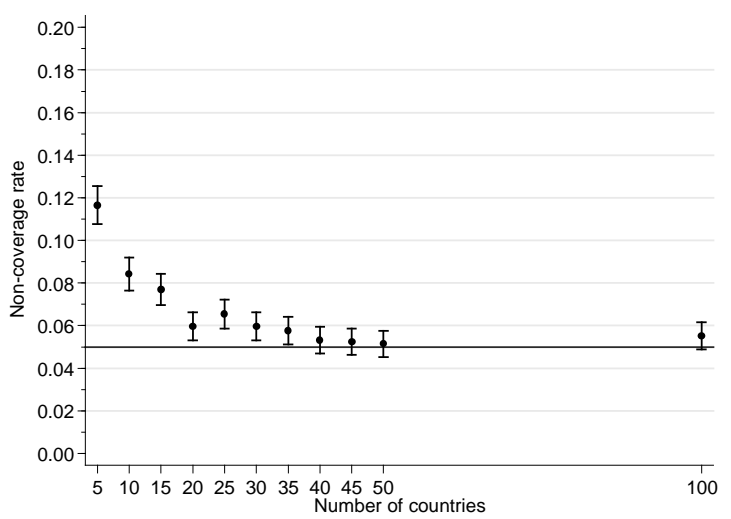

nownch

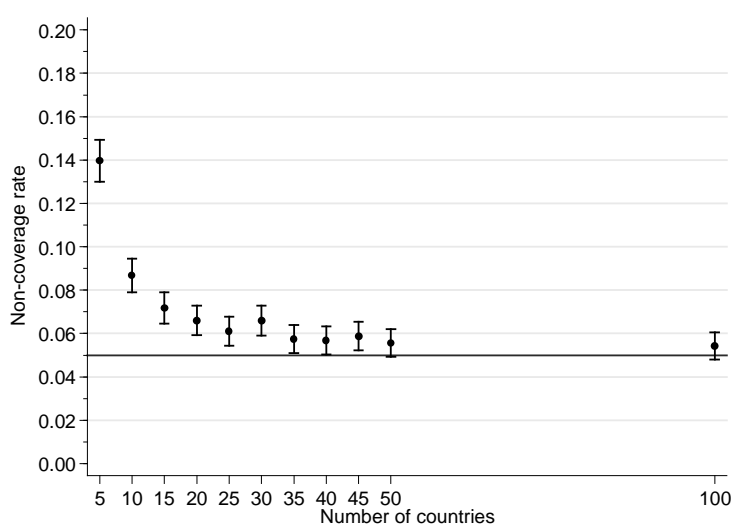

chexp

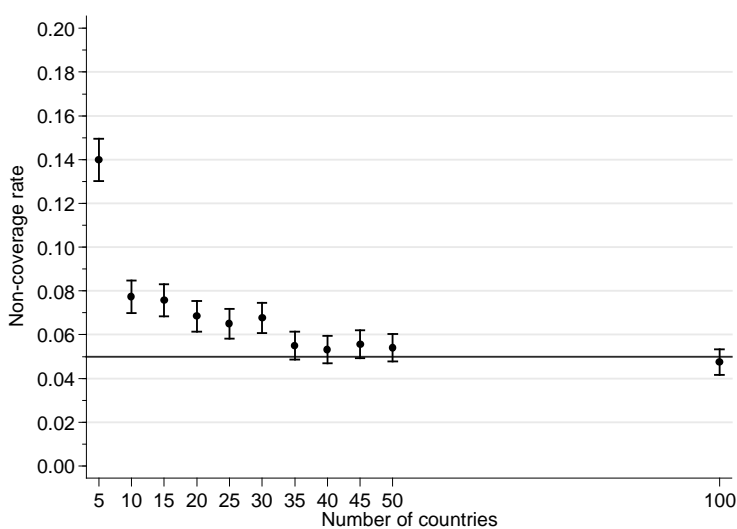

chexpXnownch

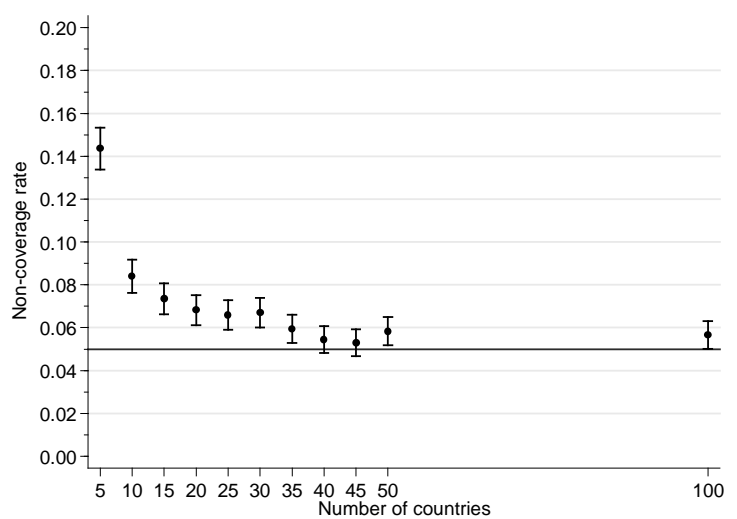

Continued overleaf 
Figure 5 (continued). Non-coverage rate: linear model with random intercept, two random slopes, country-level regressor and individual-country interaction (Extended model for hours), selected parameters

sig_b3c

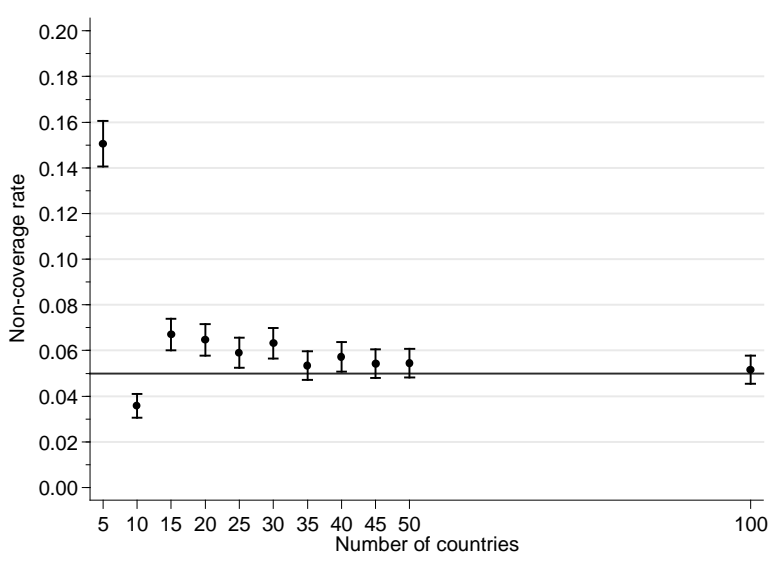

sig_b4c

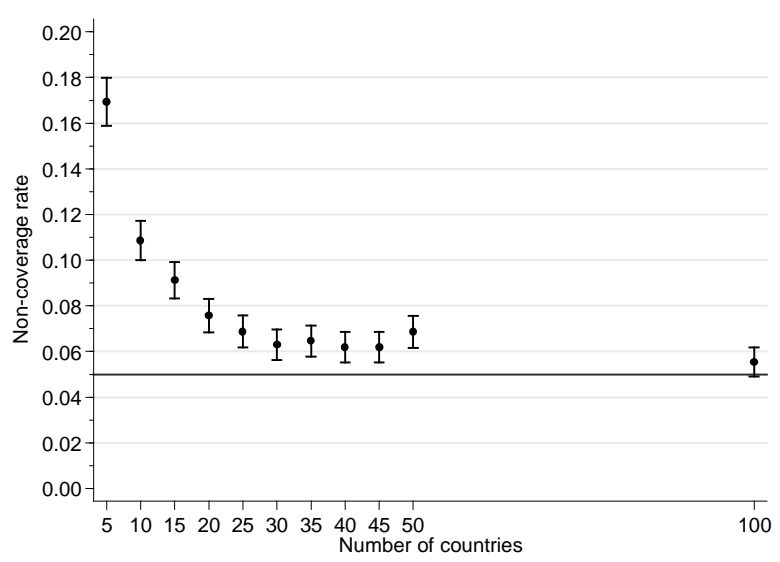

sig_u

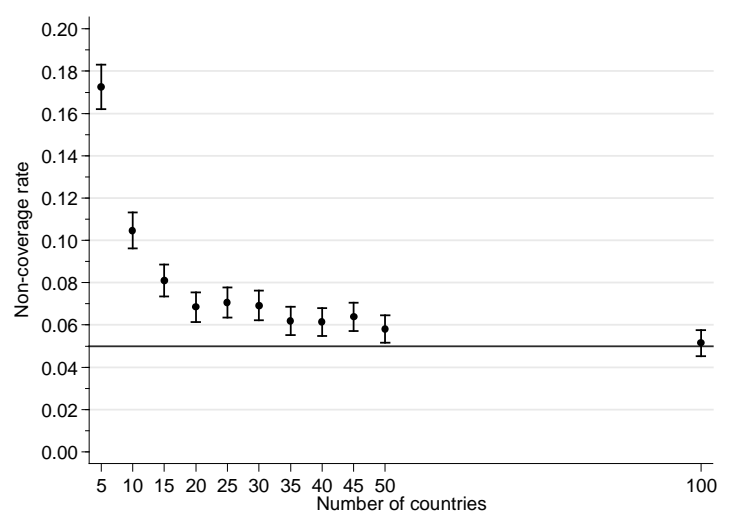


Figure 6. Relative parameter bias (\%): binary logit model with random intercept and countrylevel regressor (Basic model for participation), selected parameters

cohab

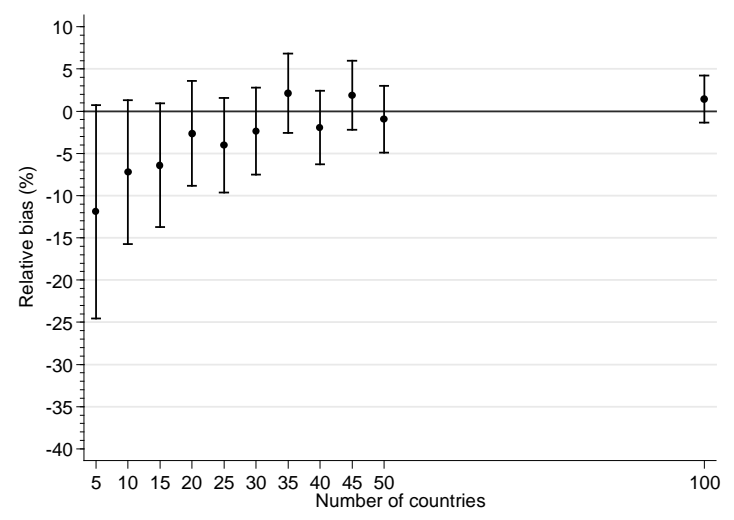

age

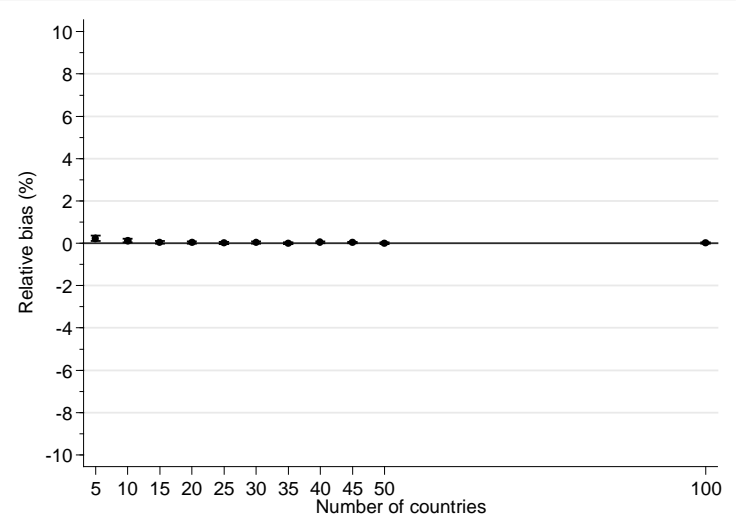

nownch

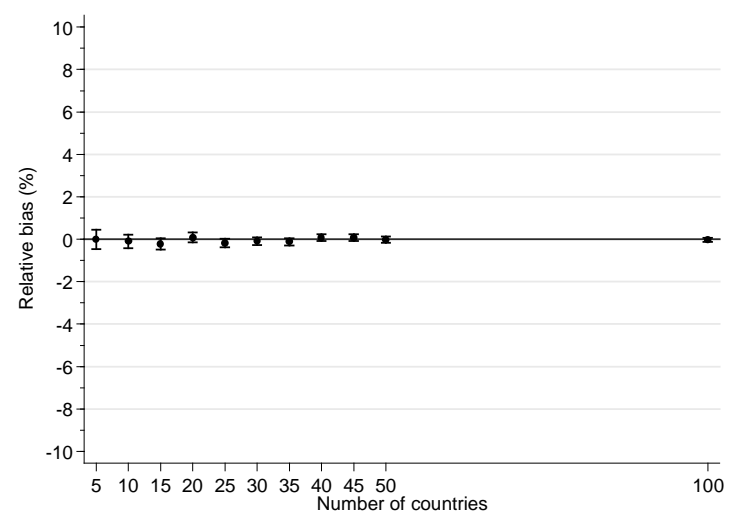

chexp

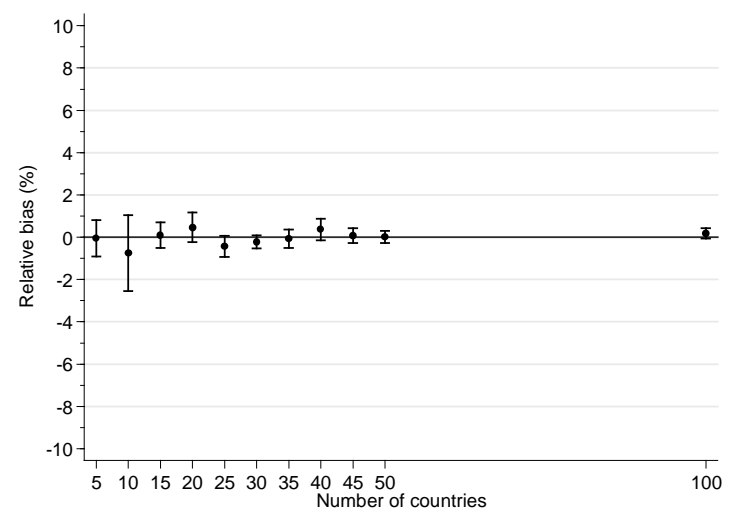

sig_u

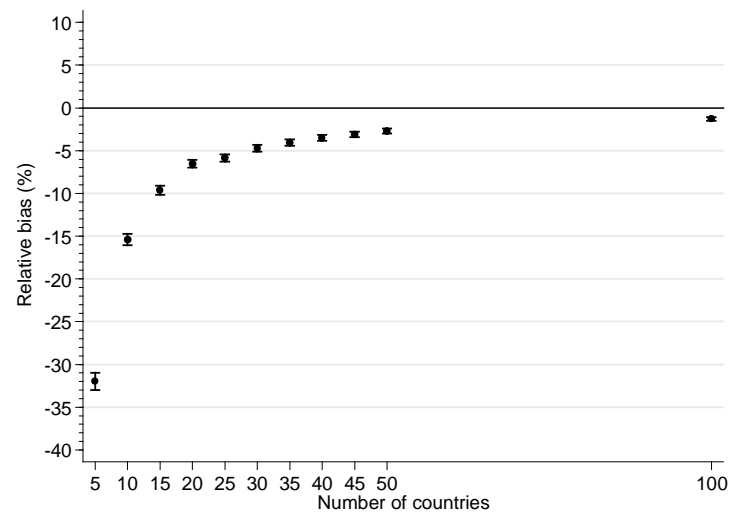


Figure 7. Relative standard error bias: binary logit model with random intercept and countrylevel regressor (Basic model for participation), selected parameters

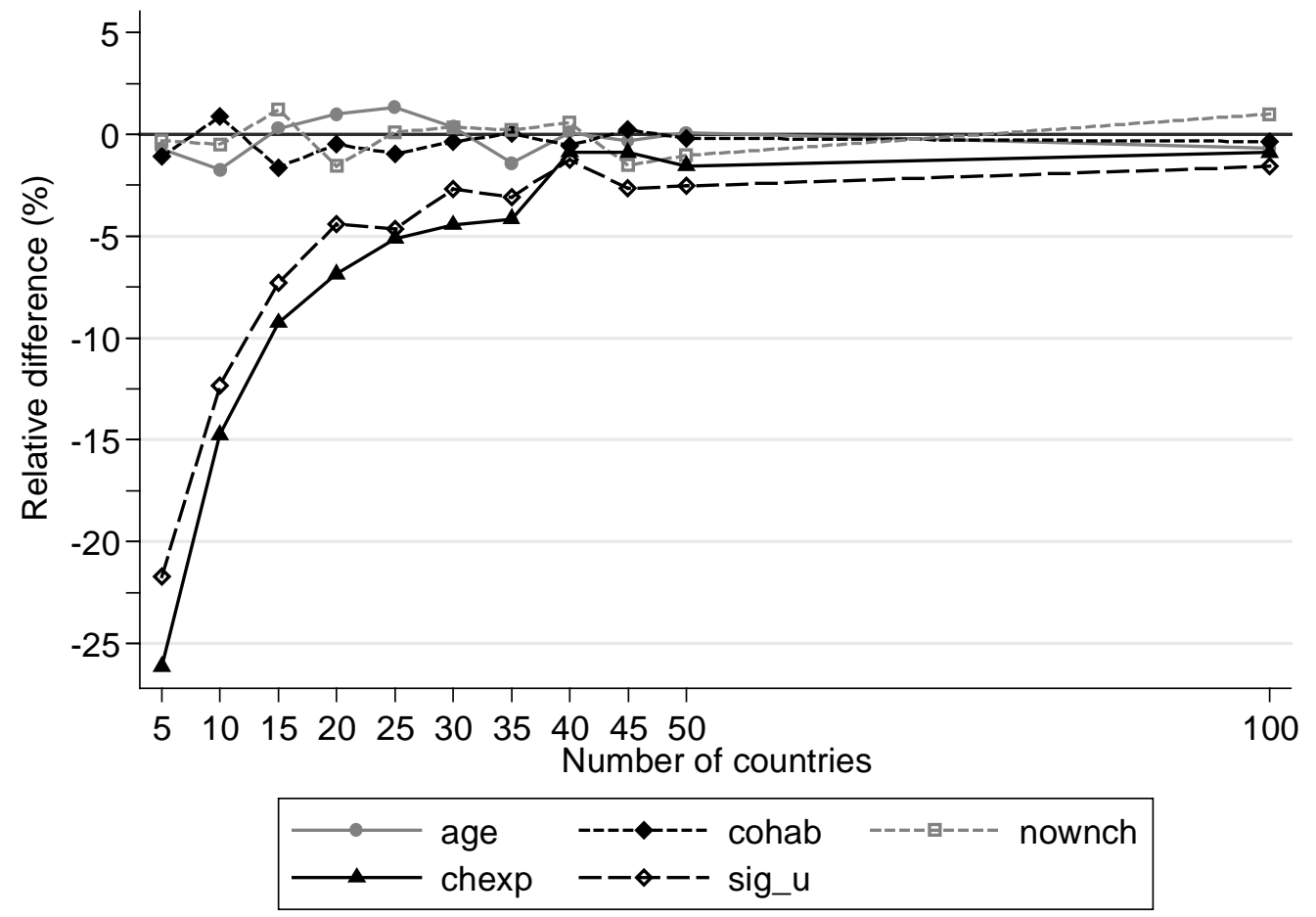


Figure 8. Non-coverage rate: binary logit model with random intercept and country-level regressor (Basic model for participation), selected parameters

cohab

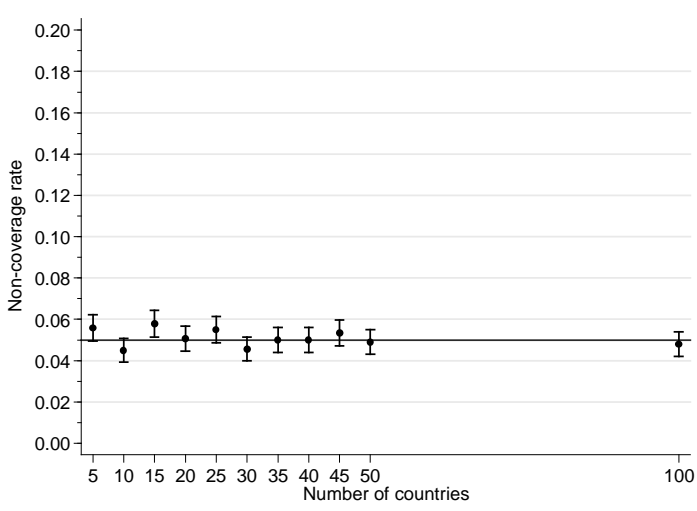

age

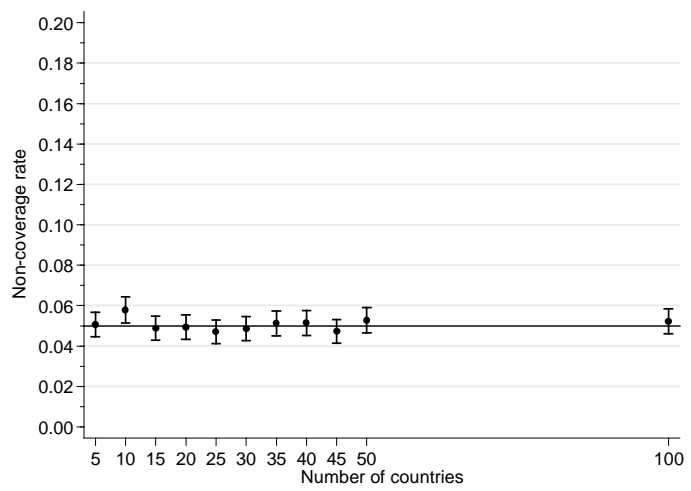

nownch

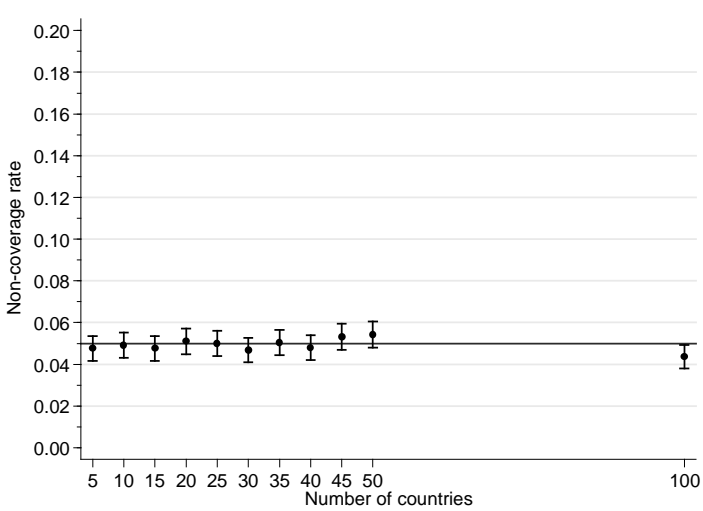

chexp

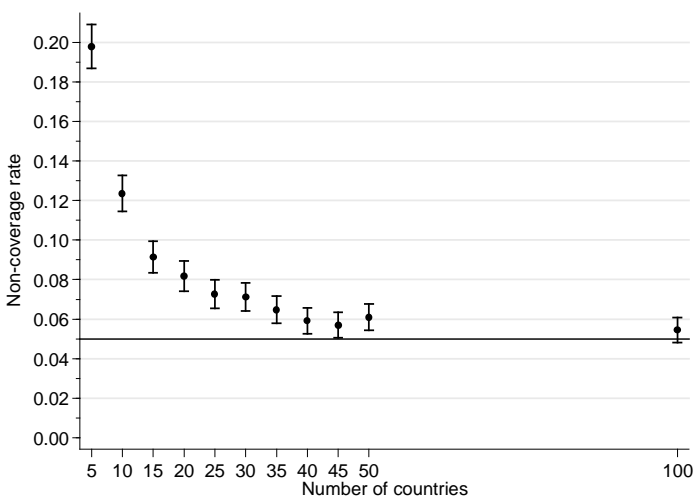

sig_u

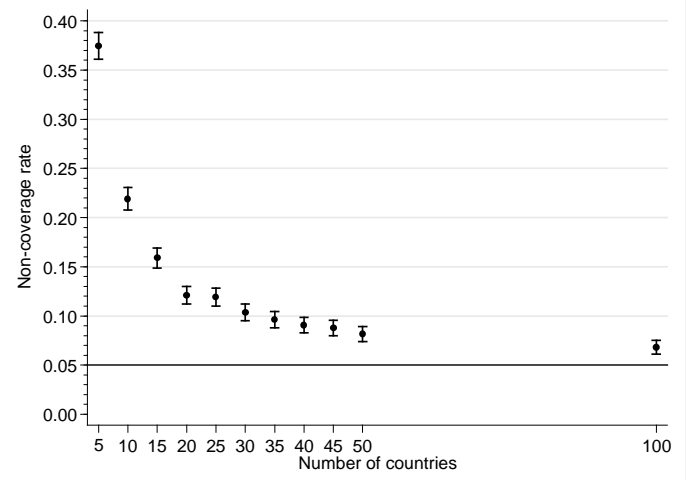


Figure 9. Relative parameter bias (\%): binary logit model with random intercept, two random slopes, country-level regressor and individual-country interaction (Extended model for participation), selected parameters
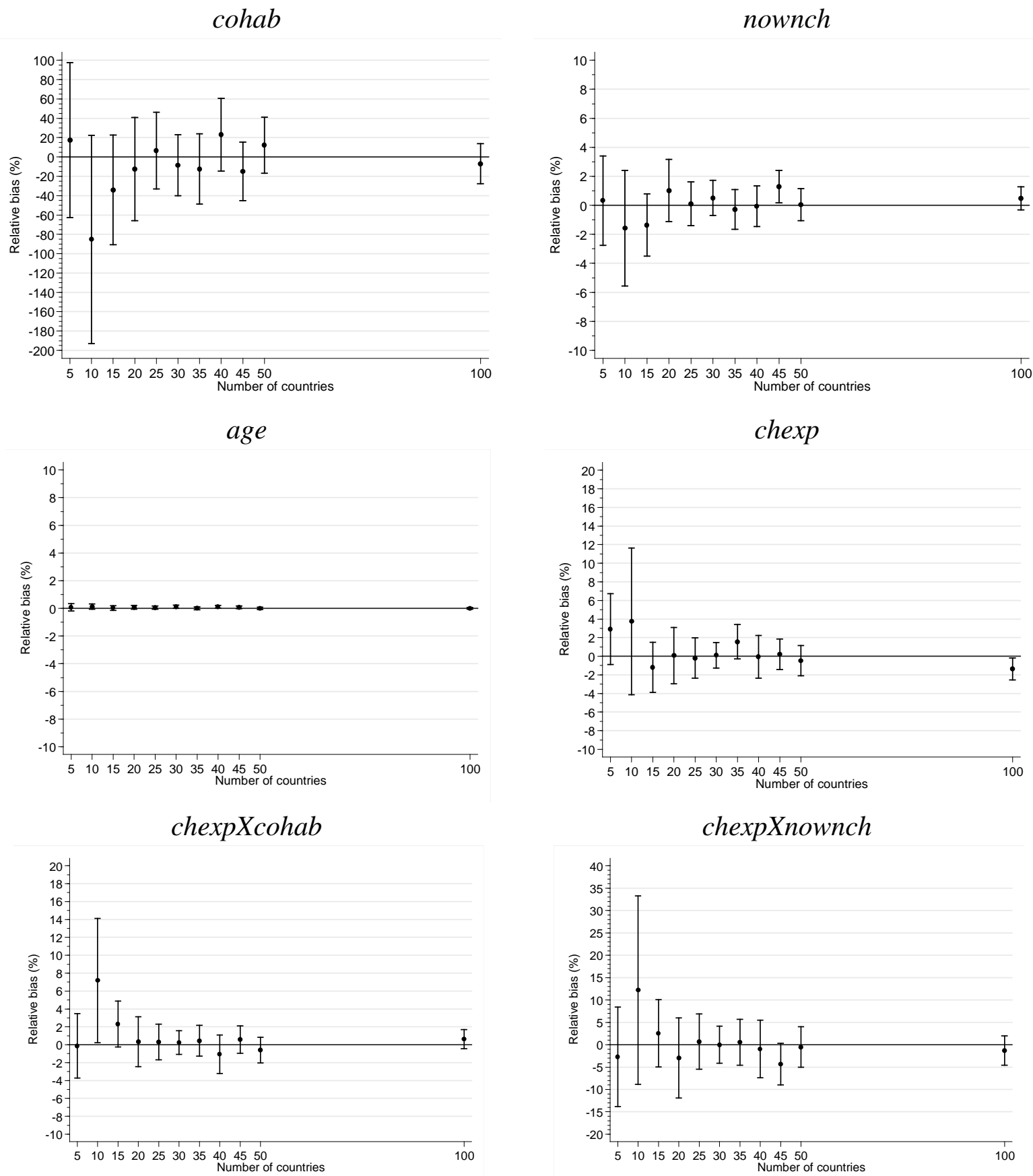

\section{Continued overleaf}


Figure 9 (continued). Relative parameter bias (\%):binary logit model with random intercept, two random slopes, country-level regressor and individual-country interaction (Extended model for participation), selected parameters

sig_b3c

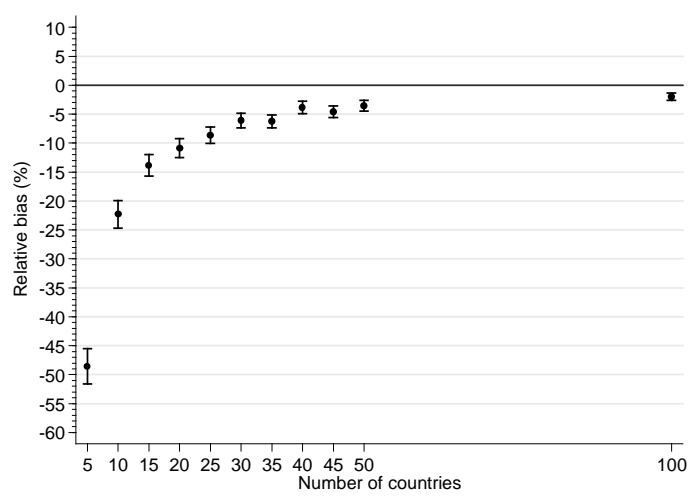

sig_b4c

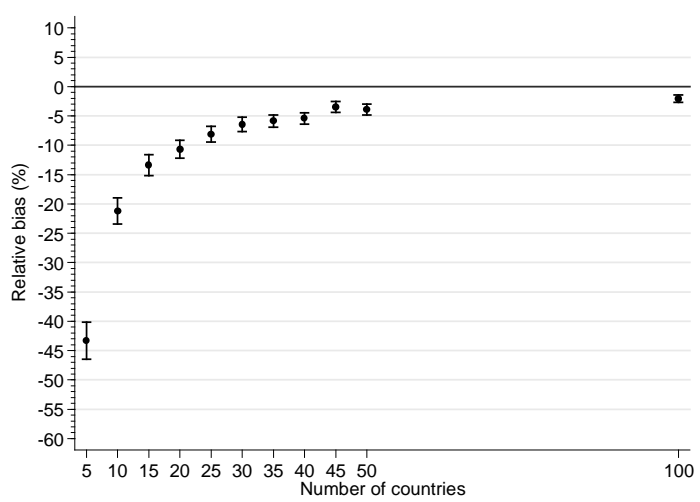

sig_u

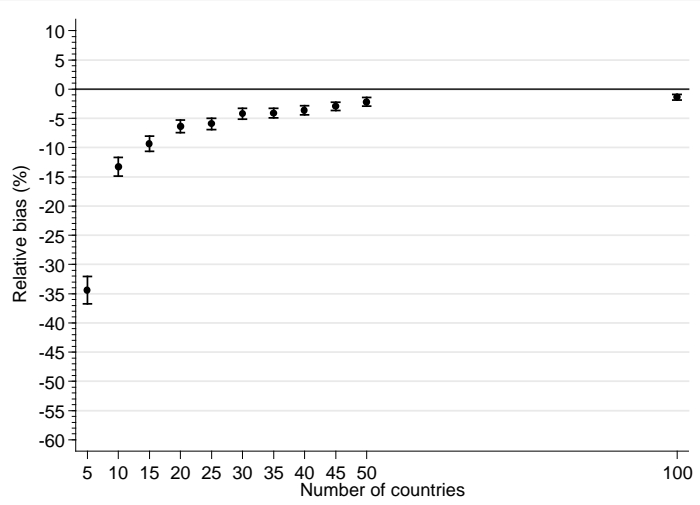


Figure 10. Non-coverage rate: binary logit model with random intercept, two random slopes, country-level regressor and individual-country interaction (Extended model for participation), selected parameters

cohab

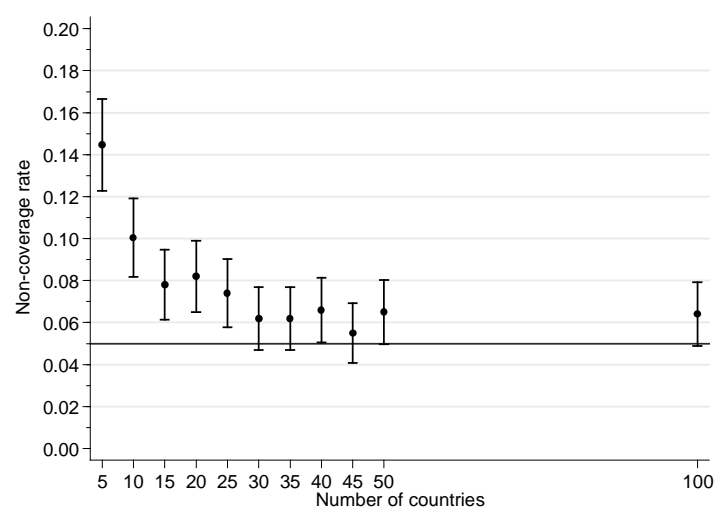

age

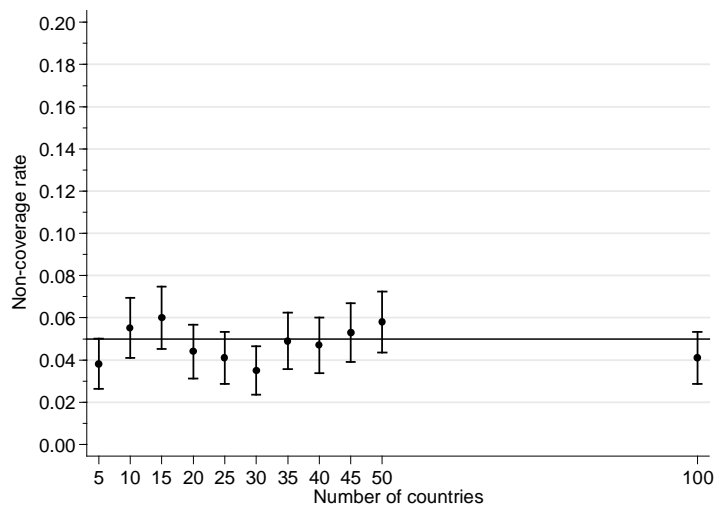

chexpXcohab

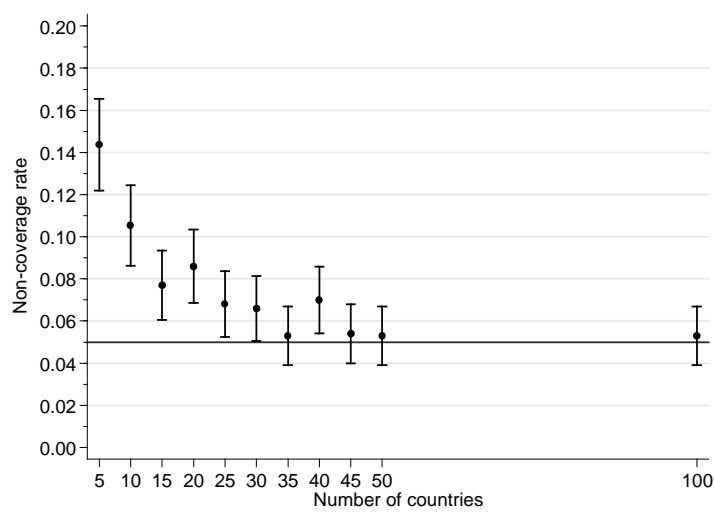

Continued overleaf nownch

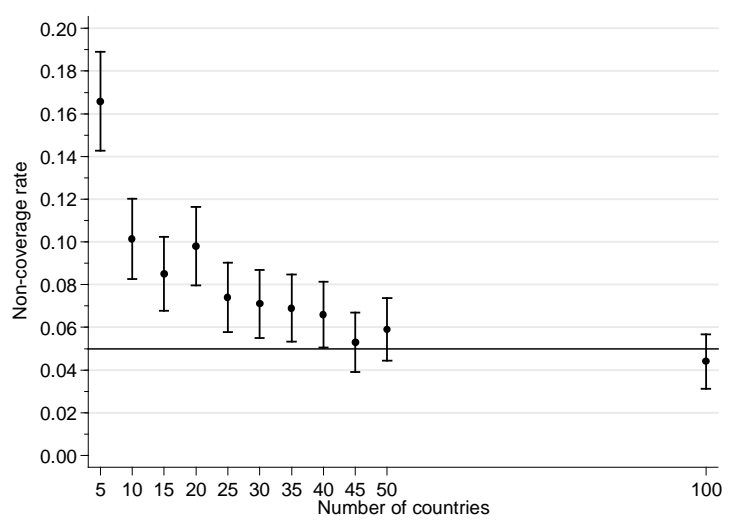

chexp
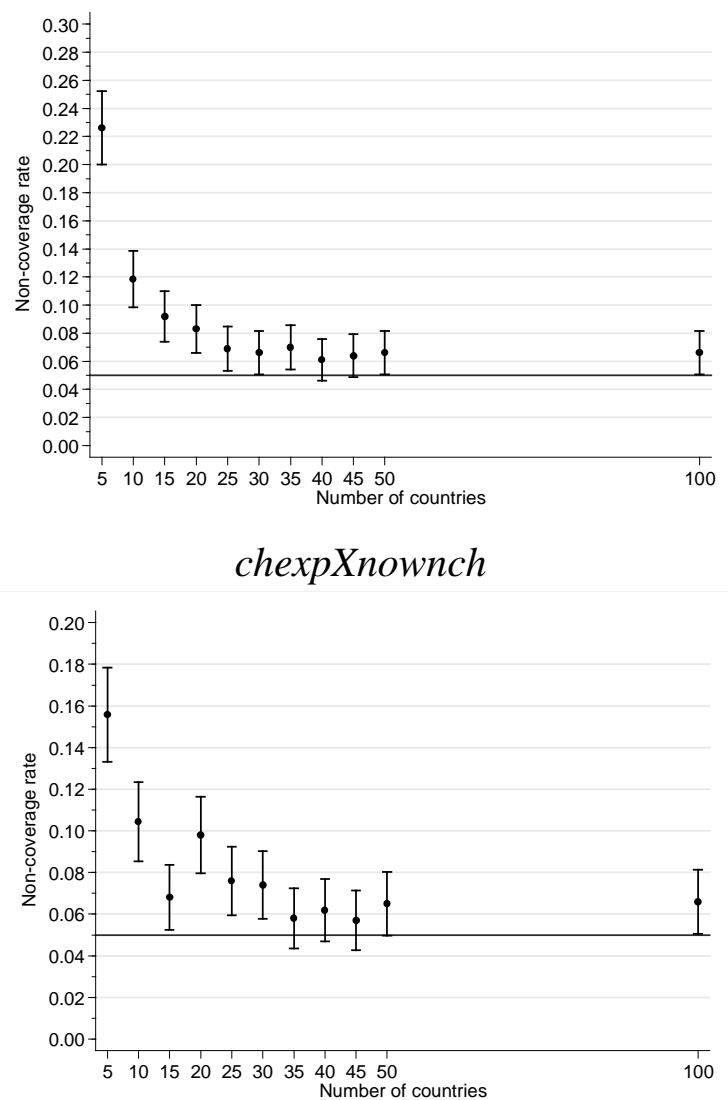
Figure 10 (continued). Non-coverage rate: binary logit model with random intercept, two random slopes, country-level regressor and individual-country interaction (Extended model for participation), selected parameters

sig_b3c

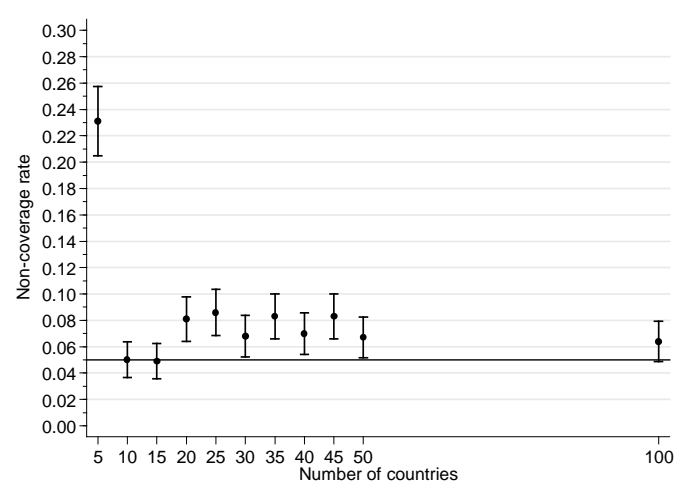

sig_b4c

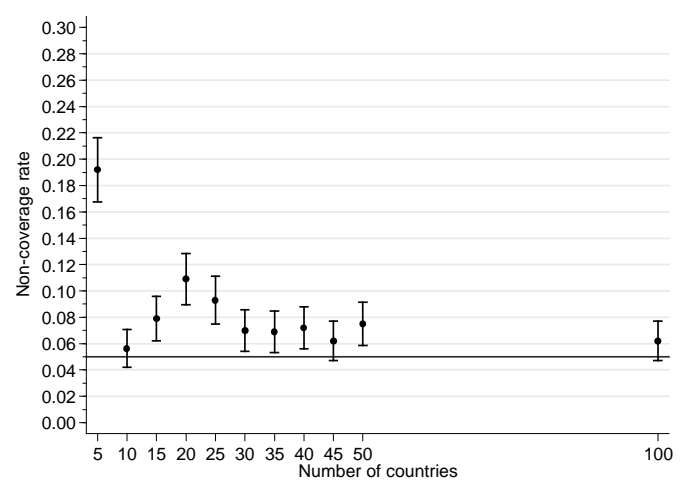

sig_u

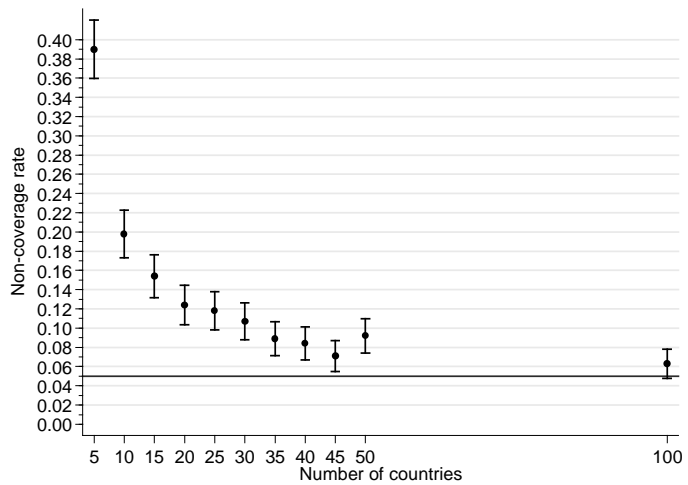


Figure 11. Model of working hours: country-specific intercepts and childcare/pre-primary spending

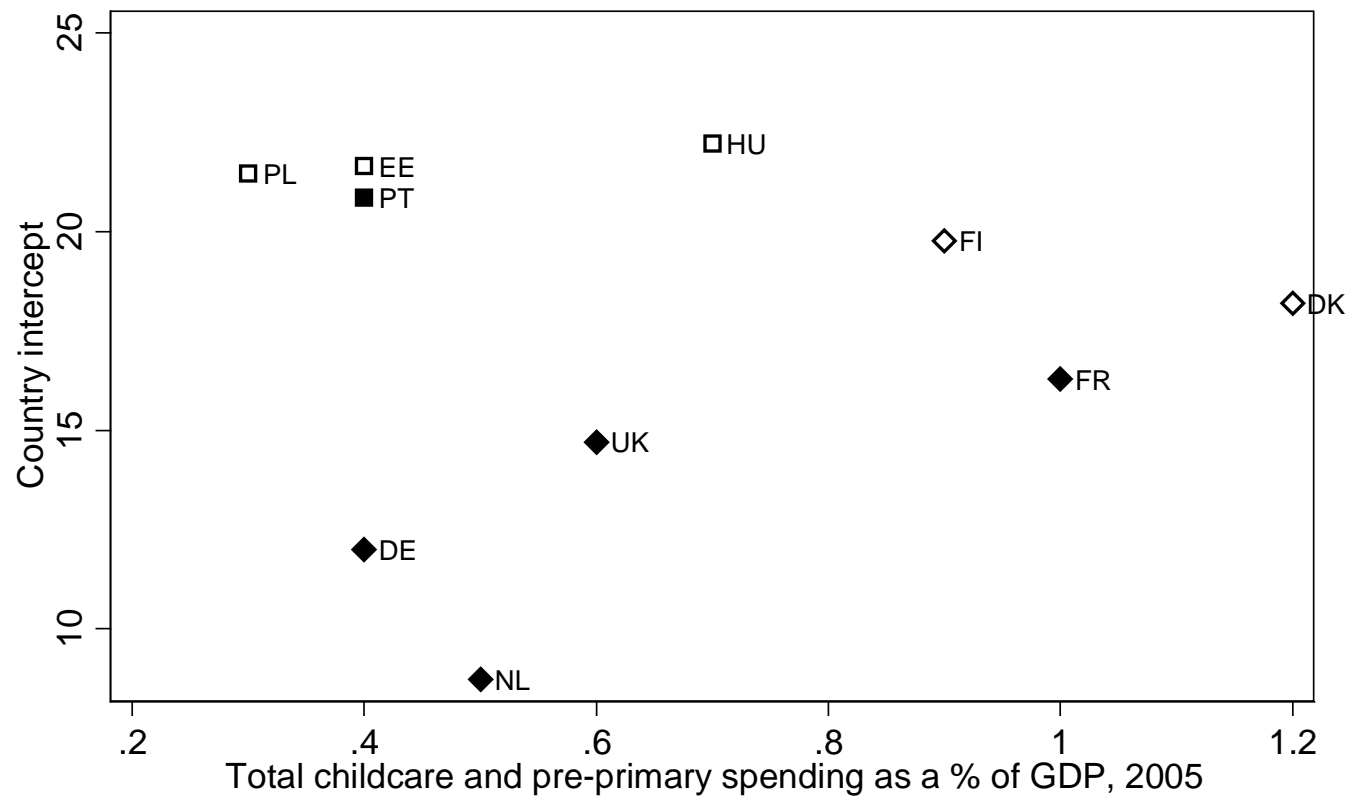
- NW Europe
- S Europe
$\diamond$ Nordic countries
E Europe

Figure 12. Model of work participation: country-specific intercepts and childcare/pre-primary spending (10 countries)

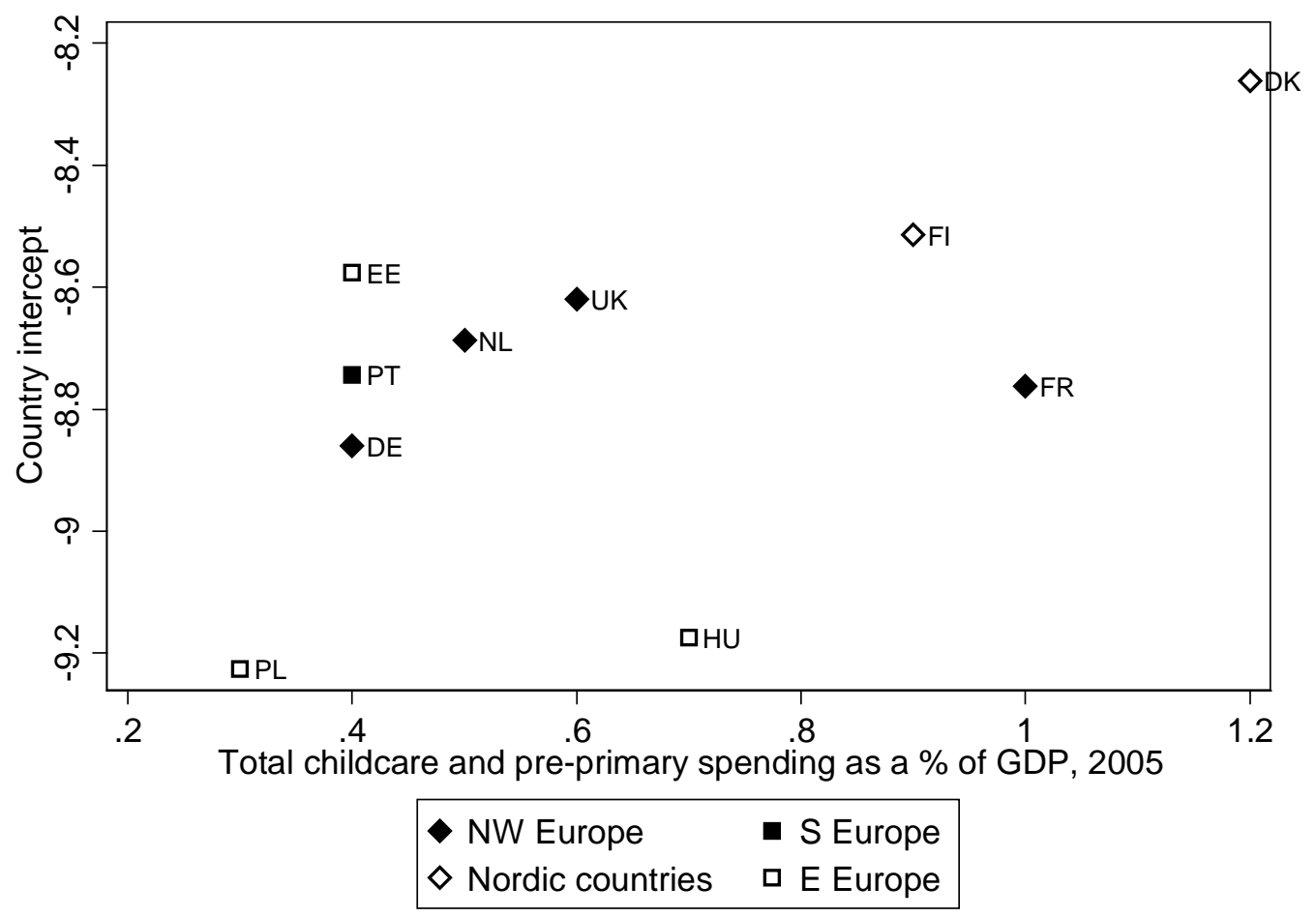




\section{APPENDIX:}

\section{MONTE-CARLO SIMULATION ESTIMATES}

The following tables summarize the Monte Carlo simulations of the models for hours and participation (Basic and Extended).

For each of the four models, we give its specification and summarize the results in two tables: (i) the estimated parameter values and their bias, and (ii) the estimated standard errors and their bias, and the resulting non-coverage rates.

The tables provide the estimates underlying Figures 1-10 in the main body of the paper, as well as additional estimates. 


\section{Appendix: Monte Carlo simulations}

\section{A.1 Hours, Basic Model $(R=10,000)$}

Hours_ic $=22+0.8 *$ age_ic $-0.01 *$ age-squared_ic $-1 *$ cohab_ic $-1.2 *$ nownch_ic $+0.7 *$ isced3_ic $+1.4 *$ isced4_ic $+1.6 *$ isced56_ic $-0.23 *$ chexp_c + u_c + e_ic

u_c $\sim \mathrm{N}\left(0,3.5^{\wedge} 2\right)$, e_ic $\sim \mathrm{N}\left(0,9.5^{\wedge} 2\right), \quad \operatorname{cov}\left(\mathrm{u} \_\mathrm{c}, \mathrm{e} \_\mathrm{ic}\right)=0$ icc $=0.1195122$

Table A1. Hours, Basic Model: estimated parameters

\begin{tabular}{|c|c|c|c|c|c|c|c|}
\hline $\begin{array}{l}\text { Parameter } \\
\text { (true value) }\end{array}$ & $N_{C}$ & Mean & (2) & (3) & $\begin{array}{l}\text { Relative bias, \% } \\
\text { (mean) } \\
(4)\end{array}$ & LB & UB \\
\hline cons & 5 & 22.004 & 21.924 & 22.084 & 0.017 & -0.347 & 0.381 \\
\hline \multirow[t]{10}{*}{ (22) } & 10 & 22.006 & 21.938 & 22.075 & 0.029 & -0.281 & 0.339 \\
\hline & 15 & 21.997 & 21.954 & 22.040 & -0.015 & -0.211 & 0.181 \\
\hline & 20 & 22.075 & 22.022 & 22.128 & 0.341 & 0.102 & 0.581 \\
\hline & 25 & 21.984 & 21.951 & 22.017 & -0.072 & -0.221 & 0.078 \\
\hline & 30 & 22.004 & 21.970 & 22.038 & 0.019 & -0.135 & 0.174 \\
\hline & 35 & 21.994 & 21.968 & 22.020 & -0.027 & -0.145 & 0.091 \\
\hline & 40 & 22.019 & 21.991 & 22.048 & 0.088 & -0.042 & 0.218 \\
\hline & 45 & 22.004 & 21.976 & 22.032 & 0.018 & -0.110 & 0.146 \\
\hline & 50 & 21.994 & 21.970 & 22.018 & -0.028 & -0.137 & 0.081 \\
\hline & 100 & 21.999 & 21.981 & 22.016 & -0.005 & -0.085 & 0.075 \\
\hline age & 5 & 0.801 & 0.799 & 0.803 & 0.149 & -0.100 & 0.398 \\
\hline \multirow[t]{10}{*}{ (0.8) } & 10 & 0.800 & 0.799 & 0.802 & 0.037 & -0.139 & 0.214 \\
\hline & 15 & 0.800 & 0.799 & 0.801 & -0.041 & -0.185 & 0.102 \\
\hline & 20 & 0.799 & 0.798 & 0.801 & -0.064 & -0.191 & 0.063 \\
\hline & 25 & 0.800 & 0.800 & 0.801 & 0.055 & -0.055 & 0.166 \\
\hline & 30 & 0.800 & 0.799 & 0.801 & 0.005 & -0.097 & 0.107 \\
\hline & 35 & 0.801 & 0.800 & 0.801 & 0.077 & -0.018 & 0.171 \\
\hline & 40 & 0.800 & 0.799 & 0.801 & -0.025 & -0.114 & 0.065 \\
\hline & 45 & 0.800 & 0.799 & 0.800 & -0.044 & -0.127 & 0.039 \\
\hline & 50 & 0.800 & 0.800 & 0.801 & 0.033 & -0.047 & 0.112 \\
\hline & 100 & 0.800 & 0.799 & 0.800 & -0.025 & -0.080 & 0.031 \\
\hline cohab & 5 & -1.003 & -1.009 & -0.996 & 0.256 & -0.397 & 0.910 \\
\hline \multirow[t]{10}{*}{$(-1)$} & 10 & -1.000 & -1.005 & -0.995 & -0.001 & -0.455 & 0.453 \\
\hline & 15 & -0.999 & -1.002 & -0.995 & -0.145 & -0.512 & 0.223 \\
\hline & 20 & -1.003 & -1.006 & -1.000 & 0.310 & -0.008 & 0.628 \\
\hline & 25 & -1.002 & -1.005 & -0.999 & 0.226 & -0.057 & 0.510 \\
\hline & 30 & -1.001 & -1.004 & -0.998 & 0.094 & -0.168 & 0.357 \\
\hline & 35 & -1.002 & -1.004 & -1.000 & 0.200 & -0.039 & 0.440 \\
\hline & 40 & -1.001 & -1.003 & -0.999 & 0.119 & -0.109 & 0.347 \\
\hline & 45 & -1.000 & -1.003 & -0.998 & 0.038 & -0.178 & 0.254 \\
\hline & 50 & -1.000 & -1.002 & -0.998 & 0.024 & -0.176 & 0.224 \\
\hline & 100 & -0.999 & -1.000 & -0.997 & -0.146 & -0.290 & -0.003 \\
\hline
\end{tabular}

Continued overleaf 
Table A1 (continued). Hours, Basic Model: estimated parameters

\begin{tabular}{|c|c|c|c|c|c|c|c|}
\hline $\begin{array}{l}\text { Parameter } \\
\text { (true value) }\end{array}$ & $N_{C}$ & Mean & (2) & (3) & $\begin{array}{c}\text { Relative bias, \% } \\
\text { (mean) } \\
(4) \\
\end{array}$ & (5) & (6) \\
\hline Nownch & 5 & -1.202 & -1.205 & -1.199 & 0.161 & -0.085 & 0.407 \\
\hline \multirow[t]{10}{*}{$(-1.2)$} & 10 & -1.199 & -1.201 & -1.197 & -0.103 & -0.275 & 0.069 \\
\hline & 15 & -1.198 & -1.200 & -1.197 & -0.132 & -0.270 & 0.007 \\
\hline & 20 & -1.201 & -1.202 & -1.199 & 0.069 & -0.053 & 0.190 \\
\hline & 25 & -1.199 & -1.201 & -1.198 & -0.046 & -0.154 & 0.062 \\
\hline & 30 & -1.199 & -1.200 & -1.198 & -0.084 & -0.183 & 0.015 \\
\hline & 35 & -1.200 & -1.201 & -1.199 & -0.008 & -0.100 & 0.085 \\
\hline & 40 & -1.200 & -1.201 & -1.199 & -0.021 & -0.106 & 0.063 \\
\hline & 45 & -1.201 & -1.202 & -1.200 & 0.068 & -0.011 & 0.146 \\
\hline & 50 & -1.200 & -1.201 & -1.200 & 0.041 & -0.036 & 0.118 \\
\hline & 100 & -1.200 & -1.201 & -1.199 & 0.011 & -0.043 & 0.066 \\
\hline chexp & 5 & -0.311 & -0.415 & -0.206 & 35.115 & -10.298 & 80.527 \\
\hline \multirow[t]{10}{*}{$(-0.23)$} & 10 & -0.235 & -0.328 & -0.141 & 2.098 & -38.618 & 42.813 \\
\hline & 15 & -0.197 & -0.245 & -0.149 & -14.360 & -35.406 & 6.686 \\
\hline & 20 & -0.364 & -0.460 & -0.269 & 58.421 & 16.968 & 99.874 \\
\hline & 25 & -0.223 & -0.268 & -0.178 & -3.206 & -22.802 & 16.389 \\
\hline & 30 & -0.237 & -0.284 & -0.191 & 3.247 & -17.134 & 23.628 \\
\hline & 35 & -0.237 & -0.268 & -0.207 & 3.191 & -10.005 & 16.387 \\
\hline & 40 & -0.252 & -0.286 & -0.219 & 9.775 & -4.945 & 24.496 \\
\hline & 45 & -0.219 & -0.261 & -0.178 & -4.754 & -22.776 & 13.268 \\
\hline & 50 & -0.228 & -0.262 & -0.194 & -0.748 & -15.440 & 13.945 \\
\hline & 100 & -0.227 & -0.249 & -0.205 & -1.261 & -10.923 & 8.402 \\
\hline sig_u & 5 & 3.221 & 3.194 & 3.248 & -7.967 & -8.736 & -7.199 \\
\hline \multirow[t]{10}{*}{ (3.5) } & 10 & 3.392 & 3.375 & 3.408 & -3.099 & -3.581 & -2.616 \\
\hline & 15 & 3.447 & 3.434 & 3.460 & -1.518 & -1.900 & -1.136 \\
\hline & 20 & 3.459 & 3.448 & 3.471 & -1.165 & -1.494 & -0.836 \\
\hline & 25 & 3.462 & 3.452 & 3.473 & -1.077 & -1.369 & -0.785 \\
\hline & 30 & 3.469 & 3.460 & 3.478 & -0.888 & -1.151 & -0.625 \\
\hline & 35 & 3.481 & 3.472 & 3.489 & -0.555 & -0.797 & -0.312 \\
\hline & 40 & 3.475 & 3.467 & 3.483 & -0.724 & -0.950 & -0.497 \\
\hline & 45 & 3.478 & 3.470 & 3.485 & -0.637 & -0.846 & -0.428 \\
\hline & 50 & 3.481 & 3.474 & 3.488 & -0.533 & -0.731 & -0.334 \\
\hline & 100 & 3.492 & 3.488 & 3.497 & -0.215 & -0.356 & -0.073 \\
\hline sig_e & 5 & 9.500 & 9.498 & 9.502 & 0.000 & -0.019 & 0.020 \\
\hline \multirow[t]{10}{*}{$(9.5)$} & 10 & 9.499 & 9.498 & 9.501 & -0.008 & -0.022 & 0.006 \\
\hline & 15 & 9.499 & 9.498 & 9.500 & -0.008 & -0.020 & 0.003 \\
\hline & 20 & 9.500 & 9.499 & 9.501 & -0.003 & -0.012 & 0.007 \\
\hline & 25 & 9.500 & 9.499 & 9.501 & -0.003 & -0.012 & 0.005 \\
\hline & 30 & 9.500 & 9.499 & 9.501 & -0.001 & -0.009 & 0.007 \\
\hline & 35 & 9.500 & 9.500 & 9.501 & 0.003 & -0.005 & 0.010 \\
\hline & 40 & 9.500 & 9.500 & 9.501 & 0.002 & -0.005 & 0.009 \\
\hline & 45 & 9.500 & 9.499 & 9.501 & -0.000 & -0.007 & 0.006 \\
\hline & 50 & 9.500 & 9.499 & 9.500 & -0.001 & -0.007 & 0.005 \\
\hline & 100 & 9.500 & 9.500 & 9.501 & 0.001 & -0.003 & 0.005 \\
\hline
\end{tabular}

Continued overleaf 
Table A1 (continued). Hours, Basic Model: estimated parameters

\begin{tabular}{lccccccr}
\hline $\begin{array}{l}\text { Parameter } \\
\text { (true value) }\end{array}$ & $N_{C}$ & Mean & LB & UB & $\begin{array}{c}\text { Relative bias, \% } \\
\text { (mean) }\end{array}$ & LB & UB \\
& & $(1)$ & $(2)$ & $(3)$ & $(4)$ & $(5)$ & $(6)$ \\
\hline icc & 5 & 0.112 & 0.111 & 0.114 & -6.207 & -7.481 & -4.933 \\
$(0.120)$ & 10 & 0.116 & 0.115 & 0.117 & -2.530 & -3.358 & -1.702 \\
& 15 & 0.118 & 0.118 & 0.119 & -0.887 & -1.550 & -0.225 \\
& 20 & 0.119 & 0.118 & 0.119 & -0.725 & -1.298 & -0.153 \\
& 25 & 0.118 & 0.118 & 0.119 & -0.850 & -1.359 & -0.340 \\
& 30 & 0.119 & 0.118 & 0.119 & -0.716 & -1.175 & -0.257 \\
& 35 & 0.119 & 0.119 & 0.120 & -0.273 & -0.697 & 0.152 \\
& 40 & 0.119 & 0.118 & 0.119 & -0.655 & -1.052 & -0.258 \\
& 45 & 0.119 & 0.118 & 0.119 & -0.589 & -0.955 & -0.223 \\
& 50 & 0.119 & 0.119 & 0.119 & -0.458 & -0.807 & -0.110 \\
& 100 & 0.119 & 0.119 & 0.120 & -0.139 & -0.388 & 0.110 \\
\hline
\end{tabular}

Notes

(1) mean of distribution of parameter estimates from each Monte-Carlo replication (2), (3): lower and upper bounds of 95\% CI for (1), calculated assuming normality of MC sampling distribution

(4) Relative bias: percentage difference between (1) and 'true' parameter value (5), (6): lower and upper bounds of 95\% CI for (4), calculated assuming normality of MC sampling distribution 
Table A2. Hours, Basic Model: estimated standard errors and non-coverage rates

\begin{tabular}{|c|c|c|c|c|c|c|c|c|c|}
\hline Parameter & $N_{C}$ & $\begin{array}{c}\text { Empirical } \\
\text { SE } \\
(1) \\
\end{array}$ & $\begin{array}{c}\text { Analytic } \\
\text { al SE } \\
(2) \\
\end{array}$ & LB & UB & $\begin{array}{c}\text { Relative } \\
\text { difference, } \\
\% \\
(5) \\
\end{array}$ & $\begin{array}{l}\text { Non- } \\
\text { coverage } \\
\text { rate, \% } \\
(6) \\
\end{array}$ & LB & UB \\
\hline \multirow[t]{11}{*}{ cons } & 5 & 4.088 & 3.884 & 3.861 & 3.907 & -4.981 & 0.097 & 0.092 & 0.103 \\
\hline & 10 & 3.479 & 3.440 & 3.426 & 3.454 & -1.128 & 0.070 & 0.065 & 0.075 \\
\hline & 15 & 2.199 & 2.182 & 2.176 & 2.188 & -0.745 & 0.062 & 0.057 & 0.067 \\
\hline & 20 & 2.688 & 2.675 & 2.667 & 2.683 & -0.472 & 0.060 & 0.055 & 0.065 \\
\hline & 25 & 1.678 & 1.670 & 1.667 & 1.674 & -0.483 & 0.056 & 0.051 & 0.060 \\
\hline & 30 & 1.734 & 1.712 & 1.709 & 1.716 & -1.278 & 0.058 & 0.053 & 0.062 \\
\hline & 35 & 1.325 & 1.319 & 1.317 & 1.321 & -0.457 & 0.053 & 0.049 & 0.057 \\
\hline & 40 & 1.454 & 1.462 & 1.460 & 1.465 & 0.577 & 0.052 & 0.048 & 0.057 \\
\hline & 45 & 1.437 & 1.432 & 1.430 & 1.435 & -0.283 & 0.052 & 0.048 & 0.057 \\
\hline & 50 & 1.221 & 1.208 & 1.206 & 1.210 & -1.061 & 0.056 & 0.051 & 0.060 \\
\hline & 100 & 0.894 & 0.886 & 0.885 & 0.887 & -0.914 & 0.051 & 0.047 & 0.056 \\
\hline \multirow[t]{11}{*}{ age } & 5 & 0.101 & 0.101 & 0.101 & 0.101 & -0.396 & 0.051 & 0.047 & 0.055 \\
\hline & 10 & 0.072 & 0.072 & 0.072 & 0.072 & -0.525 & 0.051 & 0.046 & 0.055 \\
\hline & 15 & 0.059 & 0.059 & 0.059 & 0.059 & -0.045 & 0.050 & 0.045 & 0.054 \\
\hline & 20 & 0.052 & 0.051 & 0.051 & 0.051 & -1.706 & 0.055 & 0.050 & 0.059 \\
\hline & 25 & 0.045 & 0.046 & 0.046 & 0.046 & 0.926 & 0.047 & 0.043 & 0.051 \\
\hline & 30 & 0.042 & 0.042 & 0.042 & 0.042 & 0.079 & 0.052 & 0.048 & 0.057 \\
\hline & 35 & 0.039 & 0.039 & 0.039 & 0.039 & 0.161 & 0.052 & 0.048 & 0.056 \\
\hline & 40 & 0.037 & 0.036 & 0.036 & 0.036 & -0.765 & 0.049 & 0.045 & 0.054 \\
\hline & 45 & 0.034 & 0.034 & 0.034 & 0.034 & 0.471 & 0.048 & 0.043 & 0.052 \\
\hline & 50 & 0.032 & 0.032 & 0.032 & 0.032 & -0.351 & 0.048 & 0.044 & 0.053 \\
\hline & 100 & 0.023 & 0.023 & 0.023 & 0.023 & 0.690 & 0.049 & 0.045 & 0.054 \\
\hline \multirow[t]{11}{*}{ cohab } & 5 & 0.333 & 0.330 & 0.330 & 0.330 & -1.083 & 0.052 & 0.047 & 0.056 \\
\hline & 10 & 0.232 & 0.229 & 0.229 & 0.229 & -1.058 & 0.052 & 0.047 & 0.056 \\
\hline & 15 & 0.188 & 0.186 & 0.186 & 0.186 & -0.600 & 0.050 & 0.046 & 0.054 \\
\hline & 20 & 0.162 & 0.163 & 0.163 & 0.163 & 0.501 & 0.049 & 0.045 & 0.053 \\
\hline & 25 & 0.145 & 0.146 & 0.146 & 0.146 & 0.759 & 0.049 & 0.044 & 0.053 \\
\hline & 30 & 0.134 & 0.134 & 0.134 & 0.134 & -0.257 & 0.051 & 0.047 & 0.055 \\
\hline & 35 & 0.122 & 0.124 & 0.124 & 0.124 & 1.053 & 0.048 & 0.044 & 0.052 \\
\hline & 40 & 0.116 & 0.116 & 0.116 & 0.116 & -0.405 & 0.050 & 0.046 & 0.054 \\
\hline & 45 & 0.110 & 0.109 & 0.109 & 0.109 & -1.066 & 0.049 & 0.045 & 0.054 \\
\hline & 50 & 0.102 & 0.103 & 0.103 & 0.103 & 1.426 & 0.046 & 0.042 & 0.050 \\
\hline & 100 & 0.073 & 0.073 & 0.073 & 0.073 & -0.448 & 0.051 & 0.046 & 0.055 \\
\hline \multirow[t]{11}{*}{ nownch } & 5 & 0.151 & 0.150 & 0.150 & 0.150 & -0.344 & 0.052 & 0.047 & 0.056 \\
\hline & 10 & 0.105 & 0.104 & 0.104 & 0.104 & -0.890 & 0.055 & 0.050 & 0.059 \\
\hline & 15 & 0.085 & 0.085 & 0.085 & 0.085 & 0.586 & 0.048 & 0.044 & 0.052 \\
\hline & 20 & 0.074 & 0.074 & 0.074 & 0.074 & -0.430 & 0.051 & 0.047 & 0.055 \\
\hline & 25 & 0.066 & 0.066 & 0.066 & 0.066 & 0.279 & 0.047 & 0.043 & 0.051 \\
\hline & 30 & 0.061 & 0.060 & 0.060 & 0.060 & -0.721 & 0.052 & 0.047 & 0.056 \\
\hline & 35 & 0.056 & 0.056 & 0.056 & 0.056 & -0.835 & 0.051 & 0.047 & 0.055 \\
\hline & 40 & 0.052 & 0.052 & 0.052 & 0.052 & 0.729 & 0.050 & 0.046 & 0.054 \\
\hline & 45 & 0.048 & 0.049 & 0.049 & 0.049 & 1.858 & 0.044 & 0.040 & 0.048 \\
\hline & 50 & 0.047 & 0.047 & 0.047 & 0.047 & -0.146 & 0.051 & 0.047 & 0.056 \\
\hline & 100 & 0.033 & 0.033 & 0.033 & 0.033 & -0.637 & 0.050 & 0.046 & 0.054 \\
\hline
\end{tabular}

Continued overleaf 
Table A2 (contd.). Hours, Basic Model: estimated standard errors and non-coverage rates

\begin{tabular}{|c|c|c|c|c|c|c|c|c|c|}
\hline Parameter & $N_{C}$ & $\begin{array}{c}\text { Empirical } \\
\text { SE } \\
(1)\end{array}$ & $\begin{array}{c}\text { Analytic } \\
\text { al SE }\end{array}$ & LB & UB & $\begin{array}{c}\text { Relative } \\
\text { difference, } \\
\% \\
(5) \\
\end{array}$ & $\begin{array}{l}\text { Non- } \\
\text { coverage } \\
\text { rate, \% } \\
(6) \\
\end{array}$ & LB & UB \\
\hline \multirow[t]{11}{*}{ chexp } & 5 & 5.329 & 4.857 & 4.817 & 4.897 & -8.857 & 0.149 & 0.142 & 0.156 \\
\hline & 10 & 4.778 & 4.673 & 4.650 & 4.696 & -2.195 & 0.080 & 0.075 & 0.085 \\
\hline & 15 & 2.470 & 2.425 & 2.416 & 2.435 & -1.803 & 0.070 & 0.065 & 0.075 \\
\hline & 20 & 4.864 & 4.847 & 4.831 & 4.863 & -0.352 & 0.063 & 0.058 & 0.067 \\
\hline & 25 & 2.300 & 2.283 & 2.277 & 2.290 & -0.708 & 0.063 & 0.059 & 0.068 \\
\hline & 30 & 2.392 & 2.346 & 2.339 & 2.352 & -1.923 & 0.063 & 0.058 & 0.068 \\
\hline & 35 & 1.549 & 1.542 & 1.538 & 1.546 & -0.418 & 0.058 & 0.054 & 0.063 \\
\hline & 40 & 1.727 & 1.724 & 1.720 & 1.728 & -0.221 & 0.062 & 0.057 & 0.066 \\
\hline & 45 & 2.115 & 2.091 & 2.087 & 2.096 & -1.113 & 0.059 & 0.054 & 0.064 \\
\hline & 50 & 1.724 & 1.707 & 1.704 & 1.711 & -0.982 & 0.055 & 0.051 & 0.060 \\
\hline & 100 & 1.134 & 1.121 & 1.120 & 1.123 & -1.094 & 0.055 & 0.051 & 0.059 \\
\hline \multirow[t]{11}{*}{ sig_u } & 5 & 1.373 & 1.330 & 1.319 & 1.341 & -3.129 & 0.177 & 0.169 & 0.184 \\
\hline & 10 & 0.862 & 0.855 & 0.851 & 0.859 & -0.782 & 0.108 & 0.102 & 0.114 \\
\hline & 15 & 0.682 & 0.681 & 0.679 & 0.684 & -0.026 & 0.084 & 0.079 & 0.090 \\
\hline & 20 & 0.587 & 0.581 & 0.579 & 0.583 & -1.083 & 0.078 & 0.073 & 0.083 \\
\hline & 25 & 0.522 & 0.514 & 0.513 & 0.516 & -1.373 & 0.071 & 0.066 & 0.076 \\
\hline & 30 & 0.470 & 0.467 & 0.466 & 0.468 & -0.650 & 0.068 & 0.063 & 0.073 \\
\hline & 35 & 0.433 & 0.432 & 0.431 & 0.433 & -0.296 & 0.064 & 0.059 & 0.068 \\
\hline & 40 & 0.405 & 0.402 & 0.401 & 0.402 & -0.725 & 0.064 & 0.059 & 0.068 \\
\hline & 45 & 0.373 & 0.378 & 0.377 & 0.379 & 1.273 & 0.059 & 0.055 & 0.064 \\
\hline & 50 & 0.355 & 0.358 & 0.357 & 0.359 & 0.899 & 0.058 & 0.053 & 0.063 \\
\hline & 100 & 0.253 & 0.251 & 0.251 & 0.252 & -0.562 & 0.056 & 0.051 & 0.060 \\
\hline \multirow[t]{11}{*}{ sig_e } & 5 & 0.094 & 0.095 & 0.095 & 0.095 & 0.764 & 0.045 & 0.041 & 0.049 \\
\hline & 10 & 0.067 & 0.067 & 0.067 & 0.067 & 0.113 & 0.047 & 0.043 & 0.052 \\
\hline & 15 & 0.055 & 0.055 & 0.055 & 0.055 & -0.095 & 0.052 & 0.048 & 0.056 \\
\hline & 20 & 0.047 & 0.048 & 0.048 & 0.048 & 0.904 & 0.047 & 0.042 & 0.051 \\
\hline & 25 & 0.043 & 0.043 & 0.043 & 0.043 & -0.290 & 0.052 & 0.048 & 0.056 \\
\hline & 30 & 0.039 & 0.039 & 0.039 & 0.039 & 0.513 & 0.051 & 0.046 & 0.055 \\
\hline & 35 & 0.036 & 0.036 & 0.036 & 0.036 & -0.690 & 0.053 & 0.049 & 0.058 \\
\hline & 40 & 0.033 & 0.034 & 0.034 & 0.034 & 0.323 & 0.050 & 0.045 & 0.054 \\
\hline & 45 & 0.032 & 0.032 & 0.032 & 0.032 & 0.489 & 0.051 & 0.047 & 0.056 \\
\hline & 50 & 0.030 & 0.030 & 0.030 & 0.030 & 0.145 & 0.050 & 0.046 & 0.054 \\
\hline & 100 & 0.021 & 0.021 & 0.021 & 0.021 & 0.020 & 0.051 & 0.047 & 0.056 \\
\hline
\end{tabular}

Notes

(1): Empirical SE: standard deviation of distribution of parameter estimates from each Monte-Carlo replication

(2): Analytical SE: mean of distribution of SE estimates from each Monte-Carlo replication

(3), (4): lower and upper bounds of 95\% CI for (1), calculated assuming normality of MC sampling distribution

(5): Relative difference: percentage difference between (2) and (1)

(6): Non-coverage rate: proportion of MC replications for which estimated 95\% CI did not contain the true parameter (CI calculated using fitted SEs).

(7), (8): lower and upper bounds of 95\% CI for (6), calculated assuming normality of MC sampling distribution 


\section{A.2 Hours, Extended Model $(R=5,000)$}

Hours_ic $=22+0.8 *$ age_ic $-0.01 *$ age-squared_ic

$-(1+\mathrm{b} 3 \mathrm{c}) *$ cohab_ic $-(1.2+\mathrm{b} 4 \mathrm{c}) *$ nownch_ic

$+0.7 *$ isced3_ic $+1.4 *$ isced4_ic $+1.6 *$ isced56_ic

$-2.7 *$ chexp_c $+2.4 *$ (chexp_c X cohab_ic) $+0.7 *$ (chexp_c X nownch_ic)

+ u_c + e_ic

u_c $\sim \mathrm{N}(0,2.4 \wedge 2)$, e_ic $\sim \mathrm{N}\left(0,9.4^{\wedge} 2\right), \quad$ cov $($ u_c, e_ic $)=0$ icc $=0.06119847$

sig_b3c $=1.2$, sig_b4c $=1.2$

Table A3. Hours, Extended Model: estimated parameters

\begin{tabular}{|c|c|c|c|c|c|c|c|}
\hline $\begin{array}{l}\text { Parameter } \\
\text { (true value) }\end{array}$ & $N_{C}$ & (1) & $(2)$ & (3) & $\begin{array}{c}\text { Relative bias, \% } \\
\text { (mean) } \\
(4)\end{array}$ & (5) & (6) \\
\hline cons & 5 & 22.045 & 21.958 & 22.132 & 0.205 & -0.190 & 0.601 \\
\hline \multirow[t]{10}{*}{ (22) } & 10 & 22.022 & 21.948 & 22.096 & 0.099 & -0.236 & 0.435 \\
\hline & 15 & 22.024 & 21.975 & 22.072 & 0.107 & -0.112 & 0.326 \\
\hline & 20 & 21.995 & 21.940 & 22.051 & -0.020 & -0.272 & 0.231 \\
\hline & 25 & 22.002 & 21.965 & 22.039 & 0.010 & -0.157 & 0.177 \\
\hline & 30 & 21.991 & 21.954 & 22.029 & -0.039 & -0.208 & 0.131 \\
\hline & 35 & 21.994 & 21.964 & 22.023 & -0.029 & -0.163 & 0.104 \\
\hline & 40 & 21.993 & 21.962 & 22.025 & -0.030 & -0.172 & 0.112 \\
\hline & 45 & 22.018 & 21.987 & 22.049 & 0.082 & -0.058 & 0.222 \\
\hline & 50 & 21.977 & 21.950 & 22.004 & -0.103 & -0.225 & 0.019 \\
\hline & 100 & 22.012 & 21.993 & 22.031 & 0.055 & -0.033 & 0.142 \\
\hline age & 5 & 0.799 & 0.797 & 0.802 & -0.069 & -0.426 & 0.287 \\
\hline \multirow[t]{10}{*}{$(0.8)$} & 10 & 0.799 & 0.797 & 0.801 & -0.083 & -0.329 & 0.163 \\
\hline & 15 & 0.799 & 0.798 & 0.801 & -0.076 & -0.278 & 0.126 \\
\hline & 20 & 0.800 & 0.798 & 0.801 & -0.034 & -0.209 & 0.141 \\
\hline & 25 & 0.800 & 0.798 & 0.801 & -0.035 & -0.192 & 0.123 \\
\hline & 30 & 0.799 & 0.798 & 0.800 & -0.087 & -0.229 & 0.055 \\
\hline & 35 & 0.800 & 0.798 & 0.801 & -0.055 & -0.188 & 0.078 \\
\hline & 40 & 0.800 & 0.799 & 0.801 & 0.001 & -0.121 & 0.123 \\
\hline & 45 & 0.800 & 0.799 & 0.801 & -0.035 & -0.152 & 0.083 \\
\hline & 50 & 0.801 & 0.800 & 0.802 & 0.086 & -0.026 & 0.198 \\
\hline & 100 & 0.799 & 0.799 & 0.800 & -0.068 & -0.147 & 0.011 \\
\hline cohab & 5 & -1.024 & -1.063 & -0.985 & 2.390 & -1.470 & 6.250 \\
\hline \multirow[t]{10}{*}{$(-1)$} & 10 & -0.984 & -1.019 & -0.948 & -1.629 & -5.162 & 1.904 \\
\hline & 15 & -1.006 & -1.027 & -0.986 & 0.635 & -1.417 & 2.687 \\
\hline & 20 & -1.001 & -1.028 & -0.974 & 0.094 & -2.608 & 2.796 \\
\hline & 25 & -0.995 & -1.011 & -0.980 & -0.467 & -2.046 & 1.111 \\
\hline & 30 & -1.010 & -1.027 & -0.994 & 1.033 & -0.615 & 2.680 \\
\hline & 35 & -1.005 & -1.017 & -0.994 & 0.547 & -0.631 & 1.725 \\
\hline & 40 & -0.999 & -1.013 & -0.984 & -0.136 & -1.551 & 1.278 \\
\hline & 45 & -1.003 & -1.017 & -0.989 & 0.326 & -1.070 & 1.722 \\
\hline & 50 & -1.004 & -1.016 & -0.993 & 0.436 & -0.694 & 1.565 \\
\hline & 100 & -1.003 & -1.012 & -0.995 & 0.314 & -0.538 & 1.167 \\
\hline
\end{tabular}

Continued overleaf 
Table A3 (continued). Hours, Extended Model: estimated parameters

\begin{tabular}{|c|c|c|c|c|c|c|c|}
\hline $\begin{array}{l}\text { Parameter } \\
\text { (true value) }\end{array}$ & $N_{C}$ & $(1)$ & $(2)$ & (3) & $\begin{array}{l}\text { Relative bias, \% } \\
\text { (mean) } \\
(4)\end{array}$ & (5) & (6) \\
\hline nownch & 5 & -1.201 & -1.236 & -1.166 & 0.051 & -2.866 & 2.967 \\
\hline \multirow[t]{10}{*}{$(-1.2)$} & 10 & -1.182 & -1.213 & -1.150 & -1.516 & -4.129 & 1.096 \\
\hline & 15 & -1.190 & -1.209 & -1.172 & -0.802 & -2.334 & 0.730 \\
\hline & 20 & -1.198 & -1.222 & -1.173 & -0.191 & -2.240 & 1.858 \\
\hline & 25 & -1.197 & -1.211 & -1.183 & -0.254 & -1.412 & 0.904 \\
\hline & 30 & -1.194 & -1.209 & -1.179 & -0.494 & -1.756 & 0.769 \\
\hline & 35 & -1.192 & -1.202 & -1.181 & -0.674 & -1.552 & 0.204 \\
\hline & 40 & -1.203 & -1.216 & -1.191 & 0.271 & -0.761 & 1.303 \\
\hline & 45 & -1.194 & -1.206 & -1.182 & -0.491 & -1.520 & 0.539 \\
\hline & 50 & -1.197 & -1.207 & -1.187 & -0.263 & -1.114 & 0.588 \\
\hline & 100 & -1.194 & -1.202 & -1.187 & -0.484 & -1.113 & 0.146 \\
\hline chexp & 5 & -2.734 & -2.836 & -2.631 & 1.244 & -2.566 & 5.053 \\
\hline \multirow[t]{10}{*}{$(-2.7)$} & 10 & -2.719 & -2.813 & -2.626 & 0.717 & -2.750 & 4.183 \\
\hline & 15 & -2.716 & -2.764 & -2.667 & 0.589 & -1.205 & 2.383 \\
\hline & 20 & -2.681 & -2.776 & -2.586 & -0.691 & -4.211 & 2.829 \\
\hline & 25 & -2.693 & -2.739 & -2.647 & -0.269 & -1.981 & 1.444 \\
\hline & 30 & -2.655 & -2.702 & -2.608 & -1.651 & -3.392 & 0.090 \\
\hline & 35 & -2.687 & -2.717 & -2.658 & -0.463 & -1.572 & 0.645 \\
\hline & 40 & -2.688 & -2.722 & -2.654 & -0.437 & -1.688 & 0.814 \\
\hline & 45 & -2.722 & -2.763 & -2.682 & 0.825 & -0.682 & 2.332 \\
\hline & 50 & -2.691 & -2.724 & -2.658 & -0.338 & -1.568 & 0.892 \\
\hline & 100 & -2.706 & -2.728 & -2.684 & 0.226 & -0.575 & 1.028 \\
\hline chexpXcohab & 5 & 2.412 & 2.355 & 2.468 & 0.495 & -1.863 & 2.853 \\
\hline \multirow[t]{10}{*}{$(2.4)$} & 10 & 2.397 & 2.344 & 2.450 & -0.132 & -2.344 & 2.079 \\
\hline & 15 & 2.409 & 2.382 & 2.437 & 0.391 & -0.745 & 1.526 \\
\hline & 20 & 2.416 & 2.363 & 2.470 & 0.677 & -1.547 & 2.900 \\
\hline & 25 & 2.387 & 2.361 & 2.413 & -0.552 & -1.626 & 0.522 \\
\hline & 30 & 2.410 & 2.384 & 2.436 & 0.420 & -0.666 & 1.505 \\
\hline & 35 & 2.412 & 2.395 & 2.429 & 0.504 & -0.202 & 1.210 \\
\hline & 40 & 2.402 & 2.383 & 2.421 & 0.100 & -0.693 & 0.893 \\
\hline & 45 & 2.411 & 2.388 & 2.434 & 0.468 & -0.489 & 1.426 \\
\hline & 50 & 2.413 & 2.394 & 2.431 & 0.532 & -0.243 & 1.307 \\
\hline & 100 & 2.409 & 2.397 & 2.422 & 0.380 & -0.140 & 0.900 \\
\hline chexpXnownch & 5 & 0.703 & 0.650 & 0.755 & 0.362 & -7.129 & 7.852 \\
\hline \multirow[t]{10}{*}{$(0.7)$} & 10 & 0.685 & 0.638 & 0.732 & -2.138 & -8.845 & 4.568 \\
\hline & 15 & 0.688 & 0.664 & 0.713 & -1.691 & -5.170 & 1.788 \\
\hline & 20 & 0.710 & 0.661 & 0.758 & 1.379 & -5.508 & 8.267 \\
\hline & 25 & 0.695 & 0.672 & 0.717 & -0.746 & -3.989 & 2.498 \\
\hline & 30 & 0.695 & 0.672 & 0.719 & -0.656 & -4.054 & 2.742 \\
\hline & 35 & 0.691 & 0.676 & 0.706 & -1.241 & -3.386 & 0.905 \\
\hline & 40 & 0.705 & 0.688 & 0.721 & 0.645 & -1.752 & 3.042 \\
\hline & 45 & 0.690 & 0.669 & 0.710 & -1.450 & -4.375 & 1.475 \\
\hline & 50 & 0.697 & 0.680 & 0.714 & -0.449 & -2.866 & 1.968 \\
\hline & 100 & 0.695 & 0.684 & 0.706 & -0.714 & -2.303 & 0.875 \\
\hline
\end{tabular}

Continued overleaf 
Table A3 (continued). Hours, Extended Model: estimated parameters

\begin{tabular}{|c|c|c|c|c|c|c|c|}
\hline $\begin{array}{l}\text { Parameter } \\
\text { (true value) }\end{array}$ & $N_{C}$ & (1) & $(2)$ & (3) & $\begin{array}{l}\text { Relative bias, \% } \\
\text { (mean) } \\
(4) \\
\end{array}$ & (5) & (6) \\
\hline sig_u & 5 & 2.199 & 2.170 & 2.227 & -8.392 & -9.583 & -7.202 \\
\hline \multirow[t]{10}{*}{$(2.4)$} & 10 & 2.318 & 2.301 & 2.336 & -3.404 & -4.125 & -2.682 \\
\hline & 15 & 2.356 & 2.342 & 2.370 & -1.828 & -2.396 & -1.260 \\
\hline & 20 & 2.366 & 2.355 & 2.378 & -1.403 & -1.884 & -0.922 \\
\hline & 25 & 2.373 & 2.363 & 2.383 & -1.124 & -1.551 & -0.697 \\
\hline & 30 & 2.375 & 2.366 & 2.385 & -1.037 & -1.431 & -0.643 \\
\hline & 35 & 2.384 & 2.375 & 2.392 & -0.678 & -1.032 & -0.324 \\
\hline & 40 & 2.388 & 2.380 & 2.396 & -0.515 & -0.850 & -0.180 \\
\hline & 45 & 2.380 & 2.373 & 2.388 & -0.815 & -1.130 & -0.500 \\
\hline & 50 & 2.390 & 2.383 & 2.398 & -0.399 & -0.697 & -0.101 \\
\hline & 100 & 2.391 & 2.386 & 2.396 & -0.376 & -0.582 & -0.171 \\
\hline sig_e & 5 & 9.401 & 9.398 & 9.404 & 0.010 & -0.018 & 0.038 \\
\hline \multirow[t]{10}{*}{$(9.4)$} & 10 & 9.400 & 9.398 & 9.402 & 0.001 & -0.019 & 0.020 \\
\hline & 15 & 9.399 & 9.398 & 9.401 & -0.005 & -0.022 & 0.011 \\
\hline & 20 & 9.400 & 9.399 & 9.401 & -0.000 & -0.014 & 0.014 \\
\hline & 25 & 9.400 & 9.399 & 9.401 & 0.001 & -0.012 & 0.013 \\
\hline & 30 & 9.400 & 9.399 & 9.401 & 0.001 & -0.011 & 0.012 \\
\hline & 35 & 9.399 & 9.398 & 9.400 & -0.008 & -0.019 & 0.002 \\
\hline & 40 & 9.399 & 9.398 & 9.400 & -0.008 & -0.017 & 0.002 \\
\hline & 45 & 9.400 & 9.399 & 9.401 & -0.001 & -0.010 & 0.008 \\
\hline & 50 & 9.400 & 9.399 & 9.401 & 0.001 & -0.008 & 0.009 \\
\hline & 100 & 9.399 & 9.399 & 9.400 & -0.008 & -0.015 & -0.002 \\
\hline sig_b3c & 5 & 1.024 & 1.005 & 1.042 & -14.693 & -16.245 & -13.142 \\
\hline \multirow[t]{10}{*}{$(1.2)$} & 10 & 1.124 & 1.112 & 1.135 & -6.355 & -7.333 & -5.378 \\
\hline & 15 & 1.157 & 1.148 & 1.166 & -3.576 & -4.334 & -2.819 \\
\hline & 20 & 1.168 & 1.161 & 1.176 & -2.645 & -3.276 & -2.014 \\
\hline & 25 & 1.177 & 1.170 & 1.184 & -1.918 & -2.473 & -1.363 \\
\hline & 30 & 1.183 & 1.176 & 1.189 & -1.457 & -1.966 & -0.947 \\
\hline & 35 & 1.182 & 1.177 & 1.188 & -1.465 & -1.924 & -1.006 \\
\hline & 40 & 1.186 & 1.181 & 1.191 & -1.152 & -1.580 & -0.723 \\
\hline & 45 & 1.187 & 1.183 & 1.192 & -1.055 & -1.458 & -0.653 \\
\hline & 50 & 1.189 & 1.184 & 1.193 & -0.949 & -1.330 & -0.568 \\
\hline & 100 & 1.197 & 1.194 & 1.200 & -0.265 & -0.532 & 0.002 \\
\hline sig_b4c & 5 & 1.093 & 1.078 & 1.107 & -14.693 & -16.245 & -13.142 \\
\hline \multirow[t]{10}{*}{$(1.2)$} & 10 & 1.156 & 1.147 & 1.164 & -6.355 & -7.333 & -5.378 \\
\hline & 15 & 1.174 & 1.167 & 1.181 & -3.576 & -4.334 & -2.819 \\
\hline & 20 & 1.176 & 1.170 & 1.181 & -2.645 & -3.276 & -2.014 \\
\hline & 25 & 1.189 & 1.184 & 1.195 & -1.918 & -2.473 & -1.363 \\
\hline & 30 & 1.191 & 1.187 & 1.196 & -1.457 & -1.966 & -0.947 \\
\hline & 35 & 1.189 & 1.185 & 1.193 & -1.465 & -1.924 & -1.006 \\
\hline & 40 & 1.191 & 1.187 & 1.195 & -1.152 & -1.580 & -0.723 \\
\hline & 45 & 1.192 & 1.188 & 1.196 & -1.055 & -1.458 & -0.653 \\
\hline & 50 & 1.193 & 1.190 & 1.197 & -0.949 & -1.330 & -0.568 \\
\hline & 100 & 1.198 & 1.195 & 1.200 & -0.265 & -0.532 & 0.002 \\
\hline
\end{tabular}

Continued overleaf 
Table A3 (continued). Hours, Extended Model: estimated parameters

\begin{tabular}{lccccrrr}
\hline Icc & 5 & 0.060 & 0.059 & 0.061 & -2.085 & -4.235 & 0.064 \\
$(0.061)$ & 10 & 0.060 & 0.059 & 0.061 & -1.476 & -2.809 & -0.144 \\
& 15 & 0.061 & 0.060 & 0.062 & -0.407 & -1.465 & 0.652 \\
& 20 & 0.061 & 0.060 & 0.061 & -0.475 & -1.374 & 0.424 \\
& 25 & 0.061 & 0.060 & 0.061 & -0.409 & -1.205 & 0.387 \\
& 30 & 0.061 & 0.060 & 0.061 & -0.501 & -1.238 & 0.236 \\
& 35 & 0.061 & 0.061 & 0.062 & -0.093 & -0.755 & 0.569 \\
& 40 & 0.061 & 0.061 & 0.062 & 0.090 & -0.537 & 0.717 \\
& 45 & 0.061 & 0.060 & 0.061 & -0.601 & -1.189 & -0.013 \\
& 50 & 0.061 & 0.061 & 0.062 & 0.072 & -0.486 & 0.631 \\
& 100 & 0.061 & 0.061 & 0.061 & -0.300 & -0.686 & 0.086 \\
\hline
\end{tabular}

Notes

(1) mean of distribution of parameter estimates from each Monte-Carlo replication

(2), (3): lower and upper bounds of 95\% CI for (1), calculated assuming normality of MC sampling distribution

(4) Relative bias: percentage difference between (1) and 'true' parameter value

(5), (6): lower and upper bounds of 95\% CI for (4), calculated assuming normality of MC sampling distribution 
Table A4. Hours, Extended Model: estimated standard errors and non-coverage rates

\begin{tabular}{|c|c|c|c|c|c|c|c|c|c|}
\hline Parameter & $N_{C}$ & $\begin{array}{c}\text { Empirical } \\
\text { SE } \\
(1)\end{array}$ & $\begin{array}{c}\text { Analytic } \\
\text { al SE } \\
(2) \\
\end{array}$ & LB & UB & $\begin{array}{c}\text { Relative } \\
\text { difference, } \\
\% \\
(5) \\
\end{array}$ & $\begin{array}{l}\text { Non- } \\
\text { coverage } \\
\text { rate, \% } \\
(6) \\
\end{array}$ & (7) & UB \\
\hline \multirow[t]{11}{*}{ cons } & 5 & 3.123 & 3.081 & 3.060 & 3.102 & -1.352 & 0.073 & 0.065 & 0.080 \\
\hline & 10 & 2.658 & 2.612 & 2.599 & 2.625 & -1.760 & 0.066 & 0.059 & 0.073 \\
\hline & 15 & 1.737 & 1.730 & 1.725 & 1.735 & -0.373 & 0.057 & 0.050 & 0.063 \\
\hline & 20 & 1.995 & 2.007 & 2.000 & 2.014 & 0.607 & 0.056 & 0.050 & 0.063 \\
\hline & 25 & 1.328 & 1.328 & 1.325 & 1.331 & -0.017 & 0.053 & 0.047 & 0.059 \\
\hline & 30 & 1.342 & 1.330 & 1.327 & 1.334 & -0.895 & 0.054 & 0.048 & 0.060 \\
\hline & 35 & 1.059 & 1.065 & 1.063 & 1.066 & 0.543 & 0.050 & 0.044 & 0.056 \\
\hline & 40 & 1.129 & 1.142 & 1.140 & 1.144 & 1.126 & 0.045 & 0.039 & 0.051 \\
\hline & 45 & 1.110 & 1.108 & 1.106 & 1.110 & -0.122 & 0.054 & 0.048 & 0.061 \\
\hline & 50 & 0.969 & 0.957 & 0.955 & 0.958 & -1.295 & 0.051 & 0.045 & 0.057 \\
\hline & 100 & 0.696 & 0.695 & 0.694 & 0.696 & -0.141 & 0.050 & 0.044 & 0.056 \\
\hline \multirow[t]{11}{*}{ age } & 5 & 0.102 & 0.100 & 0.100 & 0.100 & -2.164 & 0.058 & 0.051 & 0.064 \\
\hline & 10 & 0.071 & 0.071 & 0.071 & 0.071 & 0.110 & 0.050 & 0.044 & 0.056 \\
\hline & 15 & 0.058 & 0.058 & 0.058 & 0.058 & -0.270 & 0.052 & 0.046 & 0.058 \\
\hline & 20 & 0.050 & 0.050 & 0.050 & 0.050 & 0.027 & 0.050 & 0.044 & 0.056 \\
\hline & 25 & 0.045 & 0.045 & 0.045 & 0.045 & -0.450 & 0.052 & 0.046 & 0.058 \\
\hline & 30 & 0.041 & 0.041 & 0.041 & 0.041 & 0.812 & 0.046 & 0.040 & 0.052 \\
\hline & 35 & 0.038 & 0.038 & 0.038 & 0.038 & -0.285 & 0.050 & 0.044 & 0.056 \\
\hline & 40 & 0.035 & 0.036 & 0.036 & 0.036 & 1.888 & 0.045 & 0.039 & 0.051 \\
\hline & 45 & 0.034 & 0.034 & 0.034 & 0.034 & 0.005 & 0.049 & 0.043 & 0.055 \\
\hline & 50 & 0.032 & 0.032 & 0.032 & 0.032 & -0.657 & 0.053 & 0.046 & 0.059 \\
\hline & 100 & 0.023 & 0.023 & 0.023 & 0.023 & -0.475 & 0.051 & 0.045 & 0.057 \\
\hline \multirow[t]{11}{*}{ cohab } & 5 & 1.385 & 1.335 & 1.321 & 1.350 & -3.554 & 0.113 & 0.104 & 0.122 \\
\hline & 10 & 1.272 & 1.237 & 1.229 & 1.246 & -2.713 & 0.088 & 0.080 & 0.095 \\
\hline & 15 & 0.740 & 0.730 & 0.726 & 0.733 & -1.360 & 0.073 & 0.066 & 0.080 \\
\hline & 20 & 0.975 & 0.981 & 0.976 & 0.985 & 0.632 & 0.057 & 0.051 & 0.064 \\
\hline & 25 & 0.569 & 0.559 & 0.556 & 0.561 & -1.889 & 0.065 & 0.058 & 0.071 \\
\hline & 30 & 0.595 & 0.597 & 0.594 & 0.599 & 0.362 & 0.057 & 0.050 & 0.063 \\
\hline & 35 & 0.425 & 0.427 & 0.426 & 0.429 & 0.596 & 0.061 & 0.055 & 0.068 \\
\hline & 40 & 0.510 & 0.508 & 0.507 & 0.510 & -0.381 & 0.052 & 0.046 & 0.058 \\
\hline & 45 & 0.504 & 0.504 & 0.502 & 0.505 & -0.025 & 0.056 & 0.049 & 0.062 \\
\hline & 50 & 0.407 & 0.408 & 0.407 & 0.409 & 0.052 & 0.056 & 0.050 & 0.063 \\
\hline & 100 & 0.307 & 0.304 & 0.303 & 0.305 & -1.125 & 0.055 & 0.048 & 0.061 \\
\hline \multirow[t]{11}{*}{ nownch } & 5 & 1.255 & 1.163 & 1.149 & 1.176 & -7.397 & 0.140 & 0.130 & 0.149 \\
\hline & 10 & 1.128 & 1.100 & 1.092 & 1.107 & -2.552 & 0.087 & 0.079 & 0.095 \\
\hline & 15 & 0.663 & 0.648 & 0.645 & 0.652 & -2.171 & 0.072 & 0.065 & 0.079 \\
\hline & 20 & 0.887 & 0.868 & 0.864 & 0.872 & -2.073 & 0.066 & 0.059 & 0.073 \\
\hline & 25 & 0.501 & 0.498 & 0.496 & 0.500 & -0.561 & 0.061 & 0.054 & 0.068 \\
\hline & 30 & 0.547 & 0.531 & 0.529 & 0.533 & -2.797 & 0.066 & 0.059 & 0.073 \\
\hline & 35 & 0.380 & 0.380 & 0.378 & 0.381 & -0.143 & 0.057 & 0.051 & 0.064 \\
\hline & 40 & 0.447 & 0.451 & 0.450 & 0.452 & 0.937 & 0.057 & 0.050 & 0.063 \\
\hline & 45 & 0.446 & 0.447 & 0.446 & 0.448 & 0.253 & 0.059 & 0.052 & 0.065 \\
\hline & 50 & 0.368 & 0.363 & 0.362 & 0.364 & -1.454 & 0.056 & 0.049 & 0.062 \\
\hline & 100 & 0.273 & 0.270 & 0.270 & 0.271 & -0.892 & 0.054 & 0.048 & 0.060 \\
\hline
\end{tabular}

Continued overleaf 
Table A4 (contd.). Hours, Extended Model: estimated standard errors and noncoverage rates

\begin{tabular}{|c|c|c|c|c|c|c|c|c|c|}
\hline Parameter & $N_{C}$ & $\begin{array}{c}\text { Empirical } \\
\text { SE } \\
(1)\end{array}$ & $\begin{array}{l}\text { Analytic } \\
\text { al SE } \\
\text { (2) }\end{array}$ & LB & UB & $\begin{array}{c}\text { Relative } \\
\text { difference, } \\
\% \\
\text { (5) }\end{array}$ & $\begin{array}{l}\text { Non- } \\
\text { coverage } \\
\text { rate, \% } \\
\text { (6) }\end{array}$ & LB & UB \\
\hline \multirow[t]{11}{*}{ chexp } & 5 & 3.690 & 3.447 & 3.407 & 3.487 & -6.574 & 0.140 & 0.130 & 0.149 \\
\hline & 10 & 3.369 & 3.290 & 3.267 & 3.313 & -2.363 & 0.077 & 0.070 & 0.085 \\
\hline & 15 & 1.746 & 1.704 & 1.695 & 1.713 & -2.404 & 0.076 & 0.068 & 0.083 \\
\hline & 20 & 3.428 & 3.410 & 3.394 & 3.425 & -0.543 & 0.068 & 0.061 & 0.075 \\
\hline & 25 & 1.668 & 1.609 & 1.602 & 1.615 & -3.543 & 0.065 & 0.058 & 0.072 \\
\hline & 30 & 1.696 & 1.650 & 1.644 & 1.656 & -2.706 & 0.068 & 0.061 & 0.075 \\
\hline & 35 & 1.080 & 1.085 & 1.081 & 1.088 & 0.441 & 0.055 & 0.049 & 0.061 \\
\hline & 40 & 1.219 & 1.217 & 1.213 & 1.221 & -0.133 & 0.053 & 0.047 & 0.059 \\
\hline & 45 & 1.468 & 1.470 & 1.465 & 1.474 & 0.127 & 0.056 & 0.049 & 0.062 \\
\hline & 50 & 1.198 & 1.203 & 1.200 & 1.206 & 0.387 & 0.054 & 0.048 & 0.060 \\
\hline & 100 & 0.781 & 0.788 & 0.787 & 0.790 & 1.007 & 0.047 & 0.042 & 0.053 \\
\hline chexpX & 5 & 2.030 & 1.982 & 1.961 & 2.003 & -2.357 & 0.117 & 0.108 & 0.125 \\
\hline \multirow[t]{10}{*}{ cohab } & 10 & 1.911 & 1.848 & 1.835 & 1.860 & -3.301 & 0.084 & 0.076 & 0.092 \\
\hline & 15 & 0.982 & 0.957 & 0.952 & 0.963 & -2.516 & 0.077 & 0.070 & 0.084 \\
\hline & 20 & 1.925 & 1.921 & 1.912 & 1.930 & -0.203 & 0.060 & 0.053 & 0.066 \\
\hline & 25 & 0.930 & 0.907 & 0.904 & 0.911 & -2.426 & 0.065 & 0.059 & 0.072 \\
\hline & 30 & 0.940 & 0.933 & 0.929 & 0.936 & -0.733 & 0.060 & 0.053 & 0.066 \\
\hline & 35 & 0.611 & 0.611 & 0.609 & 0.613 & -0.013 & 0.058 & 0.051 & 0.064 \\
\hline & 40 & 0.687 & 0.687 & 0.685 & 0.689 & -0.008 & 0.053 & 0.047 & 0.059 \\
\hline & 45 & 0.829 & 0.832 & 0.829 & 0.834 & 0.349 & 0.052 & 0.046 & 0.059 \\
\hline & 50 & 0.671 & 0.676 & 0.675 & 0.678 & 0.818 & 0.051 & 0.045 & 0.058 \\
\hline & 100 & 0.450 & 0.446 & 0.445 & 0.447 & -0.995 & 0.055 & 0.049 & 0.062 \\
\hline chexpX & 5 & 1.881 & 1.730 & 1.709 & 1.750 & -8.044 & 0.144 & 0.134 & 0.153 \\
\hline \multirow[t]{10}{*}{ nownch } & 10 & 1.690 & 1.646 & 1.634 & 1.657 & -2.611 & 0.084 & 0.076 & 0.092 \\
\hline & 15 & 0.878 & 0.852 & 0.848 & 0.857 & -2.875 & 0.074 & 0.066 & 0.081 \\
\hline & 20 & 1.739 & 1.700 & 1.692 & 1.708 & -2.252 & 0.068 & 0.061 & 0.075 \\
\hline & 25 & 0.819 & 0.809 & 0.805 & 0.812 & -1.272 & 0.066 & 0.059 & 0.073 \\
\hline & 30 & 0.858 & 0.830 & 0.827 & 0.833 & -3.247 & 0.067 & 0.060 & 0.074 \\
\hline & 35 & 0.542 & 0.543 & 0.541 & 0.545 & 0.234 & 0.059 & 0.053 & 0.066 \\
\hline & 40 & 0.605 & 0.609 & 0.607 & 0.611 & 0.579 & 0.054 & 0.048 & 0.061 \\
\hline & 45 & 0.739 & 0.738 & 0.736 & 0.741 & -0.039 & 0.053 & 0.047 & 0.059 \\
\hline & 50 & 0.610 & 0.603 & 0.601 & 0.605 & -1.183 & 0.058 & 0.052 & 0.065 \\
\hline & 100 & 0.401 & 0.396 & 0.395 & 0.397 & -1.307 & 0.057 & 0.050 & 0.063 \\
\hline \multirow[t]{11}{*}{ sig_u } & 5 & 1.025 & 0.967 & 0.957 & 0.978 & -5.599 & 0.173 & 0.162 & 0.183 \\
\hline & 10 & 0.623 & 0.616 & 0.612 & 0.620 & -1.241 & 0.105 & 0.096 & 0.113 \\
\hline & 15 & 0.491 & 0.489 & 0.486 & 0.491 & -0.510 & 0.081 & 0.073 & 0.089 \\
\hline & 20 & 0.416 & 0.417 & 0.415 & 0.419 & 0.162 & 0.068 & 0.061 & 0.075 \\
\hline & 25 & 0.370 & 0.370 & 0.368 & 0.371 & -0.067 & 0.071 & 0.064 & 0.078 \\
\hline & 30 & 0.341 & 0.335 & 0.334 & 0.336 & -1.804 & 0.069 & 0.062 & 0.076 \\
\hline & 35 & 0.306 & 0.310 & 0.309 & 0.311 & 1.029 & 0.062 & 0.055 & 0.069 \\
\hline & 40 & 0.290 & 0.289 & 0.288 & 0.290 & -0.321 & 0.061 & 0.055 & 0.068 \\
\hline & 45 & 0.273 & 0.271 & 0.270 & 0.272 & -0.779 & 0.064 & 0.057 & 0.071 \\
\hline & 50 & 0.258 & 0.257 & 0.257 & 0.258 & -0.305 & 0.058 & 0.052 & 0.064 \\
\hline & 100 & 0.178 & 0.180 & 0.180 & 0.180 & 1.112 & 0.051 & 0.045 & 0.058 \\
\hline
\end{tabular}

Continued overleaf 
Table A4 (contd.). Hours, Extended Model: estimated standard errors and noncoverage rates

\begin{tabular}{|c|c|c|c|c|c|c|c|c|c|}
\hline Parameter & $N_{C}$ & $\begin{array}{c}\text { Empirical } \\
\text { SE }\end{array}$ & $\begin{array}{c}\text { Analytic } \\
\text { al SE } \\
(2)\end{array}$ & LB & UB & $\begin{array}{c}\text { Relative } \\
\text { difference, } \\
\% \\
(5) \\
\end{array}$ & $\begin{array}{l}\text { Non- } \\
\text { coverage } \\
\text { rate, \% } \\
(6)\end{array}$ & LB & UB \\
\hline \multirow[t]{11}{*}{ sig_e } & 5 & 0.095 & 0.094 & 0.094 & 0.095 & -1.127 & 0.060 & 0.053 & 0.066 \\
\hline & 10 & 0.067 & 0.067 & 0.067 & 0.067 & -0.216 & 0.049 & 0.043 & 0.055 \\
\hline & 15 & 0.055 & 0.054 & 0.054 & 0.054 & -0.875 & 0.052 & 0.046 & 0.058 \\
\hline & 20 & 0.047 & 0.047 & 0.047 & 0.047 & -0.594 & 0.051 & 0.045 & 0.058 \\
\hline & 25 & 0.042 & 0.042 & 0.042 & 0.042 & 0.326 & 0.050 & 0.044 & 0.056 \\
\hline & 30 & 0.038 & 0.038 & 0.038 & 0.038 & 0.777 & 0.048 & 0.042 & 0.054 \\
\hline & 35 & 0.035 & 0.036 & 0.036 & 0.036 & 0.560 & 0.051 & 0.045 & 0.057 \\
\hline & 40 & 0.033 & 0.033 & 0.033 & 0.033 & 0.720 & 0.044 & 0.039 & 0.050 \\
\hline & 45 & 0.031 & 0.031 & 0.031 & 0.031 & 1.698 & 0.044 & 0.039 & 0.050 \\
\hline & 50 & 0.029 & 0.030 & 0.030 & 0.030 & 2.348 & 0.047 & 0.041 & 0.052 \\
\hline & 100 & 0.021 & 0.021 & 0.021 & 0.021 & -1.084 & 0.053 & 0.047 & 0.060 \\
\hline \multirow[t]{11}{*}{ sig_b3c } & 5 & 0.668 & 0.609 & 0.601 & 0.618 & -8.747 & 0.151 & 0.141 & 0.160 \\
\hline & 10 & 0.422 & 0.402 & 0.400 & 0.405 & -4.738 & 0.036 & 0.031 & 0.041 \\
\hline & 15 & 0.328 & 0.316 & 0.315 & 0.317 & -3.541 & 0.067 & 0.060 & 0.074 \\
\hline & 20 & 0.273 & 0.269 & 0.268 & 0.270 & -1.513 & 0.065 & 0.058 & 0.071 \\
\hline & 25 & 0.240 & 0.237 & 0.237 & 0.238 & -1.200 & 0.059 & 0.052 & 0.066 \\
\hline & 30 & 0.221 & 0.216 & 0.215 & 0.216 & -2.215 & 0.063 & 0.056 & 0.070 \\
\hline & 35 & 0.199 & 0.198 & 0.198 & 0.199 & -0.279 & 0.053 & 0.047 & 0.060 \\
\hline & 40 & 0.185 & 0.185 & 0.185 & 0.185 & -0.218 & 0.057 & 0.051 & 0.064 \\
\hline & 45 & 0.174 & 0.174 & 0.173 & 0.174 & -0.405 & 0.054 & 0.048 & 0.060 \\
\hline & 50 & 0.165 & 0.164 & 0.164 & 0.165 & -0.425 & 0.054 & 0.048 & 0.061 \\
\hline & 100 & 0.116 & 0.115 & 0.115 & 0.115 & -0.708 & 0.052 & 0.045 & 0.058 \\
\hline \multirow[t]{11}{*}{ sig_b4c } & 5 & 0.515 & 0.492 & 0.487 & 0.498 & -4.478 & 0.169 & 0.159 & 0.180 \\
\hline & 10 & 0.316 & 0.311 & 0.309 & 0.313 & -1.654 & 0.109 & 0.100 & 0.117 \\
\hline & 15 & 0.253 & 0.247 & 0.245 & 0.248 & -2.314 & 0.091 & 0.083 & 0.099 \\
\hline & 20 & 0.209 & 0.210 & 0.209 & 0.211 & 0.553 & 0.076 & 0.068 & 0.083 \\
\hline & 25 & 0.190 & 0.187 & 0.187 & 0.188 & -1.425 & 0.069 & 0.062 & 0.076 \\
\hline & 30 & 0.171 & 0.170 & 0.169 & 0.171 & -0.453 & 0.063 & 0.056 & 0.070 \\
\hline & 35 & 0.156 & 0.156 & 0.156 & 0.157 & 0.050 & 0.065 & 0.058 & 0.071 \\
\hline & 40 & 0.147 & 0.146 & 0.145 & 0.146 & -0.794 & 0.062 & 0.055 & 0.069 \\
\hline & 45 & 0.137 & 0.137 & 0.137 & 0.137 & 0.308 & 0.062 & 0.055 & 0.069 \\
\hline & 50 & 0.133 & 0.130 & 0.130 & 0.130 & -2.425 & 0.069 & 0.062 & 0.076 \\
\hline & 100 & 0.093 & 0.091 & 0.091 & 0.091 & -1.537 & 0.055 & 0.049 & 0.062 \\
\hline
\end{tabular}

Notes

(1): Empirical SE: standard deviation of distribution of parameter estimates from each Monte-Carlo replication

(2): Analytical SE: mean of distribution of SE estimates from each Monte-Carlo replication

(3), (4): lower and upper bounds of 95\% CI for (1), calculated assuming normality of MC sampling distribution

(5): Relative difference: percentage difference between (2) and (1)

(6): Non-coverage rate: proportion of MC replications for which estimated 95\% CI did not contain the true parameter (CI calculated using fitted SEs).

(7), (8): lower and upper bounds of 95\% CI for (6), calculated assuming normality of MC sampling distribution 


\section{A.3 Participation: basic model $(R=5,000)$}

Logit(participation $)=-9.1+0.5 *$ age_ic $-0.006 *$ age-squared_ic $+0.02 *$ cohab_ic $-0.27 *$ nownch_ic $+0.7 *$ isced3_ic $+0.9 *$ isced4_ic $+1.4 *$ isced56_ic $+0.98 *$ chexp_c + u_c + e_ic

u_c $\sim \mathrm{N}\left(0,0.275^{\wedge} 2\right)$, e_ic $\sim \mathrm{N}\left(0,\left(\mathrm{pi}^{\wedge}{ }^{\wedge} / / 3\right)^{\wedge} 2\right), \quad$ cov $\left(\mathrm{u} \_\mathrm{c}, \mathrm{e} \_\mathrm{ic}\right)=0$ icc $=0.0224707$

Table A5. Participation, Basic Model: estimated parameters

\begin{tabular}{|c|c|c|c|c|c|c|c|}
\hline $\begin{array}{l}\text { Parameter } \\
\text { (true value) }\end{array}$ & $N_{C}$ & Mean & (2) & (3) & $\begin{array}{c}\text { Relative bias, \% } \\
\text { (mean) } \\
(4)\end{array}$ & (5) & UB \\
\hline cons & 5 & -9.120 & -9.133 & -9.108 & 0.221 & 0.084 & 0.358 \\
\hline \multirow[t]{10}{*}{$(-9.1)$} & 10 & -9.108 & -9.120 & -9.096 & 0.086 & -0.044 & 0.215 \\
\hline & 15 & -9.103 & -9.111 & -9.095 & 0.034 & -0.052 & 0.120 \\
\hline & 20 & -9.106 & -9.113 & -9.100 & 0.071 & -0.004 & 0.147 \\
\hline & 25 & -9.100 & -9.106 & -9.095 & 0.005 & -0.058 & 0.067 \\
\hline & 30 & -9.101 & -9.106 & -9.095 & 0.006 & -0.050 & 0.062 \\
\hline & 35 & -9.099 & -9.104 & -9.094 & -0.011 & -0.067 & 0.046 \\
\hline & 40 & -9.107 & -9.111 & -9.102 & 0.073 & 0.021 & 0.126 \\
\hline & 45 & -9.103 & -9.107 & -9.099 & 0.031 & -0.015 & 0.078 \\
\hline & 50 & -9.100 & -9.104 & -9.096 & -0.001 & -0.045 & 0.043 \\
\hline & 100 & -9.102 & -9.105 & -9.100 & 0.027 & -0.005 & 0.059 \\
\hline age & 5 & 0.501 & 0.501 & 0.502 & 0.233 & 0.112 & 0.354 \\
\hline \multirow[t]{10}{*}{$(0.5)$} & 10 & 0.501 & 0.500 & 0.501 & 0.127 & 0.042 & 0.212 \\
\hline & 15 & 0.500 & 0.500 & 0.501 & 0.045 & -0.025 & 0.115 \\
\hline & 20 & 0.500 & 0.500 & 0.500 & 0.036 & -0.024 & 0.095 \\
\hline & 25 & 0.500 & 0.500 & 0.500 & 0.019 & -0.034 & 0.072 \\
\hline & 30 & 0.500 & 0.500 & 0.500 & 0.028 & -0.022 & 0.078 \\
\hline & 35 & 0.500 & 0.500 & 0.500 & -0.001 & -0.048 & 0.045 \\
\hline & 40 & 0.500 & 0.500 & 0.500 & 0.050 & 0.008 & 0.092 \\
\hline & 45 & 0.500 & 0.500 & 0.500 & 0.034 & -0.006 & 0.074 \\
\hline & 50 & 0.500 & 0.500 & 0.500 & 0.001 & -0.037 & 0.039 \\
\hline & 100 & 0.500 & 0.500 & 0.500 & 0.012 & -0.015 & 0.039 \\
\hline cohab & 5 & 0.018 & 0.015 & 0.020 & -11.903 & -24.547 & 0.741 \\
\hline \multirow[t]{10}{*}{$(0.02)$} & 10 & 0.019 & 0.017 & 0.020 & -7.215 & -15.725 & 1.295 \\
\hline & 15 & 0.019 & 0.017 & 0.020 & -6.394 & -13.722 & 0.934 \\
\hline & 20 & 0.019 & 0.018 & 0.021 & -2.641 & -8.855 & 3.573 \\
\hline & 25 & 0.019 & 0.018 & 0.020 & -4.031 & -9.636 & 1.574 \\
\hline & 30 & 0.020 & 0.018 & 0.021 & -2.357 & -7.518 & 2.804 \\
\hline & 35 & 0.020 & 0.019 & 0.021 & 2.143 & -2.562 & 6.848 \\
\hline & 40 & 0.020 & 0.019 & 0.020 & -1.942 & -6.286 & 2.403 \\
\hline & 45 & 0.020 & 0.020 & 0.021 & 1.871 & -2.213 & 5.956 \\
\hline & 50 & 0.020 & 0.019 & 0.021 & -0.952 & -4.906 & 3.001 \\
\hline & 100 & 0.020 & 0.020 & 0.021 & 1.439 & -1.328 & 4.205 \\
\hline nownch & 5 & -0.270 & -0.271 & -0.269 & -0.008 & -0.466 & 0.451 \\
\hline \multirow[t]{10}{*}{$(-0.27)$} & 10 & -0.270 & -0.271 & -0.269 & -0.099 & -0.412 & 0.215 \\
\hline & 15 & -0.269 & -0.270 & -0.269 & -0.217 & -0.480 & 0.046 \\
\hline & 20 & -0.270 & -0.271 & -0.270 & 0.088 & -0.142 & 0.317 \\
\hline & 25 & -0.270 & -0.270 & -0.269 & -0.182 & -0.384 & 0.021 \\
\hline & 30 & -0.270 & -0.270 & -0.269 & -0.095 & -0.283 & 0.093 \\
\hline & 35 & -0.270 & -0.270 & -0.269 & -0.116 & -0.287 & 0.055 \\
\hline & 40 & -0.270 & -0.271 & -0.270 & 0.077 & -0.080 & 0.234 \\
\hline & 45 & -0.270 & -0.271 & -0.270 & 0.076 & -0.075 & 0.228 \\
\hline & 50 & -0.270 & -0.270 & -0.270 & -0.020 & -0.166 & 0.125 \\
\hline & 100 & -0.270 & -0.270 & -0.270 & -0.031 & -0.131 & 0.068 \\
\hline
\end{tabular}

Continued overleaf 
Table A5 (continued). Participation, Basic Model: estimated parameters

\begin{tabular}{|c|c|c|c|c|c|c|c|}
\hline $\begin{array}{l}\text { Parameter } \\
\text { (true value) }\end{array}$ & $N_{C}$ & Mean & (2) & (3) & $\begin{array}{c}\text { Relative bias, \% } \\
\text { (mean) } \\
(4)\end{array}$ & (5) & UB \\
\hline chexp & 5 & 0.980 & 0.971 & 0.988 & -0.044 & -0.909 & 0.820 \\
\hline \multirow[t]{10}{*}{ (0.98) } & 10 & 0.973 & 0.955 & 0.990 & -0.746 & -2.544 & 1.051 \\
\hline & 15 & 0.981 & 0.975 & 0.987 & 0.100 & -0.513 & 0.713 \\
\hline & 20 & 0.985 & 0.978 & 0.991 & 0.467 & -0.232 & 1.167 \\
\hline & 25 & 0.976 & 0.971 & 0.981 & -0.432 & -0.923 & 0.059 \\
\hline & 30 & 0.978 & 0.975 & 0.981 & -0.215 & -0.525 & 0.094 \\
\hline & 35 & 0.979 & 0.975 & 0.984 & -0.067 & -0.496 & 0.362 \\
\hline & 40 & 0.984 & 0.979 & 0.989 & 0.371 & -0.140 & 0.881 \\
\hline & 45 & 0.981 & 0.977 & 0.984 & 0.076 & -0.267 & 0.418 \\
\hline & 50 & 0.980 & 0.977 & 0.983 & 0.009 & -0.279 & 0.297 \\
\hline & 100 & 0.982 & 0.979 & 0.984 & 0.192 & -0.052 & 0.436 \\
\hline sig_u & 5 & 0.187 & 0.184 & 0.190 & -31.983 & -32.976 & -30.989 \\
\hline \multirow[t]{10}{*}{ (0.275) } & 10 & 0.233 & 0.231 & 0.235 & -15.380 & -16.058 & -14.703 \\
\hline & 15 & 0.249 & 0.247 & 0.250 & -9.624 & -10.175 & -9.073 \\
\hline & 20 & 0.257 & 0.256 & 0.258 & -6.523 & -6.997 & -6.049 \\
\hline & 25 & 0.259 & 0.258 & 0.260 & -5.857 & -6.284 & -5.430 \\
\hline & 30 & 0.262 & 0.261 & 0.263 & -4.728 & -5.116 & -4.341 \\
\hline & 35 & 0.264 & 0.263 & 0.265 & -4.056 & -4.417 & -3.695 \\
\hline & 40 & 0.265 & 0.264 & 0.266 & -3.506 & -3.838 & -3.174 \\
\hline & 45 & 0.266 & 0.266 & 0.267 & -3.115 & -3.433 & -2.796 \\
\hline & 50 & 0.268 & 0.267 & 0.268 & -2.706 & -3.010 & -2.402 \\
\hline & 100 & 0.272 & 0.271 & 0.272 & -1.271 & -1.486 & -1.056 \\
\hline icc & 5 & 0.013 & 0.013 & 0.014 & -41.018 & -42.501 & -39.535 \\
\hline \multirow[t]{10}{*}{$(0.022)$} & 10 & 0.017 & 0.017 & 0.018 & -22.449 & -23.623 & -21.275 \\
\hline & 15 & 0.019 & 0.019 & 0.019 & -14.391 & -15.392 & -13.390 \\
\hline & 20 & 0.020 & 0.020 & 0.020 & -9.735 & -10.617 & -8.853 \\
\hline & 25 & 0.020 & 0.020 & 0.021 & -9.004 & -9.801 & -8.207 \\
\hline & 30 & 0.021 & 0.021 & 0.021 & -7.287 & -8.017 & -6.556 \\
\hline & 35 & 0.021 & 0.021 & 0.021 & -6.261 & -6.947 & -5.575 \\
\hline & 40 & 0.021 & 0.021 & 0.021 & -5.461 & -6.095 & -4.828 \\
\hline & 45 & 0.021 & 0.021 & 0.022 & -4.818 & -5.426 & -4.210 \\
\hline & 50 & 0.022 & 0.021 & 0.022 & -4.149 & -4.733 & -3.566 \\
\hline & 100 & 0.022 & 0.022 & 0.022 & -1.934 & -2.350 & -1.518 \\
\hline
\end{tabular}

Notes

(1) mean of distribution of parameter estimates from each Monte-Carlo replication (2), (3): lower and upper bounds of 95\% CI for (1), calculated assuming normality of MC sampling distribution

(4) Relative bias: percentage difference between (1) and 'true' parameter value

(5), (6): lower and upper bounds of 95\% CI for (4), calculated assuming normality of MC sampling distribution 
Table A6. Participation, Basic Model: estimated standard errors and non-coverage rates

\begin{tabular}{|c|c|c|c|c|c|c|c|c|c|}
\hline Parameter & $N_{C}$ & $\begin{array}{c}\text { Empirical } \\
\text { SE } \\
(1)\end{array}$ & $\begin{array}{c}\text { Analytic } \\
\text { al SE } \\
(2)\end{array}$ & (3) & $(4)$ & $\begin{array}{c}\text { Relative } \\
\text { difference, } \\
\% \\
(5) \\
\end{array}$ & $\begin{array}{l}\text { Non- } \\
\text { coverage } \\
\text { rate, \% } \\
(6) \\
\end{array}$ & $(7)$ & (8) \\
\hline \multirow[t]{11}{*}{ cons } & 5 & 0.449 & 0.427 & 0.426 & 0.428 & -4.968 & 0.064 & 0.057 & 0.071 \\
\hline & 10 & 0.425 & 0.387 & 0.386 & 0.388 & -8.985 & 0.081 & 0.073 & 0.089 \\
\hline & 15 & 0.282 & 0.271 & 0.271 & 0.272 & -3.709 & 0.055 & 0.048 & 0.061 \\
\hline & 20 & 0.248 & 0.244 & 0.243 & 0.244 & -1.847 & 0.056 & 0.050 & 0.063 \\
\hline & 25 & 0.207 & 0.205 & 0.205 & 0.205 & -0.841 & 0.053 & 0.047 & 0.059 \\
\hline & 30 & 0.184 & 0.183 & 0.183 & 0.183 & -0.441 & 0.050 & 0.044 & 0.056 \\
\hline & 35 & 0.185 & 0.180 & 0.179 & 0.180 & -3.166 & 0.057 & 0.051 & 0.064 \\
\hline & 40 & 0.172 & 0.172 & 0.172 & 0.172 & -0.190 & 0.050 & 0.044 & 0.056 \\
\hline & 45 & 0.153 & 0.152 & 0.151 & 0.152 & -0.794 & 0.052 & 0.045 & 0.058 \\
\hline & 50 & 0.145 & 0.144 & 0.144 & 0.145 & -0.218 & 0.050 & 0.044 & 0.056 \\
\hline & 100 & 0.105 & 0.104 & 0.104 & 0.104 & -1.251 & 0.053 & 0.046 & 0.059 \\
\hline \multirow[t]{11}{*}{ age } & 5 & 0.022 & 0.022 & 0.022 & 0.022 & -0.706 & 0.051 & 0.045 & 0.057 \\
\hline & 10 & 0.015 & 0.015 & 0.015 & 0.015 & -1.717 & 0.058 & 0.051 & 0.064 \\
\hline & 15 & 0.013 & 0.013 & 0.013 & 0.013 & 0.288 & 0.049 & 0.043 & 0.055 \\
\hline & 20 & 0.011 & 0.011 & 0.011 & 0.011 & 0.991 & 0.049 & 0.043 & 0.055 \\
\hline & 25 & 0.010 & 0.010 & 0.010 & 0.010 & 1.344 & 0.047 & 0.041 & 0.053 \\
\hline & 30 & 0.009 & 0.009 & 0.009 & 0.009 & 0.373 & 0.049 & 0.043 & 0.055 \\
\hline & 35 & 0.008 & 0.008 & 0.008 & 0.008 & -1.411 & 0.051 & 0.045 & 0.057 \\
\hline & 40 & 0.008 & 0.008 & 0.008 & 0.008 & 0.104 & 0.051 & 0.045 & 0.058 \\
\hline & 45 & 0.007 & 0.007 & 0.007 & 0.007 & -0.331 & 0.047 & 0.041 & 0.053 \\
\hline & 50 & 0.007 & 0.007 & 0.007 & 0.007 & 0.070 & 0.053 & 0.047 & 0.059 \\
\hline & 100 & 0.005 & 0.005 & 0.005 & 0.005 & -0.691 & 0.052 & 0.046 & 0.058 \\
\hline \multirow[t]{11}{*}{ cohab } & 5 & 0.091 & 0.090 & 0.090 & 0.090 & -1.085 & 0.056 & 0.049 & 0.062 \\
\hline & 10 & 0.061 & 0.062 & 0.062 & 0.062 & 0.868 & 0.045 & 0.039 & 0.051 \\
\hline & 15 & 0.053 & 0.052 & 0.052 & 0.052 & -1.621 & 0.058 & 0.051 & 0.064 \\
\hline & 20 & 0.045 & 0.045 & 0.045 & 0.045 & -0.481 & 0.051 & 0.045 & 0.057 \\
\hline & 25 & 0.040 & 0.040 & 0.040 & 0.040 & -0.952 & 0.055 & 0.049 & 0.061 \\
\hline & 30 & 0.037 & 0.037 & 0.037 & 0.037 & -0.359 & 0.046 & 0.040 & 0.051 \\
\hline & 35 & 0.034 & 0.034 & 0.034 & 0.034 & 0.045 & 0.050 & 0.044 & 0.056 \\
\hline & 40 & 0.031 & 0.031 & 0.031 & 0.031 & -0.545 & 0.050 & 0.044 & 0.056 \\
\hline & 45 & 0.029 & 0.030 & 0.030 & 0.030 & 0.229 & 0.053 & 0.047 & 0.060 \\
\hline & 50 & 0.029 & 0.028 & 0.028 & 0.028 & -0.189 & 0.049 & 0.043 & 0.055 \\
\hline & 100 & 0.020 & 0.020 & 0.020 & 0.020 & -0.346 & 0.048 & 0.042 & 0.054 \\
\hline \multirow[t]{11}{*}{ nownch } & 5 & 0.045 & 0.045 & 0.044 & 0.045 & -0.295 & 0.048 & 0.042 & 0.054 \\
\hline & 10 & 0.031 & 0.030 & 0.030 & 0.030 & -0.500 & 0.049 & 0.043 & 0.055 \\
\hline & 15 & 0.026 & 0.026 & 0.026 & 0.026 & 1.219 & 0.048 & 0.042 & 0.054 \\
\hline & 20 & 0.022 & 0.022 & 0.022 & 0.022 & -1.543 & 0.051 & 0.045 & 0.057 \\
\hline & 25 & 0.020 & 0.020 & 0.020 & 0.020 & 0.107 & 0.050 & 0.044 & 0.056 \\
\hline & 30 & 0.018 & 0.018 & 0.018 & 0.018 & 0.365 & 0.047 & 0.041 & 0.053 \\
\hline & 35 & 0.017 & 0.017 & 0.017 & 0.017 & 0.223 & 0.050 & 0.044 & 0.056 \\
\hline & 40 & 0.015 & 0.015 & 0.015 & 0.015 & 0.578 & 0.048 & 0.042 & 0.054 \\
\hline & 45 & 0.015 & 0.015 & 0.015 & 0.015 & -1.508 & 0.053 & 0.047 & 0.059 \\
\hline & 50 & 0.014 & 0.014 & 0.014 & 0.014 & -1.034 & 0.054 & 0.048 & 0.060 \\
\hline & 100 & 0.010 & 0.010 & 0.010 & 0.010 & 1.005 & 0.044 & 0.038 & 0.049 \\
\hline
\end{tabular}

Continued overleaf 
Table A6 (continued). Participation, Basic Model: estimated standard errors and noncoverage rates

\begin{tabular}{|c|c|c|c|c|c|c|c|c|c|}
\hline Parameter & $N_{C}$ & $\begin{array}{c}\text { Empirical } \\
\text { SE } \\
\text { (1) }\end{array}$ & $\begin{array}{l}\text { Analytic } \\
\text { al SE } \\
(2)\end{array}$ & LB & UB & $\begin{array}{c}\text { Relative } \\
\text { difference, } \\
\% \\
(5)\end{array}$ & $\begin{array}{l}\text { Non- } \\
\text { coverage } \\
\text { rate, \% } \\
(6)\end{array}$ & LB & UB \\
\hline \multirow[t]{11}{*}{ chexp } & 5 & 0.306 & 0.226 & 0.223 & 0.228 & -26.133 & 0.198 & 0.187 & 0.209 \\
\hline & 10 & 0.636 & 0.542 & 0.538 & 0.546 & -14.745 & 0.124 & 0.114 & 0.133 \\
\hline & 15 & 0.217 & 0.197 & 0.196 & 0.198 & -9.244 & 0.091 & 0.083 & 0.099 \\
\hline & 20 & 0.247 & 0.230 & 0.229 & 0.231 & -6.862 & 0.082 & 0.074 & 0.089 \\
\hline & 25 & 0.174 & 0.165 & 0.164 & 0.165 & -5.106 & 0.073 & 0.065 & 0.080 \\
\hline & 30 & 0.109 & 0.105 & 0.104 & 0.105 & -4.437 & 0.071 & 0.064 & 0.078 \\
\hline & 35 & 0.152 & 0.145 & 0.145 & 0.146 & -4.141 & 0.065 & 0.058 & 0.072 \\
\hline & 40 & 0.181 & 0.179 & 0.178 & 0.180 & -0.863 & 0.059 & 0.053 & 0.066 \\
\hline & 45 & 0.121 & 0.120 & 0.120 & 0.120 & -0.892 & 0.057 & 0.051 & 0.063 \\
\hline & 50 & 0.102 & 0.100 & 0.100 & 0.101 & -1.541 & 0.061 & 0.054 & 0.068 \\
\hline & 100 & 0.086 & 0.086 & 0.085 & 0.086 & -0.891 & 0.054 & 0.048 & 0.061 \\
\hline \multirow[t]{11}{*}{ sig_u } & 5 & 0.099 & 0.077 & 0.076 & 0.078 & $\begin{array}{l}-21.721 \\
\end{array}$ & 0.375 & 0.361 & 0.388 \\
\hline & 10 & 0.067 & 0.059 & 0.059 & 0.059 & -12.319 & 0.219 & 0.208 & 0.230 \\
\hline & 15 & 0.055 & 0.051 & 0.050 & 0.051 & -7.307 & 0.159 & 0.149 & 0.169 \\
\hline & 20 & 0.047 & 0.045 & 0.045 & 0.045 & -4.420 & 0.121 & 0.112 & 0.130 \\
\hline & 25 & 0.042 & 0.040 & 0.040 & 0.041 & -4.646 & 0.119 & 0.110 & 0.128 \\
\hline & 30 & 0.038 & 0.037 & 0.037 & 0.038 & -2.686 & 0.104 & 0.095 & 0.112 \\
\hline & 35 & 0.036 & 0.035 & 0.035 & 0.035 & -3.073 & 0.096 & 0.088 & 0.104 \\
\hline & 40 & 0.033 & 0.033 & 0.032 & 0.033 & -1.248 & 0.091 & 0.083 & 0.099 \\
\hline & 45 & 0.032 & 0.031 & 0.031 & 0.031 & -2.654 & 0.088 & 0.080 & 0.095 \\
\hline & 50 & 0.030 & 0.029 & 0.029 & 0.029 & -2.528 & 0.082 & 0.074 & 0.089 \\
\hline & 100 & 0.021 & 0.021 & 0.021 & 0.021 & -1.571 & 0.068 & 0.061 & 0.075 \\
\hline \multirow[t]{11}{*}{ icc } & 5 & 0.012 & 0.009 & 0.009 & 0.010 & -22.322 & 0.457 & 0.443 & 0.471 \\
\hline & 10 & 0.010 & 0.008 & 0.008 & 0.009 & -11.065 & 0.284 & 0.272 & 0.297 \\
\hline & 15 & 0.008 & 0.008 & 0.008 & 0.008 & -6.331 & 0.204 & 0.192 & 0.215 \\
\hline & 20 & 0.007 & 0.007 & 0.007 & 0.007 & -3.687 & 0.159 & 0.149 & 0.169 \\
\hline & 25 & 0.006 & 0.006 & 0.006 & 0.006 & -3.880 & 0.146 & 0.137 & 0.156 \\
\hline & 30 & 0.006 & 0.006 & 0.006 & 0.006 & -2.134 & 0.133 & 0.124 & 0.143 \\
\hline & 35 & 0.006 & 0.005 & 0.005 & 0.005 & -2.919 & 0.118 & 0.109 & 0.127 \\
\hline & 40 & 0.005 & 0.005 & 0.005 & 0.005 & -1.182 & 0.109 & 0.100 & 0.117 \\
\hline & 45 & 0.005 & 0.005 & 0.005 & 0.005 & -2.224 & 0.105 & 0.096 & 0.113 \\
\hline & 50 & 0.005 & 0.005 & 0.005 & 0.005 & -2.375 & 0.098 & 0.090 & 0.106 \\
\hline & 100 & 0.003 & 0.003 & 0.003 & 0.003 & -1.383 & 0.076 & 0.068 & 0.083 \\
\hline
\end{tabular}

Notes

(1): Empirical SE: standard deviation of distribution of parameter estimates from each Monte-Carlo replication

(2): Analytical SE: mean of distribution of SE estimates from each Monte-Carlo replication

(3), (4): lower and upper bounds of 95\% CI for (1), calculated assuming normality of MC sampling distribution

(5): Relative difference: percentage difference between (2) and (1)

(6): Non-coverage rate: proportion of MC replications for which estimated 95\% CI did not contain the true parameter (CI calculated using fitted SEs).

(7), (8): lower and upper bounds of 95\% CI for (6), calculated assuming normality of MC sampling distribution 


\section{A.4 Participation, Extended Model $(R=1,000)$}

Logit $($ participation $)=-9.1+0.5 *$ age_ic $-0.006 *$ age-squared_ic $+(0.02+$ b3c $) *$ cohab_ic $-(0.27+b 4 c) *$ nownch_ic $+0.7 *$ isced3_ic $+0.9 *$ isced4_ic $+1.4 *$ isced56_ic $+0.7 *$ chexp_c $+0.6 *$ (chexp_c X cohab_ic) $-0.1 *$ (chexp_c X nownch_ic) + u_c + e_ic u_c $\sim \mathrm{N}(0,0.38 \wedge 2)$, e_ic $\sim \mathrm{N}\left(0,\left(\mathrm{pi}^{\wedge}{ }^{2} / 3\right)^{\wedge} 2\right), \quad$ cov$\left(\mathrm{u} \_\right.$c, e_ic $)=0$ icc $=0.0420468$ sig_b3c $=0.25$, sig_b4c $=0.13$

Table A7. Participation, Extended Model: estimated parameters

\begin{tabular}{|c|c|c|c|c|c|c|c|}
\hline $\begin{array}{l}\text { Parameter } \\
\text { (true value) }\end{array}$ & $N_{C}$ & (1) & $(2)$ & (3) & $\begin{array}{l}\text { Relative bias, } \\
\text { \% (mean) } \\
(4)\end{array}$ & (5) & (6) \\
\hline cons & 5 & -9.123 & -9.154 & -9.092 & 0.251 & -0.090 & 0.591 \\
\hline \multirow[t]{10}{*}{$(-9.1)$} & 10 & -9.121 & -9.154 & -9.088 & 0.229 & -0.136 & 0.594 \\
\hline & 15 & -9.095 & -9.116 & -9.075 & -0.050 & -0.271 & 0.172 \\
\hline & 20 & -9.103 & -9.122 & -9.084 & 0.034 & -0.171 & 0.239 \\
\hline & 25 & -9.104 & -9.119 & -9.089 & 0.046 & -0.118 & 0.210 \\
\hline & 30 & -9.107 & -9.120 & -9.094 & 0.076 & -0.063 & 0.215 \\
\hline & 35 & -9.111 & -9.124 & -9.098 & 0.116 & -0.027 & 0.259 \\
\hline & 40 & -9.110 & -9.123 & -9.098 & 0.114 & -0.025 & 0.253 \\
\hline & 45 & -9.108 & -9.119 & -9.097 & 0.086 & -0.035 & 0.207 \\
\hline & 50 & -9.102 & -9.113 & -9.091 & 0.023 & -0.096 & 0.142 \\
\hline & 100 & -9.093 & -9.101 & -9.085 & -0.078 & -0.163 & 0.007 \\
\hline age & 5 & 0.500 & 0.499 & 0.502 & 0.073 & -0.197 & 0.344 \\
\hline \multirow[t]{10}{*}{$(0.5)$} & 10 & 0.501 & 0.500 & 0.502 & 0.123 & -0.065 & 0.311 \\
\hline & 15 & 0.500 & 0.499 & 0.501 & 0.029 & -0.135 & 0.194 \\
\hline & 20 & 0.500 & 0.500 & 0.501 & 0.082 & -0.054 & 0.218 \\
\hline & 25 & 0.500 & 0.500 & 0.501 & 0.059 & -0.062 & 0.179 \\
\hline & 30 & 0.501 & 0.500 & 0.501 & 0.119 & 0.010 & 0.228 \\
\hline & 35 & 0.500 & 0.500 & 0.501 & 0.008 & -0.094 & 0.110 \\
\hline & 40 & 0.501 & 0.500 & 0.501 & 0.127 & 0.034 & 0.219 \\
\hline & 45 & 0.500 & 0.500 & 0.501 & 0.082 & -0.009 & 0.173 \\
\hline & 50 & 0.500 & 0.500 & 0.500 & -0.005 & -0.092 & 0.082 \\
\hline & 100 & 0.500 & 0.500 & 0.500 & -0.001 & -0.062 & 0.059 \\
\hline cohab & 5 & 0.023 & 0.007 & 0.039 & 17.346 & -62.683 & 97.375 \\
\hline \multirow[t]{10}{*}{$(0.02)$} & 10 & 0.003 & -0.019 & 0.024 & -85.267 & -192.878 & 22.345 \\
\hline & 15 & 0.013 & 0.002 & 0.025 & -34.069 & -90.803 & 22.665 \\
\hline & 20 & 0.017 & 0.007 & 0.028 & -12.500 & -65.711 & 40.710 \\
\hline & 25 & 0.021 & 0.013 & 0.029 & 6.628 & -33.061 & 46.317 \\
\hline & 30 & 0.018 & 0.012 & 0.025 & -8.579 & -40.080 & 22.922 \\
\hline & 35 & 0.018 & 0.010 & 0.025 & -12.385 & -48.637 & 23.866 \\
\hline & 40 & 0.025 & 0.017 & 0.032 & 23.028 & -14.476 & 60.533 \\
\hline & 45 & 0.017 & 0.011 & 0.023 & -14.908 & -45.281 & 15.464 \\
\hline & 50 & 0.022 & 0.017 & 0.028 & 12.279 & -16.730 & 41.288 \\
\hline & 100 & 0.019 & 0.014 & 0.023 & -7.037 & -27.730 & 13.656 \\
\hline
\end{tabular}

Continued overleaf 
Table A7 (continued). Participation, Extended Model: estimated parameters

\begin{tabular}{|c|c|c|c|c|c|c|c|}
\hline $\begin{array}{l}\text { Parameter } \\
\text { (true value) }\end{array}$ & $N_{C}$ & (1) & $(2)$ & (3) & $\begin{array}{l}\text { Relative bias, } \\
\text { \% (mean) } \\
(4) \\
\end{array}$ & (5) & (6) \\
\hline nownch & 5 & -0.271 & -0.279 & -0.263 & 0.324 & -2.753 & 3.402 \\
\hline \multirow[t]{10}{*}{$(-0.27)$} & 10 & -0.266 & -0.276 & -0.255 & -1.582 & -5.552 & 2.388 \\
\hline & 15 & -0.266 & -0.272 & -0.261 & -1.358 & -3.507 & 0.791 \\
\hline & 20 & -0.273 & -0.279 & -0.267 & 1.022 & -1.114 & 3.158 \\
\hline & 25 & -0.270 & -0.274 & -0.266 & 0.100 & -1.407 & 1.607 \\
\hline & 30 & -0.271 & -0.275 & -0.268 & 0.510 & -0.702 & 1.721 \\
\hline & 35 & -0.269 & -0.273 & -0.266 & -0.279 & -1.646 & 1.088 \\
\hline & 40 & -0.270 & -0.274 & -0.266 & -0.061 & -1.467 & 1.344 \\
\hline & 45 & -0.274 & -0.277 & -0.270 & 1.297 & 0.185 & 2.408 \\
\hline & 50 & -0.270 & -0.273 & -0.267 & 0.042 & -1.063 & 1.147 \\
\hline & 100 & -0.271 & -0.273 & -0.269 & 0.483 & -0.309 & 1.276 \\
\hline chexp & 5 & 0.720 & 0.694 & 0.747 & 2.916 & -0.895 & 6.728 \\
\hline \multirow[t]{10}{*}{$(0.7)$} & 10 & 0.726 & 0.671 & 0.781 & 3.747 & -4.133 & 11.627 \\
\hline & 15 & 0.692 & 0.673 & 0.710 & -1.191 & -3.872 & 1.489 \\
\hline & 20 & 0.701 & 0.679 & 0.722 & 0.076 & -2.943 & 3.094 \\
\hline & 25 & 0.699 & 0.684 & 0.714 & -0.191 & -2.345 & 1.963 \\
\hline & 30 & 0.701 & 0.691 & 0.710 & 0.100 & -1.277 & 1.476 \\
\hline & 35 & 0.711 & 0.698 & 0.724 & 1.563 & -0.277 & 3.404 \\
\hline & 40 & 0.700 & 0.684 & 0.716 & -0.060 & -2.341 & 2.221 \\
\hline & 45 & 0.701 & 0.690 & 0.713 & 0.210 & -1.439 & 1.859 \\
\hline & 50 & 0.697 & 0.685 & 0.708 & -0.476 & -2.114 & 1.161 \\
\hline & 100 & 0.690 & 0.682 & 0.699 & -1.360 & -2.533 & -0.187 \\
\hline chexpXcohab & 5 & 0.599 & 0.578 & 0.621 & -0.131 & -3.722 & 3.461 \\
\hline \multirow[t]{10}{*}{$(0.6)$} & 10 & 0.643 & 0.601 & 0.685 & 7.173 & 0.231 & 14.116 \\
\hline & 15 & 0.614 & 0.599 & 0.629 & 2.319 & -0.235 & 4.873 \\
\hline & 20 & 0.602 & 0.585 & 0.619 & 0.337 & -2.445 & 3.120 \\
\hline & 25 & 0.602 & 0.590 & 0.614 & 0.300 & -1.687 & 2.288 \\
\hline & 30 & 0.601 & 0.594 & 0.609 & 0.245 & -1.065 & 1.554 \\
\hline & 35 & 0.603 & 0.592 & 0.613 & 0.448 & -1.279 & 2.175 \\
\hline & 40 & 0.594 & 0.581 & 0.606 & -1.060 & -3.202 & 1.081 \\
\hline & 45 & 0.603 & 0.594 & 0.613 & 0.577 & -0.950 & 2.104 \\
\hline & 50 & 0.596 & 0.588 & 0.605 & -0.597 & -2.037 & 0.842 \\
\hline & 100 & 0.604 & 0.597 & 0.610 & 0.622 & -0.453 & 1.697 \\
\hline chexpXnownch & 5 & -0.097 & -0.108 & -0.086 & -2.724 & -13.826 & 8.377 \\
\hline \multirow[t]{10}{*}{$(-0.1)$} & 10 & -0.112 & -0.133 & -0.091 & 12.201 & -8.852 & 33.255 \\
\hline & 15 & -0.103 & -0.110 & -0.095 & 2.554 & -4.947 & 10.054 \\
\hline & 20 & -0.097 & -0.106 & -0.088 & -2.983 & -11.917 & 5.951 \\
\hline & 25 & -0.101 & -0.107 & -0.095 & 0.693 & -5.466 & 6.852 \\
\hline & 30 & -0.100 & -0.104 & -0.096 & 0.003 & -4.108 & 4.114 \\
\hline & 35 & -0.101 & -0.106 & -0.095 & 0.527 & -4.586 & 5.640 \\
\hline & 40 & -0.099 & -0.105 & -0.093 & -0.955 & -7.391 & 5.481 \\
\hline & 45 & -0.096 & -0.100 & -0.091 & -4.328 & -8.957 & 0.301 \\
\hline & 50 & -0.099 & -0.104 & -0.095 & -0.530 & -5.053 & 3.994 \\
\hline & 100 & -0.099 & -0.102 & -0.095 & -1.296 & -4.583 & 1.992 \\
\hline
\end{tabular}

Continued overleaf 
Table A7 (continued). Participation, Extended Model: estimated parameters

\begin{tabular}{|c|c|c|c|c|c|c|c|}
\hline $\begin{array}{l}\text { Parameter } \\
\text { (true value) }\end{array}$ & NC & Mean & LB & UB & $\begin{array}{r}\text { Relative bias, } \\
\% \text { (mean) } \\
\end{array}$ & LB & UB \\
\hline & & $(1)$ & $(2)$ & (3) & (4) & (5) & (6) \\
\hline sig_u & 5 & 0.249 & 0.240 & 0.258 & -34.412 & -36.768 & -32.056 \\
\hline \multirow[t]{10}{*}{$(0.38)$} & 10 & 0.329 & 0.323 & 0.336 & -13.291 & -14.895 & -11.688 \\
\hline & 15 & 0.344 & 0.339 & 0.349 & -9.369 & -10.667 & -8.072 \\
\hline & 20 & 0.356 & 0.352 & 0.360 & -6.354 & -7.452 & -5.256 \\
\hline & 25 & 0.357 & 0.354 & 0.361 & -5.945 & -6.919 & -4.972 \\
\hline & 30 & 0.364 & 0.361 & 0.368 & -4.206 & -5.128 & -3.285 \\
\hline & 35 & 0.364 & 0.361 & 0.368 & -4.100 & -4.931 & -3.269 \\
\hline & 40 & 0.366 & 0.363 & 0.369 & -3.593 & -4.361 & -2.826 \\
\hline & 45 & 0.369 & 0.366 & 0.372 & -2.931 & -3.635 & -2.227 \\
\hline & 50 & 0.372 & 0.369 & 0.374 & -2.165 & -2.881 & -1.448 \\
\hline & 100 & 0.375 & 0.373 & 0.377 & -1.359 & -1.848 & -0.869 \\
\hline sig_b3c & 5 & 0.129 & 0.121 & 0.136 & -48.589 & -51.639 & -45.540 \\
\hline \multirow[t]{10}{*}{$(0.25)$} & 10 & 0.194 & 0.188 & 0.200 & -22.291 & -24.656 & -19.926 \\
\hline & 15 & 0.215 & 0.211 & 0.220 & -13.845 & -15.728 & -11.962 \\
\hline & 20 & 0.223 & 0.219 & 0.227 & -10.863 & -12.524 & -9.203 \\
\hline & 25 & 0.228 & 0.225 & 0.232 & -8.649 & -10.060 & -7.238 \\
\hline & 30 & 0.235 & 0.232 & 0.238 & -6.100 & -7.363 & -4.836 \\
\hline & 35 & 0.234 & 0.232 & 0.237 & -6.249 & -7.391 & -5.107 \\
\hline & 40 & 0.240 & 0.238 & 0.243 & -3.852 & -4.925 & -2.778 \\
\hline & 45 & 0.239 & 0.236 & 0.241 & -4.579 & -5.605 & -3.553 \\
\hline & 50 & 0.241 & 0.239 & 0.244 & -3.519 & -4.460 & -2.579 \\
\hline & 100 & 0.245 & 0.243 & 0.247 & -1.981 & -2.650 & -1.312 \\
\hline sig_b4c & 5 & 0.074 & 0.070 & 0.078 & -43.315 & -46.451 & -40.179 \\
\hline \multirow[t]{10}{*}{$(0.13)$} & 10 & 0.102 & 0.100 & 0.105 & -21.195 & -23.432 & -18.959 \\
\hline & 15 & 0.113 & 0.110 & 0.115 & -13.387 & -15.162 & -11.613 \\
\hline & 20 & 0.116 & 0.114 & 0.118 & -10.688 & -12.222 & -9.153 \\
\hline & 25 & 0.119 & 0.118 & 0.121 & -8.112 & -9.469 & -6.755 \\
\hline & 30 & 0.122 & 0.120 & 0.123 & -6.440 & -7.650 & -5.231 \\
\hline & 35 & 0.122 & 0.121 & 0.124 & -5.874 & -6.926 & -4.823 \\
\hline & 40 & 0.123 & 0.122 & 0.124 & -5.427 & -6.420 & -4.435 \\
\hline & 45 & 0.126 & 0.124 & 0.127 & -3.456 & -4.391 & -2.522 \\
\hline & 50 & 0.125 & 0.124 & 0.126 & -3.898 & -4.810 & -2.987 \\
\hline & 100 & 0.127 & 0.127 & 0.128 & -2.042 & -2.681 & -1.404 \\
\hline icC & 5 & 0.024 & 0.023 & 0.025 & -42.905 & -46.294 & -39.516 \\
\hline \multirow[t]{10}{*}{$(0.042)$} & 10 & 0.034 & 0.033 & 0.035 & -18.394 & -21.144 & -15.643 \\
\hline & 15 & 0.036 & 0.035 & 0.037 & -13.588 & -15.868 & -11.308 \\
\hline & 20 & 0.038 & 0.037 & 0.039 & -9.282 & -11.300 & -7.264 \\
\hline & 25 & 0.038 & 0.037 & 0.039 & -9.087 & -10.870 & -7.304 \\
\hline & 30 & 0.039 & 0.039 & 0.040 & -6.127 & -7.841 & -4.412 \\
\hline & 35 & 0.039 & 0.039 & 0.040 & -6.264 & -7.814 & -4.714 \\
\hline & 40 & 0.040 & 0.039 & 0.040 & -5.542 & -6.980 & -4.104 \\
\hline & 45 & 0.040 & 0.040 & 0.041 & -4.506 & -5.826 & -3.186 \\
\hline & 50 & 0.041 & 0.040 & 0.041 & -3.037 & -4.388 & -1.686 \\
\hline & 100 & 0.041 & 0.041 & 0.042 & -2.089 & -3.020 & -1.159 \\
\hline
\end{tabular}

Notes

(1) mean of distribution of parameter estimates from each Monte-Carlo replication

(2), (3): lower and upper bounds of 95\% CI for (1), calculated assuming normality of MC sampling distribution

(4) Relative bias: percentage difference between (1) and 'true' parameter value

(5), (6): lower and upper bounds of 95\% CI for (4), calculated assuming normality of MC sampling distribution 
Table A8. Participation, Extended Model: estimated standard errors and non-coverage rates

\begin{tabular}{|c|c|c|c|c|c|c|c|c|c|}
\hline Parameter & $N_{C}$ & $\begin{array}{c}\text { Empirical } \\
\text { SE } \\
\text { (1) }\end{array}$ & $\begin{array}{c}\text { Analytical } \\
\text { SE } \\
\text { (2) }\end{array}$ & LB & UB & $\begin{array}{c}\text { Relative } \\
\text { difference, } \\
\% \\
(5)\end{array}$ & $\begin{array}{l}\text { Non- } \\
\text { coverage } \\
\text { rate, \% } \\
(6)\end{array}$ & LB & UB \\
\hline \multirow[t]{11}{*}{ cons } & 5 & 0.499 & 0.460 & 0.457 & 0.464 & -7.731 & 0.066 & 0.051 & 0.082 \\
\hline & 10 & 0.535 & 0.485 & 0.479 & 0.490 & -9.440 & 0.087 & 0.070 & 0.105 \\
\hline & 15 & 0.325 & 0.310 & 0.309 & 0.312 & -4.393 & 0.062 & 0.047 & 0.077 \\
\hline & 20 & 0.300 & 0.286 & 0.284 & 0.287 & -4.947 & 0.065 & 0.050 & 0.080 \\
\hline & 25 & 0.241 & 0.233 & 0.232 & 0.234 & -3.443 & 0.059 & 0.044 & 0.074 \\
\hline & 30 & 0.204 & 0.203 & 0.203 & 0.204 & -0.306 & 0.049 & 0.036 & 0.062 \\
\hline & 35 & 0.210 & 0.208 & 0.207 & 0.208 & -1.028 & 0.056 & 0.042 & 0.070 \\
\hline & 40 & 0.204 & 0.202 & 0.201 & 0.203 & -1.087 & 0.055 & 0.041 & 0.069 \\
\hline & 45 & 0.178 & 0.178 & 0.177 & 0.178 & -0.137 & 0.054 & 0.040 & 0.068 \\
\hline & 50 & 0.175 & 0.170 & 0.169 & 0.170 & -2.945 & 0.063 & 0.048 & 0.078 \\
\hline & 100 & 0.125 & 0.122 & 0.121 & 0.122 & -2.495 & 0.059 & 0.044 & 0.074 \\
\hline \multirow[t]{11}{*}{ age } & 5 & 0.022 & 0.022 & 0.022 & 0.022 & 0.271 & 0.038 & 0.026 & 0.050 \\
\hline & 10 & 0.015 & 0.015 & 0.015 & 0.015 & 0.432 & 0.055 & 0.041 & 0.069 \\
\hline & 15 & 0.013 & 0.013 & 0.013 & 0.013 & -3.626 & 0.060 & 0.045 & 0.075 \\
\hline & 20 & 0.011 & 0.011 & 0.011 & 0.011 & -0.462 & 0.044 & 0.031 & 0.057 \\
\hline & 25 & 0.010 & 0.010 & 0.010 & 0.010 & 0.062 & 0.041 & 0.029 & 0.053 \\
\hline & 30 & 0.009 & 0.009 & 0.009 & 0.009 & 3.745 & 0.035 & 0.024 & 0.046 \\
\hline & 35 & 0.008 & 0.008 & 0.008 & 0.008 & 1.069 & 0.049 & 0.036 & 0.062 \\
\hline & 40 & 0.007 & 0.008 & 0.008 & 0.008 & 2.618 & 0.047 & 0.034 & 0.060 \\
\hline & 45 & 0.007 & 0.007 & 0.007 & 0.007 & -1.278 & 0.053 & 0.039 & 0.067 \\
\hline & 50 & 0.007 & 0.007 & 0.007 & 0.007 & -1.712 & 0.058 & 0.044 & 0.072 \\
\hline & 100 & 0.005 & 0.005 & 0.005 & 0.005 & -0.046 & 0.041 & 0.029 & 0.053 \\
\hline \multirow[t]{11}{*}{ cohab } & 5 & 0.258 & 0.204 & 0.200 & 0.207 & -20.963 & 0.145 & 0.123 & 0.167 \\
\hline & 10 & 0.347 & 0.307 & 0.303 & 0.312 & -11.375 & 0.100 & 0.082 & 0.119 \\
\hline & 15 & 0.183 & 0.170 & 0.168 & 0.172 & -6.997 & 0.078 & 0.061 & 0.095 \\
\hline & 20 & 0.172 & 0.161 & 0.159 & 0.162 & -6.513 & 0.082 & 0.065 & 0.099 \\
\hline & 25 & 0.128 & 0.121 & 0.120 & 0.122 & -5.198 & 0.074 & 0.058 & 0.090 \\
\hline & 30 & 0.102 & 0.100 & 0.099 & 0.101 & -1.436 & 0.062 & 0.047 & 0.077 \\
\hline & 35 & 0.117 & 0.114 & 0.114 & 0.115 & -2.264 & 0.062 & 0.047 & 0.077 \\
\hline & 40 & 0.121 & 0.116 & 0.115 & 0.116 & -4.443 & 0.066 & 0.051 & 0.081 \\
\hline & 45 & 0.098 & 0.095 & 0.094 & 0.096 & -2.993 & 0.055 & 0.041 & 0.069 \\
\hline & 50 & 0.094 & 0.091 & 0.090 & 0.092 & -2.495 & 0.065 & 0.050 & 0.080 \\
\hline & 100 & 0.067 & 0.067 & 0.066 & 0.067 & -0.083 & 0.064 & 0.049 & 0.079 \\
\hline \multirow[t]{11}{*}{ nownch } & 5 & 0.134 & 0.105 & 0.102 & 0.107 & -21.825 & 0.166 & 0.143 & 0.189 \\
\hline & 10 & 0.173 & 0.155 & 0.153 & 0.157 & -10.239 & 0.101 & 0.083 & 0.120 \\
\hline & 15 & 0.094 & 0.086 & 0.085 & 0.087 & -8.083 & 0.085 & 0.068 & 0.102 \\
\hline & 20 & 0.093 & 0.082 & 0.081 & 0.083 & -12.172 & 0.098 & 0.080 & 0.116 \\
\hline & 25 & 0.066 & 0.062 & 0.061 & 0.062 & -5.679 & 0.074 & 0.058 & 0.090 \\
\hline & 30 & 0.053 & 0.051 & 0.051 & 0.052 & -2.998 & 0.071 & 0.055 & 0.087 \\
\hline & 35 & 0.060 & 0.058 & 0.058 & 0.058 & -2.506 & 0.069 & 0.053 & 0.085 \\
\hline & 40 & 0.061 & 0.058 & 0.058 & 0.058 & -5.171 & 0.066 & 0.051 & 0.081 \\
\hline & 45 & 0.048 & 0.049 & 0.048 & 0.049 & 0.393 & 0.053 & 0.039 & 0.067 \\
\hline & 50 & 0.048 & 0.046 & 0.046 & 0.047 & -3.824 & 0.059 & 0.044 & 0.074 \\
\hline & 100 & 0.035 & 0.034 & 0.034 & 0.034 & -1.792 & 0.044 & 0.031 & 0.057 \\
\hline
\end{tabular}

Continued overleaf 
Table A8 (continued). Participation, Extended Model: estimated standard errors and non-coverage rates

\begin{tabular}{|c|c|c|c|c|c|c|c|c|c|}
\hline Parameter & $N_{C}$ & $\begin{array}{c}\text { Empirical } \\
\text { SE }\end{array}$ & $\begin{array}{c}\text { Analytical } \\
\text { SE } \\
\text { (2) }\end{array}$ & LB & UB & $\begin{array}{c}\text { Relative } \\
\text { difference, } \\
\% \\
(5)\end{array}$ & $\begin{array}{l}\text { Non- } \\
\text { coverage } \\
\text { rate, \% } \\
\text { (6) }\end{array}$ & LB & UB \\
\hline \multirow[t]{11}{*}{ chexp } & 5 & 0.429 & 0.313 & 0.305 & 0.321 & -27.154 & 0.226 & 0.200 & 0.252 \\
\hline & 10 & 0.888 & 0.785 & 0.773 & 0.797 & -11.650 & 0.118 & 0.098 & 0.139 \\
\hline & 15 & 0.303 & 0.275 & 0.272 & 0.278 & -9.152 & 0.092 & 0.074 & 0.110 \\
\hline & 20 & 0.341 & 0.325 & 0.321 & 0.328 & -4.743 & 0.083 & 0.066 & 0.100 \\
\hline & 25 & 0.243 & 0.231 & 0.229 & 0.233 & -5.203 & 0.069 & 0.053 & 0.085 \\
\hline & 30 & 0.155 & 0.146 & 0.144 & 0.147 & -6.332 & 0.066 & 0.051 & 0.081 \\
\hline & 35 & 0.208 & 0.204 & 0.202 & 0.205 & -1.978 & 0.070 & 0.054 & 0.086 \\
\hline & 40 & 0.258 & 0.252 & 0.250 & 0.253 & -2.354 & 0.061 & 0.046 & 0.076 \\
\hline & 45 & 0.186 & 0.184 & 0.182 & 0.185 & -1.290 & 0.064 & 0.049 & 0.079 \\
\hline & 50 & 0.185 & 0.170 & 0.167 & 0.173 & -8.007 & 0.066 & 0.051 & 0.081 \\
\hline & 100 & 0.132 & 0.130 & 0.129 & 0.131 & -1.912 & 0.066 & 0.051 & 0.081 \\
\hline chexpX & 5 & 0.347 & 0.277 & 0.272 & 0.282 & -20.113 & 0.144 & 0.122 & 0.166 \\
\hline \multirow[t]{10}{*}{ Cohab } & 10 & 0.671 & 0.604 & 0.595 & 0.613 & -9.956 & 0.105 & 0.086 & 0.125 \\
\hline & 15 & 0.247 & 0.228 & 0.226 & 0.231 & -7.648 & 0.077 & 0.060 & 0.094 \\
\hline & 20 & 0.269 & 0.251 & 0.249 & 0.254 & -6.639 & 0.086 & 0.069 & 0.103 \\
\hline & 25 & 0.192 & 0.184 & 0.182 & 0.185 & -4.514 & 0.068 & 0.052 & 0.084 \\
\hline & 30 & 0.127 & 0.123 & 0.122 & 0.124 & -2.746 & 0.066 & 0.051 & 0.081 \\
\hline & 35 & 0.167 & 0.161 & 0.160 & 0.163 & -3.445 & 0.053 & 0.039 & 0.067 \\
\hline & 40 & 0.207 & 0.197 & 0.196 & 0.198 & -4.945 & 0.070 & 0.054 & 0.086 \\
\hline & 45 & 0.148 & 0.145 & 0.144 & 0.146 & -1.841 & 0.054 & 0.040 & 0.068 \\
\hline & 50 & 0.139 & 0.135 & 0.133 & 0.137 & -2.977 & 0.053 & 0.039 & 0.067 \\
\hline & 100 & 0.104 & 0.102 & 0.102 & 0.103 & -1.559 & 0.053 & 0.039 & 0.067 \\
\hline chexpX & 5 & 0.179 & 0.142 & 0.140 & 0.145 & -20.298 & 0.156 & 0.133 & 0.178 \\
\hline \multirow[t]{10}{*}{ nownch } & 10 & 0.339 & 0.305 & 0.300 & 0.309 & -10.078 & 0.104 & 0.085 & 0.123 \\
\hline & 15 & 0.121 & 0.115 & 0.113 & 0.116 & -5.254 & 0.068 & 0.052 & 0.084 \\
\hline & 20 & 0.144 & 0.129 & 0.127 & 0.130 & -10.807 & 0.098 & 0.080 & 0.116 \\
\hline & 25 & 0.099 & 0.094 & 0.093 & 0.095 & -5.196 & 0.076 & 0.060 & 0.092 \\
\hline & 30 & 0.066 & 0.064 & 0.063 & 0.064 & -4.159 & 0.074 & 0.058 & 0.090 \\
\hline & 35 & 0.082 & 0.082 & 0.082 & 0.083 & -0.394 & 0.058 & 0.044 & 0.072 \\
\hline & 40 & 0.104 & 0.099 & 0.098 & 0.100 & -4.628 & 0.062 & 0.047 & 0.077 \\
\hline & 45 & 0.075 & 0.074 & 0.074 & 0.075 & -0.584 & 0.057 & 0.043 & 0.071 \\
\hline & 50 & 0.073 & 0.069 & 0.068 & 0.070 & -5.970 & 0.065 & 0.050 & 0.080 \\
\hline & 100 & 0.053 & 0.052 & 0.052 & 0.053 & -1.646 & 0.066 & 0.051 & 0.081 \\
\hline \multirow[t]{11}{*}{ sig_u } & 5 & 0.144 & 0.108 & 0.106 & 0.110 & -24.934 & 0.390 & 0.360 & 0.420 \\
\hline & 10 & 0.098 & 0.085 & 0.084 & 0.086 & -13.293 & 0.198 & 0.173 & 0.223 \\
\hline & 15 & 0.080 & 0.072 & 0.071 & 0.072 & -9.849 & 0.154 & 0.132 & 0.176 \\
\hline & 20 & 0.067 & 0.063 & 0.063 & 0.064 & -5.874 & 0.124 & 0.104 & 0.144 \\
\hline & 25 & 0.060 & 0.057 & 0.056 & 0.057 & -4.806 & 0.118 & 0.098 & 0.138 \\
\hline & 30 & 0.056 & 0.053 & 0.052 & 0.053 & -6.520 & 0.107 & 0.088 & 0.126 \\
\hline & 35 & 0.051 & 0.049 & 0.049 & 0.049 & -4.099 & 0.089 & 0.071 & 0.107 \\
\hline & 40 & 0.047 & 0.046 & 0.046 & 0.046 & -2.579 & 0.084 & 0.067 & 0.101 \\
\hline & 45 & 0.043 & 0.043 & 0.043 & 0.044 & 0.647 & 0.071 & 0.055 & 0.087 \\
\hline & 50 & 0.044 & 0.042 & 0.041 & 0.042 & -5.456 & 0.092 & 0.074 & 0.110 \\
\hline & 100 & 0.030 & 0.030 & 0.029 & 0.030 & -1.655 & 0.063 & 0.048 & 0.078 \\
\hline
\end{tabular}

Continued overleaf 
Table A8 (continued). Participation, Extended Model: estimated standard errors and non-coverage rates

\begin{tabular}{|c|c|c|c|c|c|c|c|c|c|}
\hline Parameter & $N_{C}$ & $\begin{array}{c}\text { Empirical } \\
\text { SE }\end{array}$ & $\begin{array}{c}\text { Analytical } \\
\text { SE } \\
(2) \\
\end{array}$ & LB & UB & $\begin{array}{c}\text { Relative } \\
\text { difference, } \\
\% \\
(5) \\
\end{array}$ & $\begin{array}{c}\text { Non- } \\
\text { coverage } \\
\text { rate, \% } \\
(6)\end{array}$ & LB & UB \\
\hline \multirow[t]{11}{*}{ sig_b3c } & 5 & 0.123 & 0.133 & 0.128 & 0.137 & 8.287 & 0.231 & 0.205 & 0.257 \\
\hline & 10 & 0.095 & 0.090 & 0.088 & 0.093 & -5.233 & 0.050 & 0.037 & 0.064 \\
\hline & 15 & 0.076 & 0.072 & 0.070 & 0.073 & -5.656 & 0.049 & 0.036 & 0.062 \\
\hline & 20 & 0.067 & 0.060 & 0.060 & 0.061 & -10.047 & 0.081 & 0.064 & 0.098 \\
\hline & 25 & 0.057 & 0.053 & 0.053 & 0.054 & -6.158 & 0.086 & 0.069 & 0.103 \\
\hline & 30 & 0.051 & 0.049 & 0.049 & 0.049 & -3.890 & 0.068 & 0.052 & 0.084 \\
\hline & 35 & 0.046 & 0.045 & 0.045 & 0.045 & -2.778 & 0.083 & 0.066 & 0.100 \\
\hline & 40 & 0.043 & 0.042 & 0.041 & 0.042 & -4.000 & 0.070 & 0.054 & 0.086 \\
\hline & 45 & 0.041 & 0.039 & 0.039 & 0.039 & -5.299 & 0.083 & 0.066 & 0.100 \\
\hline & 50 & 0.038 & 0.037 & 0.037 & 0.038 & -1.407 & 0.067 & 0.051 & 0.083 \\
\hline & 100 & 0.027 & 0.026 & 0.026 & 0.026 & -2.364 & 0.064 & 0.049 & 0.079 \\
\hline \multirow[t]{11}{*}{ sig_b4c } & 5 & 0.066 & 0.070 & 0.067 & 0.073 & 6.843 & 0.192 & 0.167 & 0.216 \\
\hline & 10 & 0.047 & 0.044 & 0.043 & 0.045 & -5.468 & 0.056 & 0.042 & 0.071 \\
\hline & 15 & 0.037 & 0.036 & 0.035 & 0.036 & -4.441 & 0.079 & 0.062 & 0.096 \\
\hline & 20 & 0.032 & 0.030 & 0.029 & 0.031 & -6.362 & 0.109 & 0.090 & 0.128 \\
\hline & 25 & 0.028 & 0.027 & 0.026 & 0.027 & -6.687 & 0.093 & 0.075 & 0.111 \\
\hline & 30 & 0.025 & 0.024 & 0.024 & 0.025 & -3.683 & 0.070 & 0.054 & 0.086 \\
\hline & 35 & 0.022 & 0.022 & 0.022 & 0.022 & 1.054 & 0.069 & 0.053 & 0.085 \\
\hline & 40 & 0.021 & 0.021 & 0.021 & 0.021 & -0.760 & 0.072 & 0.056 & 0.088 \\
\hline & 45 & 0.020 & 0.020 & 0.020 & 0.020 & 0.129 & 0.062 & 0.047 & 0.077 \\
\hline & 50 & 0.019 & 0.019 & 0.019 & 0.019 & -2.060 & 0.075 & 0.059 & 0.091 \\
\hline & 100 & 0.013 & 0.013 & 0.013 & 0.013 & -1.381 & 0.062 & 0.047 & 0.077 \\
\hline \multirow[t]{11}{*}{ icchat } & 5 & 0.023 & 0.017 & 0.016 & 0.018 & -26.398 & 0.468 & 0.437 & 0.499 \\
\hline & 10 & 0.019 & 0.017 & 0.016 & 0.017 & -11.112 & 0.250 & 0.223 & 0.277 \\
\hline & 15 & 0.015 & 0.014 & 0.014 & 0.015 & -7.591 & 0.202 & 0.177 & 0.227 \\
\hline & 20 & 0.014 & 0.013 & 0.013 & 0.013 & -5.616 & 0.156 & 0.133 & 0.179 \\
\hline & 25 & 0.012 & 0.012 & 0.011 & 0.012 & -4.185 & 0.141 & 0.119 & 0.163 \\
\hline & 30 & 0.012 & 0.011 & 0.011 & 0.011 & -6.176 & 0.137 & 0.116 & 0.158 \\
\hline & 35 & 0.011 & 0.010 & 0.010 & 0.010 & -4.038 & 0.107 & 0.088 & 0.126 \\
\hline & 40 & 0.010 & 0.009 & 0.009 & 0.010 & -2.686 & 0.100 & 0.081 & 0.119 \\
\hline & 45 & 0.009 & 0.009 & 0.009 & 0.009 & 0.930 & 0.081 & 0.064 & 0.098 \\
\hline & 50 & 0.009 & 0.009 & 0.009 & 0.009 & -5.118 & 0.107 & 0.088 & 0.126 \\
\hline & 100 & 0.006 & 0.006 & 0.006 & 0.006 & -1.719 & 0.074 & 0.058 & 0.090 \\
\hline
\end{tabular}

Notes

(1): Empirical SE: standard deviation of distribution of parameter estimates from each Monte-Carlo replication

(2): Analytical SE: mean of distribution of SE estimates from each Monte-Carlo replication

(3), (4): lower and upper bounds of 95\% CI for (1), calculated assuming normality of MC sampling distribution

(5): Relative difference: percentage difference between (2) and (1)

(6): Non-coverage rate: proportion of MC replications for which estimated 95\% CI did not contain the true parameter (CI calculated using fitted SEs).

(7), (8): lower and upper bounds of 95\% CI for (6), calculated assuming normality of MC sampling distribution 